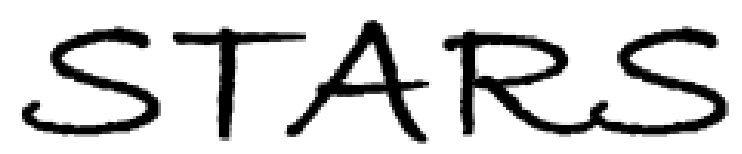

University of Central Florida

STARS

2019

\title{
Sub-Terahertz Spin Pumping from an Insulating Antiferromagnet
}

Priyanka Vaidya

University of Central Florida

\section{Part of the Physics Commons}

Find similar works at: https://stars.library.ucf.edu/etd

University of Central Florida Libraries http://library.ucf.edu

This Doctoral Dissertation (Open Access) is brought to you for free and open access by STARS. It has been accepted for inclusion in Electronic Theses and Dissertations by an authorized administrator of STARS. For more information, please contact STARS@ucf.edu.

\section{STARS Citation}

Vaidya, Priyanka, "Sub-Terahertz Spin Pumping from an Insulating Antiferromagnet" (2019). Electronic Theses and Dissertations. 6757.

https://stars.library.ucf.edu/etd/6757

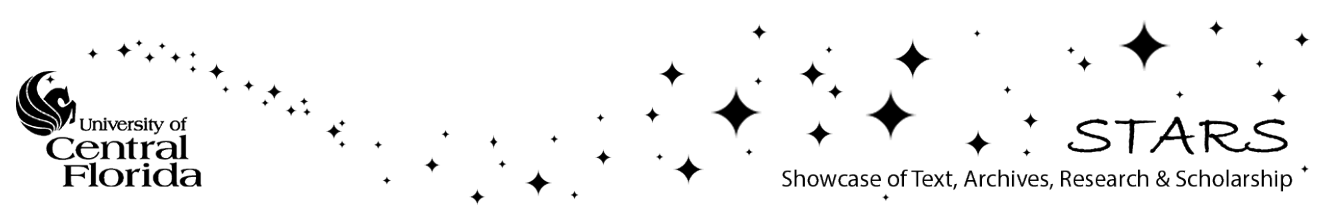




\title{
SUB-TERAHERTZ SPIN PUMPING \\ FROM AN INSULATING ANTIFERROMAGNET
}

by

\author{
PRIYANKA VAIDYA \\ B.Sc. Tribhuwan University, 2008 \\ M.Sc. Tribhuwan University, 2012 \\ M.S. University of Central Florida, 2016
}
A dissertation submitted in partial fulfillment of the requirements for the degree of Doctor of Philosophy in the Department of Physics in the College of Sciences at the University of Central Florida
Orlando, Florida

\section{Fall Term}

2019

Major Professor: Enrique del Barco 
(C) 2019 Priyanka Vaidya 


\begin{abstract}
The combination of the spin transfer torque and spin Hall effects, or their reciprocal dynamical spin pumping and inverse spin Hall effects, respectively, enable reading and controlling the magnetization state in spintronics devices which are at the verge of mass commercialization as the next generation of energy-efficient and fast magnetic random-access memory applications with the use of ferromagnetic elements, e.g., the spin valve. However, these effects have remained elusive in antiferromagnetic-based devices up to date, despite the fascinating advantages offered by the absence of stray fields (zero net magnetization), Terahertz spin dynamics, and the widespread availability of metallic, insulating and semiconducting antiferromagnetic materials. In this thesis I report the first demonstration of sub-Terahertz dynamical spin pumping at the interface between an antiferromagnet and a non-magnetic material; more specifically a uniaxial insulating antiferromagnet $\mathrm{MnF}_{2}$ and heavy metal Pt. The measured ISHE signal generated by the corresponding spin-charge current interconversion in the platinum layer is modulated by the handedness of the circularly polarized sub-THz irradiation. This effect results directly from the opposite chirality of each of the fundamental dynamical modes of the antiferromagnet. Contrary to the case of ferromagnets, this observation in an antiferromagnetic system allows unambiguously differentiating coherent spin pumping from incoherent spin Seeback effect, by which electric signals result from thermal activation. A complete study of the generated electric signals at the antiferromagnetic resonances, the spin-flop mode and the transition between the two regimes as the microwave polarization is continuously varied from circular to linear polarizations enabled an understanding of the different phenomena governing interconversion of spin dynamics and charge currents at the $\mathrm{MnF}_{2} / \mathrm{Pt}$ interface.
\end{abstract}


To my parents and my husband,

for their love, support and blessings 


\section{ACKNOWLEDGEMENTS}

I would like to take this opportunity to show my gratitude to those without whose love, support and guidance, my $\mathrm{PhD}$ would not be a successful one.

I would like to thank my $\mathrm{PhD}$ advisor Dr. Enrique del Barco who accepted me into his group and showed me the proper way to do research. He introduced me to an exciting and growing field of research and provided me various opportunities to travel to workshops and conferences. His teachings, advise and suggestions will always stay with me as I find my way in my career after $\mathrm{PhD}$.

I would also like to thank my colleagues in my research group, past and present, who have always stood shoulder to shoulder with me as we found our way through the maze of equipment failures, bureaucratic jungle and stressful deadlines. I will forever be grateful to my senior colleagues Marta, Alvar and Jim who not only trained me on all the lab skills I acquired during my PhD but also advised me on my projects at every step. I want to thank Rebecca whose friendly advice in and out of the lab have helped me a lot through the years. I want to thank Cameron, the analyst in our group, for not only helping me when I got stuck but also for lending me an ear when I needed it. In addition to being such a great friend that I can always count on, you have always put up with my shenanigans. Rebecca and Cameron have helped me immensely when my simulation work was going nowhere and provided me valuable insight on what I could try next. I also want to thank Gyan for being a source of positive reinforcement and for providing an atmosphere of hard work 
in the group. I am quite sure you will continue working on our project and take it to new heights, with new discoveries and very impactful papers.I want to wish the future generation of graduate students in our group whom I have come to know - Justin and Francis, many fruitful years of graduate studies and even more fruitful careers thereafter. I also want to show my pleasure in knowing our post-docs - Ran and Jaesuk and our visiting scientist Yan from whom I am hoping to learn so much more.

I cannot forget my friends in the department outside my group. I want to thank Tommy for always being there for inspiring and fruitful discussions. I will always cherish the talks, walks and lunches during which we shared so much! I would like to remember my friends from the beginning of my PhD - Isaac, Shima, Bijoya, Asim, Nabin, Naseem and others. I want to thank Seth and Masa for making our office talks very interesting. I also want to thank my Nepalese student community and the Orlando resident Nepalese community in general for providing that familiar atmosphere in an unfamiliar land.

In the end I want to thank my family - my parents and my husband. Coming from a traditionally patriarchal society I have faced many challenges, but my parents have always stood by my side, allowing me to fly high and wide in my ambitions. I have found the most loving, caring and supportive life partner in my husband Sabin who not only pampers me but also inspires me to try my best. Without these people, my achievements and my life would be incomplete. 


\section{TABLE OF CONTENTS}

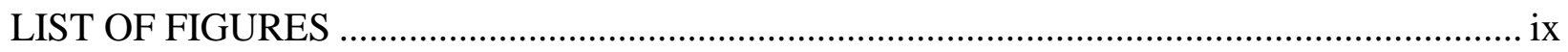

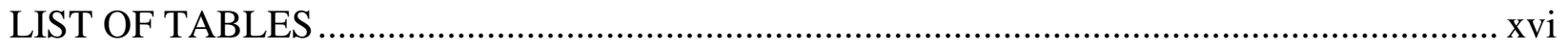

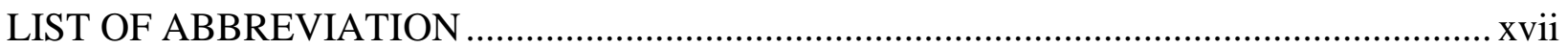

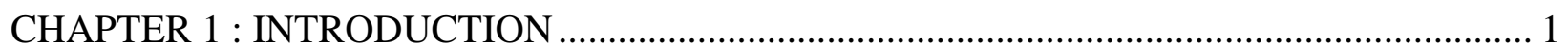

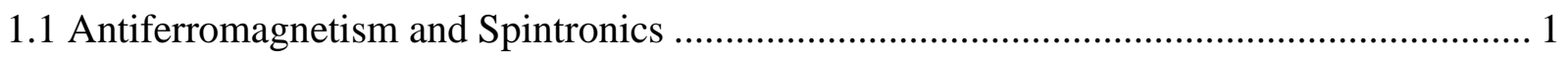

1.2 Antiferromagnetic Materials ............................................................................................ 3

1.3 Control and Manipulation of Antiferromagnetic Order ..................................................... 4

1.3.1 Manipulation by Magnetic Field .......................................................................... 4

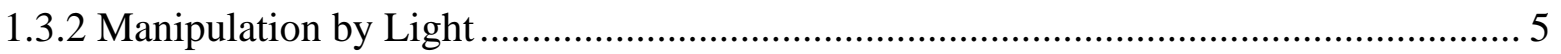

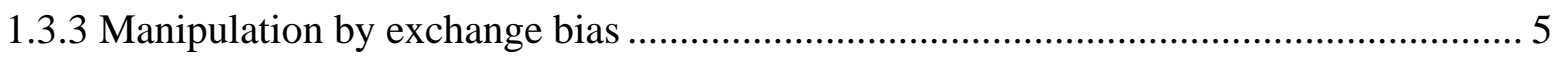

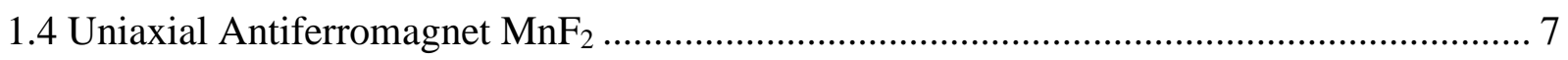

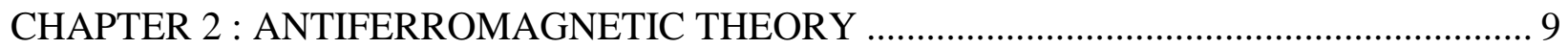

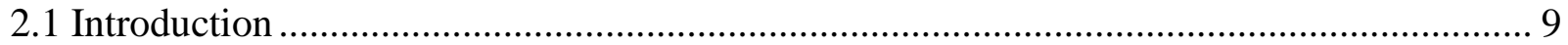

2.2 Magnetic Properties............................................................................................... 9

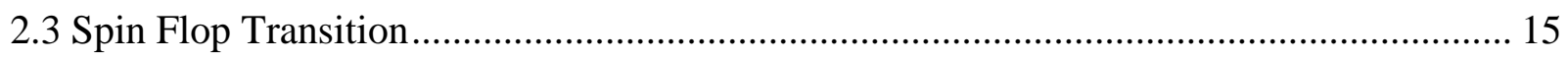

2.4 Energy contributions in a uniaxial Antiferromagnet..................................................... 16

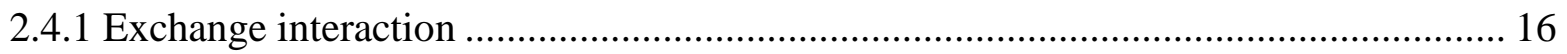

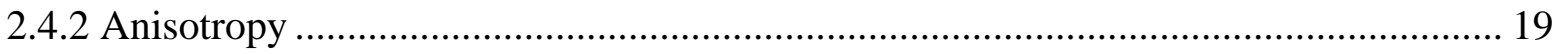

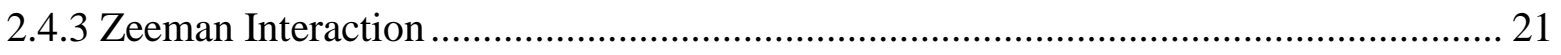

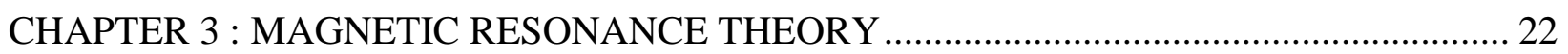

3.1 Electron Paramagnetic Resonance ................................................................................ 22

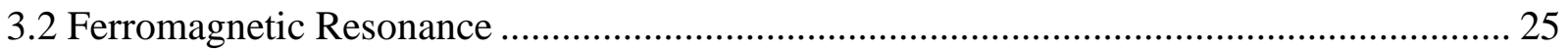

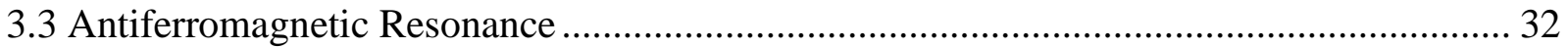

3.3.1 Spin-Flop: Critical Field Resonance .................................................................... 38

3.4 Phenomenological approach to Antiferromagnetic Resonance ………………….............. 40

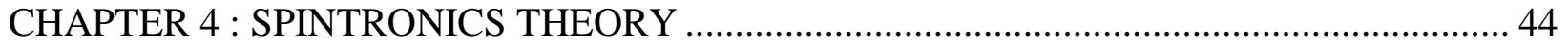

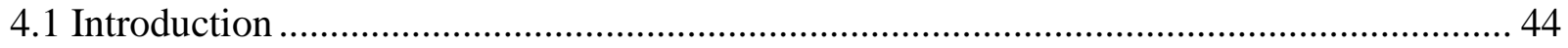


4.2 Spin Hall Effect and Inverse Spin Hall Effect .................................................................. 46

4.3 Spin Pumping and Spin Transfer Torque..................................................................... 47

4.3.1 Spin Pumping in a Ferromagnet ……......................................................................... 48

4.3.2 Spin Pumping in Antiferromagnets ......................................................................... 50

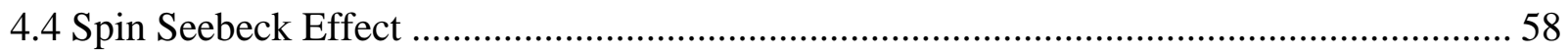

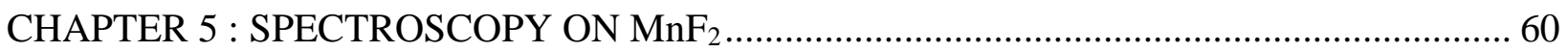

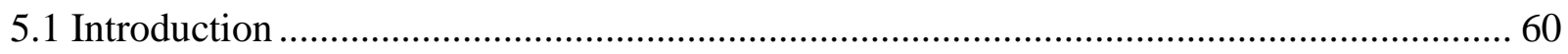

5.2 Experimental Setup: Quasi-heterodyne Spectrometer …………….................................... 62

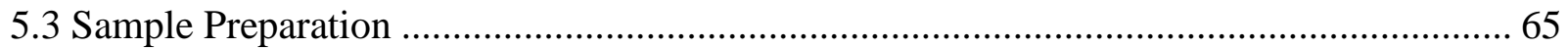

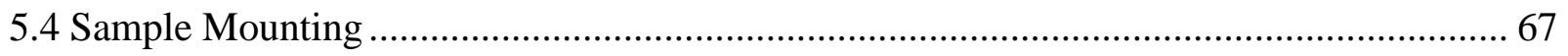

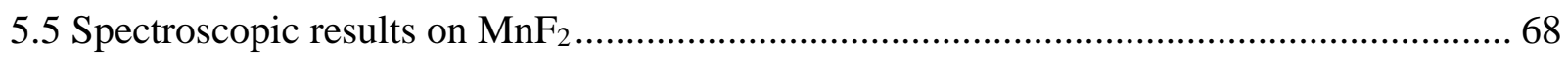

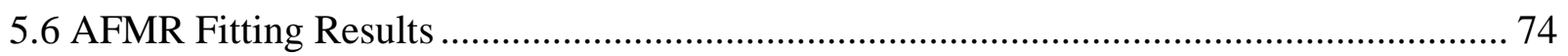

5.7 Theoretical calculation of the net magnetization ............................................................. 79

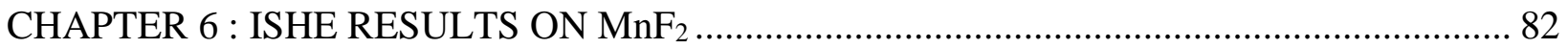

6.1 ISHE Voltage Measurement Setup ………………………………………………..... 82

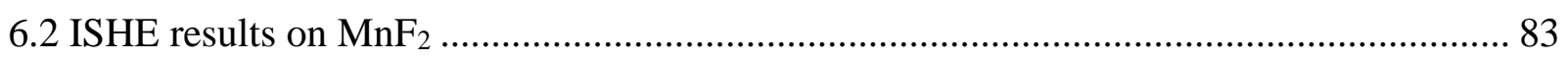

6.2.1 Coherent Spin Pumping vs Incoherent Spin-Seebeck Effect ..................................... 88

6.2.2 Spin-flop transition and the high magnetic field spin-flop mode ................................ 90

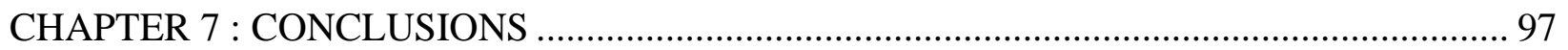

APPENDIX A: AFMR AND ISHE RESULTS FROM SAMPLES WITH $\mathrm{MgF}_{2}$ SUBSTRATE

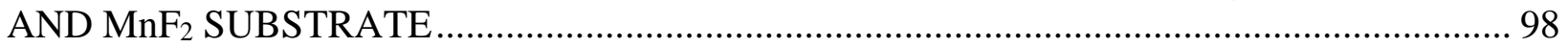

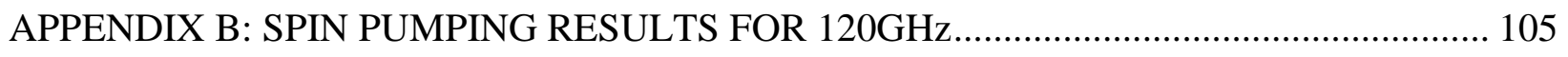

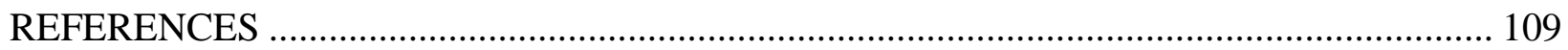




\section{LIST OF FIGURES}

Figure 1.1: Illustration of a spin valve in a magnetic recording head where the AF provides exchange bias and pins the magnetization of the FM reference layer while the FM sensor layer can have its magnetization switched electrically by charge current with spins polarized from the reference layer via spin-transfer torque. [4] ................................................................ 2

Figure 1.2: Crystal structure of $\mathrm{MnF}_{2}$ : $\mathrm{The} \mathrm{Mn}^{2+}$ ions form a body centered tetragonal unit cell. The arrows show the orientation of magnetic moments at each $\mathrm{Mn}^{2+}$ ion site. The body center $\mathrm{Mn}^{2+}$ ion is magnetically aligned antiparallel to the $\mathrm{Mn}^{2+}$ ions at the corner sites..................... 8 Figure 2.1: Temperature dependence of the magnetic susceptibility in AFMs. Below the Néel temperature, the spins in an AFM have antiparallel orientation. The susceptibility maximizes at the Néel temperature, as indicated by the prominent kink in the curve at $T=T N$ 10 Figure 2.2: Calculation of (a) perpendicular and (b) parallel susceptibilities of an antiferromagnet at zero temperature in the mean field approximation

Figure 2.3 : Magnetic susceptibility of $\mathrm{MnF}_{2}$, parallel and perpendicular to the tetragonal axis [51]

Figure 2.4 : Bethe-Slater curve for the magnitude of exchange integral as a function of interatomic distance. Most ferromagnets with positive exchange integral have a larger interatomic distance than the antiferromagnets with negative exchange integral. 18 Figure 3.1: Breit-Rabi diagram representing the splitting of energy levels in a spin $1 / 2$ system in an external field. Transitions between the two levels is possible by the absorption of a microwave radiation of a field-dependent frequency. 
Figure 3.2: Illustration of the precessional cone of cone angle $\Theta$ formed by torque exerted by an external field on a magnetic moment $\boldsymbol{\mu}$ 25

Figure 3.3: When the rf excitation field is turned off, the precessional motion is dampened by damping forces and the magnetization is brought back into equilibrium position before the excitation

Figure 3.4: Effective fields in the antiferromagnetic resonance of a uniaxial antiferromagnet. Each sublattice magnetization experiences the anisotropy field (acting along the z-axis in this case) and the exchange field due to the other sublattice. Together with the applied field, the effective field interacts with the sublattice magnetizations.

Figure 3.5: Resonance modes calculated by Kittel, Keffer and Nagamiya formalism of AFMR. The red and blue lines represent the Kittel-Keffer [56] described low field resonance mode, the red one being high frequency mode and the blue one being low frequency mode. The green line represent the spin flop mode calculated by Nagamiya [57] 35 Figure 3.6: Equilibrium orientations of sublattice magnetization at resonance. Two distinct modes exist: A low frequency mode (counter-clockwise) and a high frequency mode (clockwise) defined by the precessional direction. 37 Figure 3.7: Spin Flop Mode where the two magnetizations suddenly become orthogonal as well as canted towards the applied field giving rise to a net magnetization in the canted direction.... 38 Figure 3.8: Frequency vs external magnetic field relations of the AFMR signals in $\mathrm{MnF}_{2}$ as obtained by Hagiwara et. al. [21]. The full lines are theoretical fits for a field applied along the easy axis while the dash-dot lines are theoretical fits for a field applied perpendicular to easy axis. 
Figure 3.9: Nature of precessional cones of $\boldsymbol{M} 1$ and $\boldsymbol{M} 2$ in (1) the low field (a) high frequency (b) low frequency, (2a/b) spin flop transition and (3) spin flop mode. ..................................... 43

Figure 4.1: Illustration of (a) spin Hall effect and (b) inverse spin Hall effect ........................ 47

Figure 4.2: Illustration of the two types of current at the F/NM interface. The spin current pumped due to precessing magnetic moment in effective field diffuses along the spin diffusion length in the NM layer. The current due to backscattering is minimal for very conductive NM layer. 50

Figure 4.3: The eigenmodes associated with AFMR that show opposite chiralities. The illustration also shows how the staggered magnetization and the small net magnetization move with reference to the two magnetizations in each mode.

Figure 4.4: DC components of pumped spin current (black) and staggered spin current (orange) as functions of $\omega$ in units of $(\hbar e) G r(\gamma h a c) 2$ as calculated by Cheng et.al. for static field frequency $\omega H=0$, resonance frequency $\omega R=1 \mathrm{THz}$ and Gilbert damping $\alpha=0.01 .[45] \ldots \ldots .53$ Figure 4.5: For a fixed value of microwave power, the resonance value of Isdc scales with $\omega A \omega E$ which presents $\mathrm{FeF}_{2}$ with $\omega A \omega E \approx 0.6$ to be a better candidate for spin pumping than $\mathrm{MnF}_{2}$ with $\omega A \omega E<0.1 .[45]$

Figure 4.6: Illustration by Øyvind et. al. of precession of $\boldsymbol{m}$ and $\boldsymbol{n}$ in the AF that results in spins being pumped (Isp) into the NM. The resulting spin accumulation $\boldsymbol{\mu} s N$ in NM causes a backflow (Isb) of spins back into the AF. [46].... 55 Figure 4.7: Calculated (a/c) DC component (b/d) first harmonic AC component of the ISHE electric field as a function of external magnetic field along the easy axis of $\operatorname{MnF}_{2}(a, b)$ and 
$\mathrm{FeF}_{2}(\mathrm{c}, \mathrm{d})$. (b, d) shows different polarizations of the AC magnetic field. The AC field is $1 \mathrm{mT}$ and $\alpha=0.01[46]$ 57

Figure 4.8: Illustration of quantum mechanical spin-exchange processes at a FI/NM interface. The two graphics (a) and (b) show the magnon emission and absorption associated with the spinflip scattering of conduction electrons at the interface respectively, thus transferring spin angular momentum from FI to $\mathrm{N}$ and from $\mathrm{N}$ to FI through exchange interaction. [72] 59

Figure 5.1: Normalized axial electric field and azimuthal magnetic field as a function of radius for TM010 mode in a cylindrical cavity

Figure 5.2: Illustration of differences in resonance signal between over-coupled, critically coupled and under coupled resonant cavity

Figure 5.3: Experimental setup of the Quasi-Heterodyne Spectrometer at the National High Magnetic Field Laboratory, Tallahassee, FL[81] 65

Figure 5.4: Precut single crystal of $\mathrm{MnF}_{2}$ that was divided into four quadrants. Three were used for sample preparation and the fourth was kept for characterizations. The size of each quadrant is about $10 \mathrm{~mm}$ by $10 \mathrm{~mm}$. 66 Figure 5.5: $\mathrm{MnF}_{2}$ single crystal/ $\mathrm{MnF}_{2} 10 \mathrm{~nm}$ thin film/ $\mathrm{Pt} 4 \mathrm{~nm}$ heterostructure. On the left is the AFM image of the top layer of Pt which shows average RMS roughness of about $1.6 \mathrm{~nm}$. 67 Figure 5.6: (a) Sample mounted for EPR measurements. (b) Illustration of sample mounting with respect to the external field, microwave field and the easy axis of the sample. 68 Figure 5.7: Spin-pumping data and AFMR data positions for Sample 3 for $395 \mathrm{GHz}$ at low-field high frequency resonance. The red line is the AFMR spectra while the black line is the averaged spin-pumping data. The grey line is the raw spin-pumping signal. 70 
Figure 5.8: Spectroscopy results on Sample 1 for frequencies $120 \mathrm{GHz}, 240 \mathrm{GHz}$ and $336 \mathrm{GHz}$. The red circles denote non-reproducible experimental artefacts and do not contribute to the actual data.

Figure 5.9: Spectroscopic results on Sample 2 for frequencies $120 \mathrm{GHz}, 240 \mathrm{GHz}$ and $336 \mathrm{GHz}$. The red circle denote non-reproducible experimental artifacts and do not contribute to the actual data. 73

Figure 5.10: Spectroscopic results on Sample 3 for $120 \mathrm{GHz}$ and $395 \mathrm{GHz}$ 74

Figure 5.11: Calculated AFMR spectra for $\mathrm{MnF}_{2}$ with four different misalignment angles that are color coded as follows: Black $=0^{\circ}$, Red $=0.9^{\circ}$, Green $=3^{\circ}$, Blue $=7^{\circ}$ 76

Figure 5.12: AFMR data fitted with simulation for the three samples. The symbols represent AFMR data points while the solid lines are obtained from simulation. Each sample has a different misalignment angle and the zero misalignment data is also represented for comparison in each chart.

Figure 5.13: Net Equilibrium Magnetization in $\mathrm{MnF}_{2}$ : Projections of the net equilibrium magnetization $(M=M 1+M 2)$ in the $\mathrm{x}$ and $\mathrm{z}$ directions for the three misalignment angles $(\theta H)$ between the anisotropy axis $(H A \| z)$ and the applied field with the misalignment occurring in the x-direction. The grey dashed line represents $\theta H=90^{\circ}$.

Figure 6.1: Illustration of the device geometry for spin pumping and ISHE measurements. The misalignment angle $\theta H$ is to be minimized by rotating the sample.

Figure 6.2: ISHE signal for Sample 2 at $336 \mathrm{GHz}$. The voltage signal correspond to the resonance position observed in spectroscopic measurements. 85 
Figure 6.3: ISHE signal obtained in Sample 3 at 395GHz for (a) left-handed (b) right-handed circularly polarized microwaves and in Sample 2 at 240GHz for (c) left-handed (d) right-handed

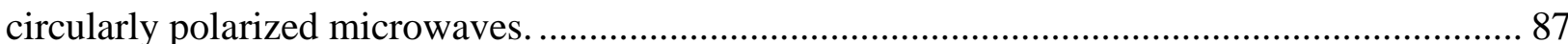

Figure 6.4: Power Dependence of the ISHE: (a) ISHE signal obtained at the Spin-Flop transition in Sample 3 at $395 \mathrm{GHz}$ for different powers of the microwave source and (b) corresponding power dependence with a linear fit. 88

Figure 6.5: Circular Polarization Modulation of Spin Pumping: Evolution of the ISHE signals with applied field as a function of the polarization of the microwaves for (a-f) $395 \mathrm{GHz}$ and (g-l) 240GHz. Polarization phases $0^{\circ}$ and $360^{\circ}$ correspond to right handed circular polarization and polarization phase $180^{\circ}$ corresponds to left handed circular polarization

Figure 6.6: Illustration of the types of handed-ness of the precession of the Néel vector based on the precession of the sublattice magnetizations. The external field is applied along the anisotropy axis.

Figure 6.7: Magnitudes of the ISHE signals for the LFM, SF and SFM resonances as a function of polarization value for both positive and negative field. 94 Figure 6.8: Illustration of the orientations of the sublattice magnetizations $\boldsymbol{M} 1$ and $\boldsymbol{M} 2$ and the applied field $\boldsymbol{H}$ with the anisotropy axis along the z-direction for four resonance points (1-2 for $395 \mathrm{GHz}$ and 3-4 for $240 \mathrm{GHz}$ ) representative of the changes in AF dynamics while transitioning from the high frequency AF resonance mode to the spin-flop mode through the spin-flop transition. The upper sketches represent the orientation and spin polarization of the pumped spin current and the induced ISHE electric field with respect to the measuring circuit in the sample. 
The lower insets illustrate the precessional cones of $\boldsymbol{M} 1$ and $\boldsymbol{M} 2$ for each resonance points

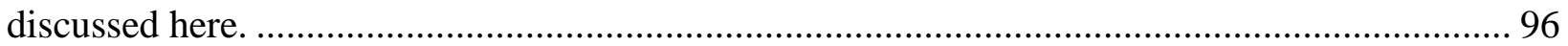




\section{LIST OF TABLES}

Table 1.1.Room temperature lattice parameters, Néel temperatures, next-nearest neighbor magnetic exchange interactions $\mathrm{J}_{\mathrm{nnn}}$ and uniaxial magnetic anisotropies $\mathrm{A}$ and $\mathrm{E}$ of $\mathrm{MnF}_{2} \ldots \ldots \ldots 8$ Table 5.1: List of samples used for the data presented in this thesis and the frequencies they were investigated at. 69

Table 5.2: Fitting parameters used in this thesis. 75 


\title{
LIST OF ABBREVIATION
}

\author{
AF Antiferromagnetic \\ EPR Electron Paramagnetic Resonance \\ F Ferromagnetic \\ HFM High Frequency Mode \\ ISHE Inverse Spin Hall Effect \\ LFM Low Frequency Mode \\ NM Non-magnetic \\ NMR Nuclear Magnetic Resonance \\ SF Spin-Flop \\ SFM Spin-Flop Mode \\ SHE Spin Hall Effect \\ STT Spin Transfer Torque
}




\section{CHAPTER 1 : INTRODUCTION}

This section gives the premise to this thesis and discusses the state-of-art in this field. The details of some of the topics discussed here will be presented in detail later on in this thesis.

\subsection{Antiferromagnetism and Spintronics}

Charge is a fundamental property intrinsic to electrons that has been harnessed for over a century for the manipulation and storage of information. Another intrinsic property associated with electrons is their intrinsic angular momentum commonly known as "spin". Movement of spin either in the form of spin-current or in the form of spin-polarized charge current has been the basis of the field of spintronics and ferromagnets with their magnetization have proved useful in this field. The magnetization direction of a ferromagnet can represent a bit of information with the orientation in the direction of field (up) as 1 and against the direction of field (down) as 0. Some of the central concepts of spintronics include generation and detection of spin current via dynamical Spin Pumping and Inverse Spin Hall Effect (ISHE) respectively and spin transport via Spin-Transfer Torque (STT) and Spin Hall Effect (SHE). Recently though, the field of spintronics has been focused on reducing power consumption and scale [1,2] and antiferromagnets have great possibility in this regard.

In this context, some unique characteristics of antiferromagnets become interesting. In particular, antiferromagnets present a long-range ordering causing the magnetic moments to align in a regular 
pattern with their neighboring magnetic moments pointing in opposite directions and resulting in zero net magnetization. The implication of this is that antiferromagnets do not have stray fields and are practically immune to external magnetic field perturbations. The dynamics of antiferromagnets are also much faster than that of ferromagnets: A strong exchange field coupled with anisotropy give typical antiferromagnets resonance frequencies in the Terahertz regime. These qualities have pushed antiferromagnets into the limelight as suitable candidates for faster, compact, and more efficient materials for spintronic applications [3].

\section{Magnetic recording head}

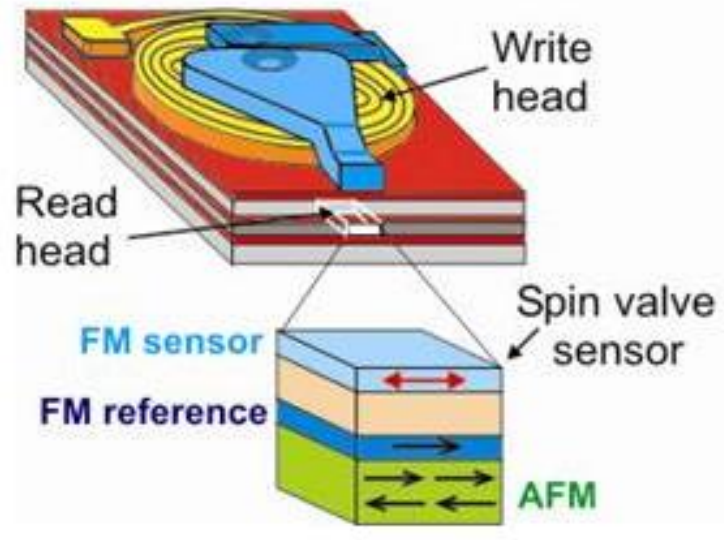

Figure 1.1: Illustration of a spin valve in a magnetic recording head where the AF provides exchange bias and pins the magnetization of the FM reference layer while the FM sensor layer can have its magnetization switched electrically by charge current with spins polarized from the reference layer via spin-transfer torque. [4] 
Antiferromagnets have been considered suitable only for theoretical considerations as put forth by Louis Néel in this Nobel lecture from 1970 [5], until the early 1990s when exchange bias found its place in large scale industrial application in recording heads of memory devices (hard disks, Figure (1.1))[6-8]. Indeed, antiferromagnets have been used commercially to provide exchange bias to pin down the magnetization of reference ferromagnetic layer in spin valves. Presently, because of the promising properties of antiferromagnets, efforts are being made at looking into the possibility of replacing ferromagnets with antiferromagnets as active elements and creating efficient and stable devices with antiferromagnets are the major components.

\subsection{Antiferromagnetic Materials}

Antiferromagnetic materials are more abundant than ferromagnets and are mostly found in the form of transition metal oxides, halides and metal alloys. They exist in both metallic and insulator forms. Metallic AFs are mostly Mn-based alloys such as IrMn, PtMn and FeMn, relatively easy to fabricate using sputtering techniques, hence their widespread use in industrial applications. They have also been used for the study of spintronic concepts such as the spin Hall effect [9-11], spin pumping [12, 13], spin-transfer effects [14] and the spin transfer torque [15]. Oxides and halides of transition metals constitute the respective families of insulating AFs and they have recently attracted significant attention from theorists and experimentalists alike since they offer substantially lower damping (losses) than their metallic counterparts. The oxides are ideal for studying magnonic and caloritronic effects such as spin wave propagation and spin Seebeck effect [16-18]. The halides such as $\mathrm{MnF}_{2}$ [19] and $\mathrm{FeCl}_{2}$ [20] exhibit spin-flop and spin-flip transitions 
respectively. In particular $\mathrm{MnF}_{2}$ exhibits this transition below 10T making it desirable for experiments. These transitions allow control of the antiferromagnetic resonant mode degeneracy [21] and have been used to demonstrate the spin Seebeck effect [16] and dynamical spin pumping (this thesis).

In addition to these, antiferromagnetic semi-conductors (MnTe, [22]) and semi-metals (CuMnAs, [23]) have shown great promise. CuMnAs has been used to create the first ferro-magnet-free memory prototypes recently that utilizes inverse spin galvanic effect and planar Hall effect for writing and reading [23]. Moreover, antiferromagnetic order was found in topologically nontrivial phases of matter such as Weyl semimetals GdPtBi [24] and CuMnAs [25] spurring a whole new field of topological antiferromagnetic spintronics [26].

\subsection{Control and Manipulation of Antiferromagnetic Order}

The question of how to control and manipulate the antiferromagnetic order began as soon as the antiferromagnetic order was discovered and studied. Since then significant advancements have been made in developing techniques to achieve this goal.

\subsubsection{Manipulation by Magnetic Field}

Currently, the antiferromagnetic order can be manipulated by several different methods and one of them is the application of a magnetic field (Stoner-Wohlfarth model) which can reorient an 
antiferromagnetic sublattice and can help determining the magnetic anisotropy of the system. Such magnetic field manipulation is quite evident in $\mathrm{MnF}_{2}$ [19] and $\mathrm{FeCl}_{2}$ [20], where spin-flop and spin-flip transitions can be induced by the application of the field. The effect of magnetic field on the dynamics of a uniaxial antiferromagnet will be discussed at length in Chapter 3.

\subsubsection{Manipulation by Light}

Antiferromagnets are able to display linear dichroism due to the breaking of time-reversal symmetry resulting in the absorption of linearly polarized light based on the orientation of the Néel order parameter. This property has been utilized to control the AF order parameter in $\mathrm{MnF}_{2}$ [27], $\mathrm{NiO}$ [28] and very recently in $\mathrm{HoFeO} 3$ [29] where inertial switching is observed. Very high frequency $\mathrm{AF}$ modes of $\mathrm{NiO}$ [3], $\mathrm{MnO}$ [30] and others have been achieved through optical excitation leading to the study of AF spin-wave modes using femto-magnetism and $\mathrm{THz}$ stimuli [31]. Efforts are also being made to generate $\mathrm{THz}$ radiation using spin polarized charge current or spin current in AF systems [32].

\subsubsection{Manipulation by exchange bias}

Just as antiferromagnets can be used to pin the magnetization of ferromagnets using exchange bias, conversely exchange bias can be utilized to manipulate the antiferromagnetic order. In fact, exchange bias can be used to study current-induced antiferromagnetic order manipulation (spintransfer torque) as well as its Onsager reciprocal - spin pumping, which is the focus of this thesis. These will be discussed in detail in Chapter 4. Spin-transfer torque in antiferromagnets was predicted first by Nunez [33]. Since then attempts both in calculations [34-37] and experiments 
$[38,39]$ have been made to realize AF spin valves and AF tunnel junctions. One of the challenges in realizing spin-transfer torque in AF-based devices experimentally is that the current-induced changes of the AF order are present only when the current is present. The AF system goes back to its original state as soon as the inducing current is removed just as in an exchange spring [38]. Current-driven AF dynamics has also gathered attention in recent times under the context of exploiting damping-like torque to induce spin dynamics in AF systems with uniaxial and biaxial anisotropies [40-42]. In addition, inertial switching of an antiferromagnet with uniaxial anisotropy under a current pulse has been theoretically investigated by Cheng et.al. [43].

Reciprocal to spin-transfer torque, spin pumping has been elusive in AF systems to this date. Ross et.al. [44] attempted at observing spin-pumping in insulating $\mathrm{MnF}_{2}$ at low frequencies but failed. The possible reasons have been given by Cheng et. al [45] and Øyvind et. al [46]. Cheng et. al. [45] attribute the very low microwave absorption efficiency at resonance point in $\mathrm{MnF}_{2}$ as the reason for the suppression of the signals while Øyvind et. al [46] point out the polarization dependence of electric signal from spin pumping in uniaxial antiferromagnets like $\mathrm{MnF}_{2}$ and $\mathrm{FeF}_{2}$. The much larger microwave absorption efficiency of $\mathrm{FeF}_{2}[45]$ has made it a promising candidate albeit the dynamics of this system is in the $\mathrm{THz}$ regime. Other approaches to manipulate the AF order parameter have also been explored and include the spin-orbit torques [47], the inverse spin galvanic torque [23, 48], the spin Hall torque [49] and the spin Seebeck effect [16]. 


\subsection{Uniaxial Antiferromagnet $\mathrm{MnF}_{2}$}

$\mathrm{MnF}_{2}$ is a uniaxial antiferromagnet with a well-defined easy axis in the c-direction. The crystal structure is of rutile nature as shown in Figure 1.2, belonging to the tetragonal space group $D_{4 h}^{14}$ with two molecules per unit cell. The lattice parameters of $\mathrm{MnF}_{2}$ are tabulated in Table 1.1. Neutron scattering study below the Néel temperature [50] have revealed the magnetic structure of $\mathrm{MnF}_{2}$. Below the Néel temperature, the spins at the body center sites point antiparallel to those the corner sites with the spin easy axis parallel to the c-axis. The orbital angular momentum of the $\mathrm{Mn}^{2+}$ ion in $\mathrm{MnF}_{2}$ is essentially zero and thus the weak anisotropy is dominated by dipole-dipole interactions. This coupled with a large isotropic exchange force produces a large anisotropic field resulting in a stable magnetic configuration of spins aligning along the tetragonal axis [50] and causing the zero-field uniform antiferromagnetic resonance (AFMR) uniform mode to occur at a relative low frequency of $261 \mathrm{GHz}$. The dynamic response of this system will be described in detail in Chapter 3. 


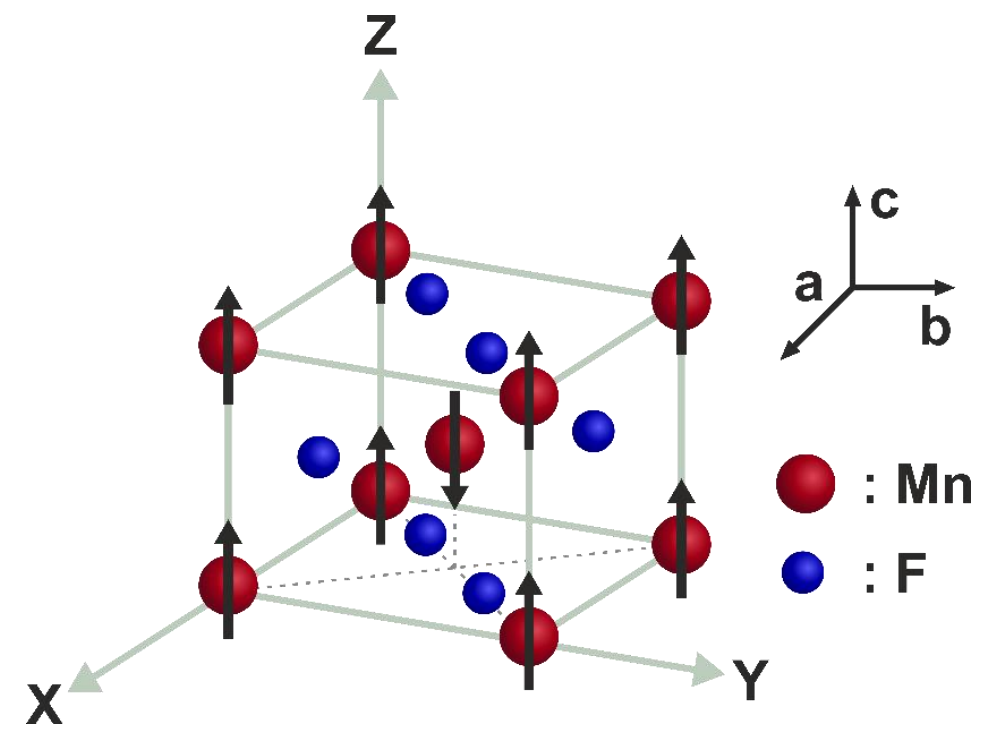

Figure 1.2: Crystal structure of $\mathrm{MnF}_{2}$ : The $\mathrm{Mn}^{2+}$ ions form a body centered tetragonal unit cell. The arrows show the orientation of magnetic moments at each $\mathrm{Mn}^{2+}$ ion site. The body center $\mathrm{Mn}^{2+}$ ion is magnetically aligned antiparallel to the $\mathrm{Mn}^{2+}$ ions at the corner sites.

Table 1.1.Room temperature lattice parameters, Néel temperatures, next-nearest neighbor magnetic exchange interactions $J_{n n n}$ and uniaxial magnetic anisotropies $A$ and $E$ of $M n F_{2}$.

\begin{tabular}{|l|l|l|l|l|l|l|l|}
\hline \multirow{2}{*}{$\mathbf{M n F}_{2}$} & $\mathbf{a}(\mathbf{n m})$ & $\mathbf{c}(\mathbf{n m})$ & Spin & $\mathbf{T}_{\mathbf{N}}(\mathbf{K})$ & $\mathbf{J}_{\text {nnn }}(\mathbf{m e V})$ & $\mathbf{A}(\mathbf{m e V})$ & $\mathbf{E}(\mathbf{m e V})$ \\
\cline { 2 - 8 } & 0.4873 & 0.3310 & $5 / 2$ & 67.0 & 0.304 & -0.092 & - \\
\hline
\end{tabular}




\section{CHAPTER 2 : ANTIFERROMAGNETIC THEORY}

This section provides the physical background on antiferromagnetism. It also presents the different fields contributing to the free energy of an antiferromagnetic system.

\subsection{Introduction}

Antiferromagnets are a class of magnetic materials with a distinct feature about them: their magnetic moments are Weiss field coupled with a negative exchange integral, which sets them apart from ferromagnets. This feature also renders them with a zero net magnetization at equilibrium as the neighboring spins are oppositely ordered by a long range ordering. Initiated and extensively studied by Néel, the theory of antiferromagnetism has been expanded by van Vleck, Nagamiya and others. In most cases, the AF crystal lattice is modeled as a combination of two sublattices: one with positive spins and the other with negative spins at absolute zero and just as in ferromagnets, the ordering transition occurs at a thermal point called as Néel temperature. Below this temperature, the long range magnetic ordering is preserved by a magnetic energy resulting from anisotropy and exchange interactions.

\subsection{Magnetic Properties}

Kittel[51] describes antiferromagnetism as a special case of a ferrimagnet where both the sublattices have equal saturation magnetization and the Néel temperature in the mean field 
approximation is given by $T_{N}=\mu C$, where $C$ is the Curie constant for a single lattice. He gives the susceptibility of an AFM in the paramagnetic region of $T>T_{N}$ as $\chi=\frac{2 C}{T+T_{N}}$ whereas the experimental results at $T>T_{N}$ are of the form $\chi=\frac{2 C}{T+\theta}$, where the value of $\frac{\theta}{T_{N}}$ differs from unity and can be obtained after considering next-nearest-neighbor interactions[51].

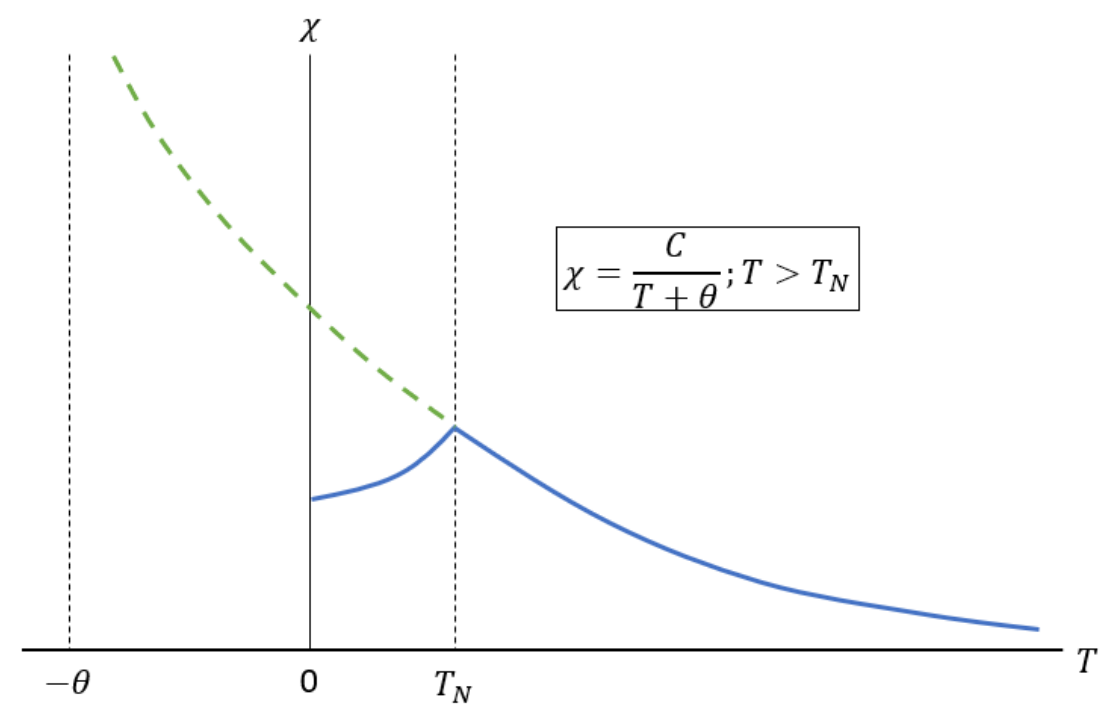

Figure 2.1: Temperature dependence of the magnetic susceptibility in AFMs. Below the Néel temperature, the spins in an AFM have antiparallel orientation. The susceptibility maximizes at the Néel temperature, as indicated by the prominent kink in the curve at $T=T_{N}$.

Kittel describes two unique situations: $i$ ) when the external field is perpendicular to the axis of the spins, and $i i)$ when the field is parallel to the axis, subsequently describing two susceptibilities $\left(\chi_{\|}\right.$ and $\chi_{\perp}$ ) below the Néel temperature. Above the Néel temperature the susceptibility is nearly 
independent of the direction of the field relative to the spin axis. He gives an elementary method within mean field approximation, of obtaining these susceptibilities where he defines the energy density in the presence of external field $\boldsymbol{H}$ as

$$
U=\left(\boldsymbol{M}_{+} \cdot \boldsymbol{M}_{-}\right)-\boldsymbol{H} \cdot\left(\boldsymbol{M}_{+}+\boldsymbol{M}_{-}\right) \cong-M^{2}\left[1-\frac{1}{2}(2 \varphi)^{2}\right]-2 H M_{\varphi}
$$

where $2 \varphi$ is the angle between the two spins and $M=\left|M_{+}\right|=\left|M_{-}\right|$.

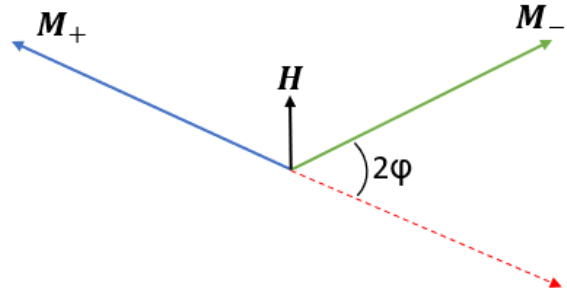

(a)

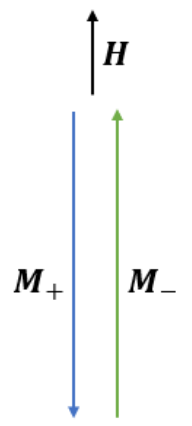

(b)

Figure 2.2: Calculation of (a) perpendicular and (b) parallel susceptibilities of an antiferromagnet at zero temperature in the mean field approximation

Minimizing the energy yields $\varphi=\frac{H}{2 M}$ which then gives the perpendicular susceptibility as

$$
\chi_{\perp}=\frac{2 M \varphi}{H}
$$


The parallel susceptibility at zero temperature is zero as the magnetic energy in this orientation is not changed if the spins make equal angles with the field. Thus

$$
\chi_{\|}(0 K)=0
$$

The parallel susceptibility increases smoothly with temperature up to the Néel temperature while the perpendicular susceptibility stays constant. Beyond this point the susceptibility follows a paramagnetic pattern, decreasing steadily with temperature. An example of this is observed in $\mathrm{MnF}_{2}$ (Figure 2.3).

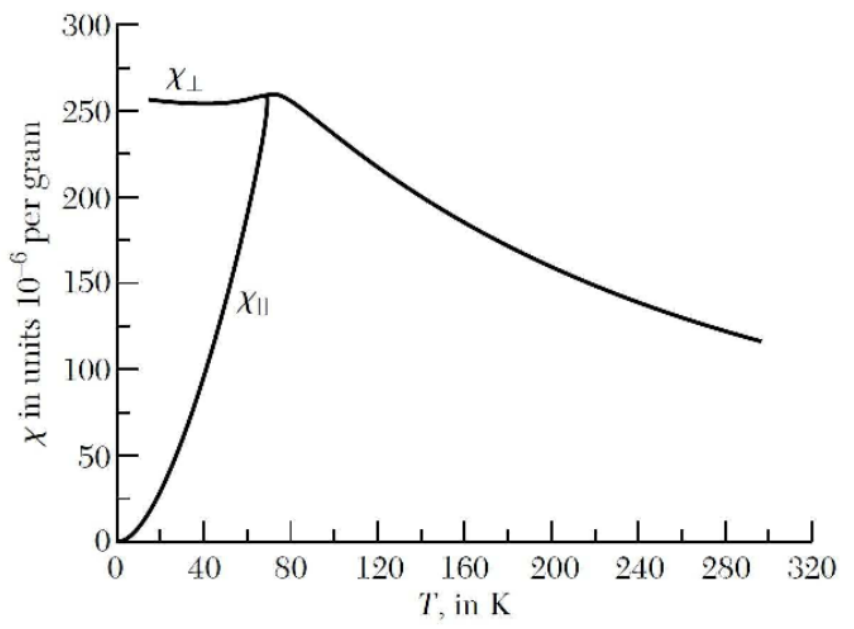

Figure 2.3 : Magnetic susceptibility of $\mathrm{MnF}_{2}$, parallel and perpendicular to the tetragonal axis [51] 
In the van Vleck theory, each type of spin $\left(\boldsymbol{S}^{ \pm}\right)$in an AFM is subjected to an effective field arising from a molecular magnetic field due to the surrounding $\boldsymbol{S}^{\mp}$ spins and the external magnetic field and is given as

$$
\boldsymbol{H}_{e f f}^{ \pm}=\boldsymbol{H}+2 J z g^{-1} \beta^{-1} \overline{\boldsymbol{S}}^{\mp}
$$

where,

$J$ is the negative exchange integral

$z$ is the number of nearest neighbors

$g=2$ is the Landé factor

$\beta=\frac{h e}{4 \pi m c}$ is the Bohr magneton

$\boldsymbol{H}$ is the external field, and

$\overline{\boldsymbol{S}}^{\mp}$ is the statistical average of $\boldsymbol{S}^{ \pm}$at a given T

van Vleck assumes $\overline{\boldsymbol{S}}^{\mp}$ and $\boldsymbol{H}_{e f f}^{ \pm}$to be in the same direction. Thus

$$
g \beta \overline{\boldsymbol{S}}^{ \pm}=\chi^{ \pm} \boldsymbol{H}_{e f f}^{ \pm}
$$

There is an exception where $\overline{\boldsymbol{S}}^{ \pm}$are perpendicular to the external field for vanishing field strength given by

$$
\chi^{+}=\chi^{-}=\frac{1}{-2 J z g^{-2} \beta^{-2}}
$$

This gives a magnetization per unit volume $\boldsymbol{M}$ as 


$$
\boldsymbol{M}=\frac{1}{2} N g \beta\left(\overline{\boldsymbol{S}}^{+}+\overline{\boldsymbol{S}}^{-}\right)=\frac{N g^{2} \beta^{2}}{-4 J z} \boldsymbol{H}
$$

where $N$ is the number of atoms per unit volume.

van Vleck assumes that the parallel and perpendicular domains in an AFM exist in the ratio of 1:2 and gives the susceptibility formula $\chi=\frac{\chi_{\|}+2 \chi_{\perp}}{3}$. Nagamiya argues that this assumption cannot be justified as the difference in free energy per unit volume between the two domains, namely $\frac{1}{2}\left(\chi_{\|}-\chi_{\perp}\right) H^{2}$, is positive implying only perpendicular domains exist under thermal equilibrium and thus a constant susceptibility below the Curie point, which contradicted experimental observations. Further Nagamiya explains that while the anisotropy energy is of the same order in both the ferromagnets and antiferromagnets, the external field energy $(-\boldsymbol{M} \boldsymbol{H})$ in ferromagnets can be greater than the anisotropy energy allowing the magnetization $\boldsymbol{M}$ to be easily turned in the direction of the external field while in antiferromagnets this energy, given as $-\frac{1}{2} \chi H^{2}$, is so small that the spontaneous magnetization of the two sublattices can hardly deviate from the easy axis for ordinary field strength. This calls for a larger role of anisotropy in the antiferromagnetic theory and thus, Nagamiya defines one or many mutually equivalent axes of easy magnetization about which the anisotropy energy has rotational symmetry for small angles of deviation. If $z$-axis were to be considered one of such axes, then the anisotropy energy would be expressed in terms of the perpendicular direction cosines $\alpha^{ \pm}$and $\beta^{ \pm}$as

$$
F=\frac{1}{2} K\left[\left(\alpha^{+2}+\beta^{+2}\right)+\left(\alpha^{-2}+\beta^{-2}\right)\right]
$$


where $K$ is the anisotropy constant and can be of different types such as uniaxial, cubic, etc. The effective magnetic field acting upon $\boldsymbol{M}^{ \pm}$due to this energy is given as

$$
-\frac{K}{M_{o}}\left(\alpha^{ \pm}, \beta^{ \pm}, 0\right)
$$

Defining $A=\frac{-4 \mathrm{Jg}^{-2} \beta^{-2}}{N}$, Nagamiya gives a total magnetization as

$$
M=M^{+}+M^{-}=\left(0, \frac{H \beta_{H}}{A}, 0\right)
$$

and the parallel and perpendicular susceptibilities as

$$
\chi_{\|}=0, \chi_{\perp}=\frac{1}{A}
$$

The theories developed by van Vleck and Nagamiya both point towards the same susceptibility formula except for the essential difference in the definition of parallel and perpendicular directions. While van Vleck defines them as the directions of spontaneous magnetization, Nagamiya improves upon it by addressing them as the axes of easy magnetization.

\subsection{Spin Flop Transition}

A characteristic feature of easy axis antiferromagnets is that they undergo a field dependent first order phase transition where, under the effect of an external field applied along the easy axis, the oppositely directed magnetic moments break symmetry and reorient themselves perpendicular to 
the applied field, aptly named spin flop. First theorized by Néel for classical two-sublattice based antiferromagnets with sufficiently weak anisotropy in 1936, it was observed fifteen years later in 1951 in $\mathrm{CuCl}_{2} \cdot 2 \mathrm{H}_{2} \mathrm{O}$. The spin flop transition has been observed in many types of antiferromagnets since then. Even though the term spin flop was originally strictly used for two-sublattice collinear easy-axis AFMs, the term has now expanded its definition to include such transition in other AFMs caused by similar field driven mechanisms such as AFMs with Dzyaloshinskii-Moriya interaction, in multi-sublattice AFMs and in noncentrosymmetric AFMs. More detailed treatment of this phenomenon will be done in Chapter 3.

\subsection{Energy contributions in a uniaxial Antiferromagnet}

In a uniaxial AFM, the most dominant energy contributions arise from the following interactions:

\subsubsection{Exchange interaction}

This is a quantum mechanical coupling between the magnetic moments of identical particles like electrons by virtue of which the individual moments either align parallel or antiparallel to each other giving rise to magnetic states such as ferromagnetism, antiferromagnetism and ferrimagnetism. The charge distribution of a system of two spins depends on the relative orientation between them as per Pauli exclusion principle and the electrostatic energy difference between the two defines the exchange energy. The three main types of exchange interactions are i) direct exchange, ii) double exchange and iii) superexchange. 
Direct exchange interaction is a very strong interaction between nearest neighbor cations but exponentially decays with interatomic distance. In the Heisenberg model, this interaction can be represented for a system of two electron spins as:

$$
\widehat{H}_{e x}=-2 J_{e x} \hat{S}_{1} \hat{S}_{2}
$$

where $\hat{S}_{1}, \hat{S}_{2}$ are the spin operators and $J_{e x}$ is the exchange integral (or overlap) integral. Direct exchange exists between magnetic moments placed close enough for their wavefunctions to sufficiently overlap. For larger interatomic distances, $\quad J_{e x}>0$ and parallel alignment for ferromagnetic systems comes into existence. For smaller interatomic distances, $J_{e x}$ is negative and antiferromagnetic and ferrimagnetic systems are formed. This is represented by the Bethe-Slater curve for the magnitude of direct exchange as a function of interatomic distance (Figure 2.4)[52, 53].

Double exchange is a mechanism of indirect exchange coupling between moments in different oxidation states over relatively large distances and was first proposed by Zener(1951) who theorized that in mixed crystals of materials such as $\mathrm{LaMnO}_{3}$ and $\mathrm{BaMnO}_{3}$, ferromagnetic alignment occurs due the lowered energy resulting from the fact that electron transfer from one species to another does not have to involve a change in spin direction in order to conform with Hund's rule. This idea is superficially similar to superexchange where a similar indirect exchange interaction occurs. Also known as Kramers-Anderson superexchange, this coupling is predominantly observed in antiferromagnets between cations that are next-nearest neighbor through a non-magnetic anion (for example the oxygen in $\mathrm{MnO}$ ). Here the two involved cations 
are in the same oxidation state as opposed to double exchange. If the involved cations are connected to the intermediary anion at 90 degrees, then the alignment can be ferromagnetic. A limiting case of double exchange interaction is the Ruderman-Kittel-Kasuya-Yoshida (RKKY) interaction where coupling occurs between nuclear magnetic moments or localized inner d- or fshell electron spins in a metal through conduction electrons.

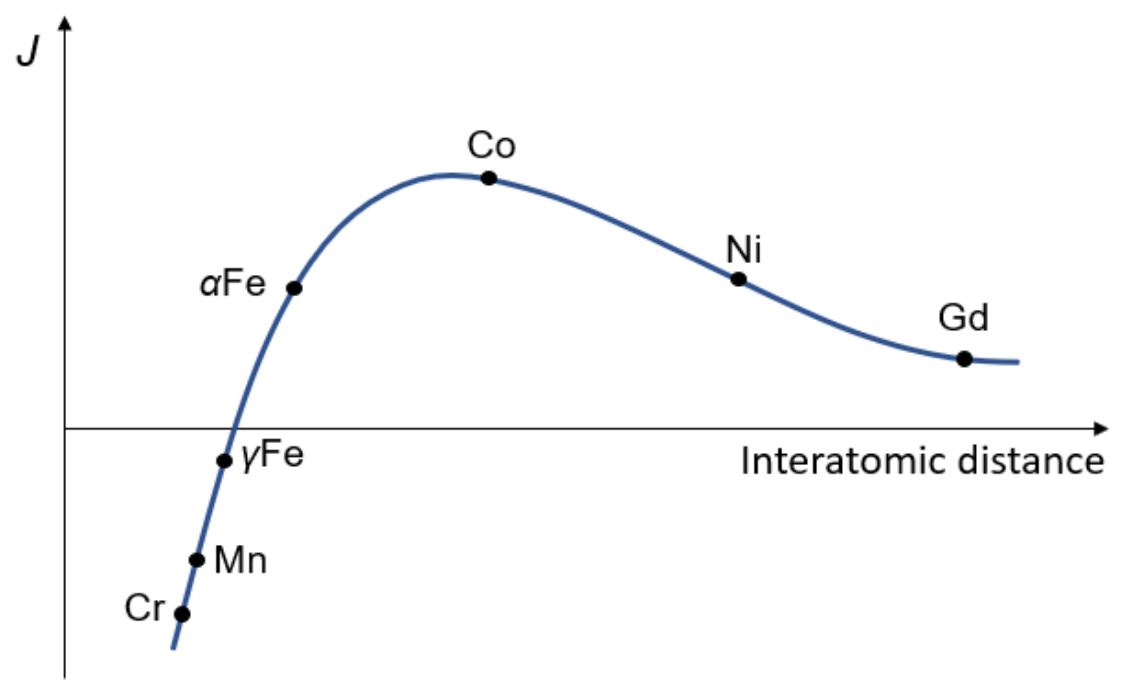

Figure 2.4: Bethe-Slater curve for the magnitude of exchange integral as a function of interatomic distance. Most ferromagnets with positive exchange integral have a larger interatomic distance than the antiferromagnets with negative exchange integral.

In $\mathrm{MnF}_{2}$, the dominant exchange interaction is the direct exchange where the free energy term associated with the exchange field $\boldsymbol{H}_{E}$ is $\Lambda \mu_{o} M_{1} . M_{2}$, with $\mu_{o} H_{E}=\Lambda M, M$ being the magnitude of the magnetic moments. 


\subsubsection{Anisotropy}

Crystals of ferromagnets and antiferromagnets display intrinsic magnetic anisotropy. This anisotropy can be considered to originate from three sources: 1) dipolar interaction between magnetic moments, 2) anisotropic exchange interaction or pseudo-dipolar interaction (van Vleck, 1937b,1951), which is a combined effect of LS coupling and exchange interaction and 3) crystalline field anisotropy. When a free magnetic ion feels the crystalline electric field arising from surrounding positive and negative ions, Stark effect causes the directional degeneracy of the orbital angular momentum $\mathbf{L}$ to be partially or completely lifted and the ground state magnetic moment can be considered as spin (S) magnetic moment. However, the anisotropic off-diagonal elements of spin angular momentum vector $\mathbf{S}$ still contribute to the ground state magnetic moment as a perturbation which makes the magnetic moment possessed by a magnetic ion and consequently the g-value to be anisotropic.

The three origins of anisotropy discussed above give rise to anisotropy energy not only in the AF state but also anisotropy energy to susceptibility above the Néel temperature. In the AF state, the spin lattice is divided into two sublattices with one being occupied by plus spins and the other by

minus spins. The direction of the common axis of these plus and minus spins with respect to the crystal axes is fixed by the anisotropy energy. 
The anisotropy of $\mathrm{MnF}_{2}$ has been studied by Yoshida (1951) and Keffer (1952). In particular Keffer concluded that the main contributor to the anisotropy energy in $\mathrm{MnF}_{2}$ is the magnetic dipolar energy. The free state of $\mathrm{Mn}^{2+}$ is ${ }^{6} S(3 d)^{5}$ with no orbital degeneracy. However, van Vleck and Penney (1934) have shown that the crystalline field and spin-orbit coupling also contribute to the anisotropy energy in $\mathrm{MnF}_{2}$. Thus, the anisotropy energy in the crystal of $\mathrm{MnF}_{2}$ can be represented in the following form:

$$
\begin{array}{cc}
D_{\alpha} S_{\alpha}^{2}+D_{\beta} S_{\beta}^{2}-\left(D_{\alpha}+D_{\beta}\right) S_{\gamma}^{2} & \text { for the corner ion } \\
D_{\beta} S_{\alpha}^{2}+D_{\alpha} S_{\beta}^{2}-\left(D_{\alpha}+D_{\beta}\right) S_{\gamma}^{2} & \text { for the body-center ion }
\end{array}
$$

where $\mathrm{MnF}_{2}$ as a rutile crystal is modeled to have a body-centered tetragonal lattice and $\alpha, \beta, \gamma$ represent the principal axes of the crystalline field at each site. The anisotropy energy calculations performed by Keffer based on the superstructure of spins determined by neutron diffraction study by Erickson and Shull (1951) and Shull (1953) show the corner sited occupied by plus spins and the body-centered sites by minus spins.

In a phenomenological approach, the effect of anisotropy can be interpreted in the form of an anisotropy energy. Anisotropy in magnetization and magnetic properties lead to the existence of an easy axis of magnetization which is the axis along which spins align in the absence of an external field. If $\theta_{1}$ and $\theta_{2}$ are the angles made by the magnetization vectors of the two sublattices, the free energy associated with anisotropy in the case of a uniaxial crystal like that of $\mathrm{MnF}_{2}$ can be written as: 


$$
F_{a}=\left(\frac{K}{2}\right)\left(\sin ^{2} \theta_{1}+\sin ^{2} \theta_{2}\right)
$$

\subsubsection{Zeeman Interaction}

Like any magnetic system, antiferromagnets experience Zeeman interaction when placed in an external magnetic field. The application of a static magnetic field on a system of electrons causes the energy level of the system to split into two or more components between which transitions are possible upon application of suitable electromagnetic radiation. This effect is known as the Zeeman effect and is the basis upon which applications involving resonance spectroscopy are developed. Detailed mathematical treatment of Zeeman interaction in case of uniaxial antiferromagnet will be done in Chapter 3. The free energy associated with Zeeman interaction in the case of a two-sublattice uniaxial antiferromagnet can be written as:

$$
F_{\text {Zeeman }}=-\mu_{o} \boldsymbol{H}_{o} .\left(\boldsymbol{M}_{1}+\boldsymbol{M}_{2}\right)
$$




\section{CHAPTER 3 : MAGNETIC RESONANCE THEORY}

This section deals with the theory behind magnetic resonance and its quantum mechanical treatment starting with an electron spin system, extending to ferromagnetic systems and analogically antiferromagnetic systems

\subsection{Electron Paramagnetic Resonance}

Electron Paramagnetic Resonance (EPR) is a very useful technique of spectroscopy to investigate a wide range of problems in fundamental and applied sciences such as understanding fundamental physics, characterization and study of molecules and compounds in material and earth sciences as well as biology and medicine. It is in principle similar to Nuclear Magnetic Resonance (NMR) spectroscopy except the obvious difference of the use of electron spin in EPR instead of nuclear

spin in NMR. The basic idea behind EPR is to utilize the resonant state of electron spin to absorb electromagnetic radiation of a suitable frequency.

An electron is associated with an intrinsic angular momentum conveniently called spin which has a value of $1 / 2$. Thus, a free electron is best described as a spin-half (1/2) system. A spin-half system with magnetic moment $\boldsymbol{\mu}$ has two energy levels described by their spin states: Up $(+1 / 2)$ and Down $(-1 / 2)$ and in the absence of any external field, they are degenerate. When placed in an external magnetic field $H_{o}$, this degeneracy is lifted as the two states split in energy levels as illustrated in Figure 3.1. 


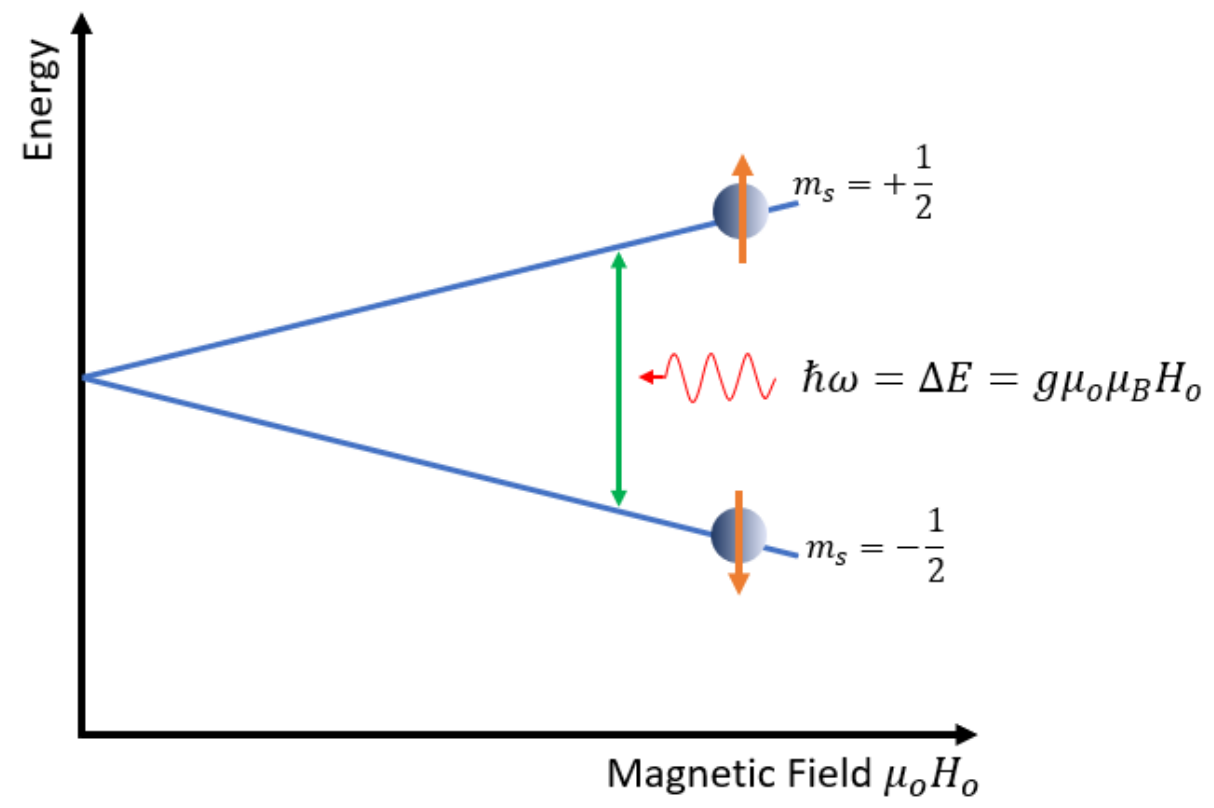

Figure 3.1: Breit-Rabi diagram representing the splitting of energy levels in a spin $1 / 2$ system in an external field. Transitions between the two levels is possible by the absorption of a microwave radiation of a field-dependent frequency.

This phenomenon is known as Zeeman splitting and the Zeeman energy term is given as:

$$
H_{\text {Zeeman }}=-\boldsymbol{\mu} \cdot \mu_{o} \boldsymbol{H}_{o}
$$

The corresponding eigenstates quantized by the magnetic quantum number $m_{j}$ are given as:

$$
E_{k}=g \mu_{o} \mu_{B} m_{k} H_{o}
$$

The size of the gap between $i$ and $j$ states is thus $\Delta E=E_{i}-E_{j}=g \mu_{o} \mu_{B} H_{o}$ which increases with increasing field. Transition between these two states can be achieved by irradiating the system with microwaves of frequency $\omega$ that satisfy the condition: 


$$
\hbar \omega=\Delta E=g \mu_{o} \mu_{B} H_{o} ; \Delta m_{k}= \pm 1
$$

The polarization of microwave field $\boldsymbol{H}_{r f}$ that allow such transition has to be perpendicular to $\boldsymbol{H}_{\boldsymbol{o}}$ which means that the spin resonance is excited only if the polarization of the electromagnetic wave possesses r.f. magnetic field components orthogonal to the applied magnetic field.

Interestingly Vonsovskii showed in his book that such a resonance condition can be derived classically. When a magnetic moment $\boldsymbol{\mu}$ is placed in an external magnetic field $\boldsymbol{H}_{\boldsymbol{o}}$, it experiences a torque $\mathbf{T}$ that works to align the magnetic moment in the direction of the magnetic field:

$$
\mathbf{T}=\boldsymbol{\mu} \times \mu_{o} \boldsymbol{H}_{o}
$$

Utilizing Newton's second law of motion for angular momentum $\frac{d \boldsymbol{L}}{d t}=\mathbf{T}$ and also the fact that $\boldsymbol{\mu}=$ $-\gamma \boldsymbol{L}$, where $\gamma=\frac{e g}{2 m_{e}}$ is the gyromagnetic ratio and the negative sign is for electronic charge, the equation of motion of a magnetic moment in an external magnetic field comes out to be:

$$
\frac{d \boldsymbol{\mu}}{d t}=-\gamma \boldsymbol{\mu} \times \mu_{o} \boldsymbol{H}_{o}
$$

The solution to Eqn. (3.5) yields a precessional motion with a frequency known as Larmor frequency given by $\omega_{L}=\gamma \mu_{o} H_{o}$. Microwave radiation of this frequency is absorbed at resonance and the precessional cone angle $\Theta$ (Figure 3.2) will be enhanced, ultimately leading to a complete spin reversal. The resonance condition here is the same as the one obtained quantum mechanically by utilizing the transformation: $\gamma=\frac{g \mu_{B}}{\hbar}$. 


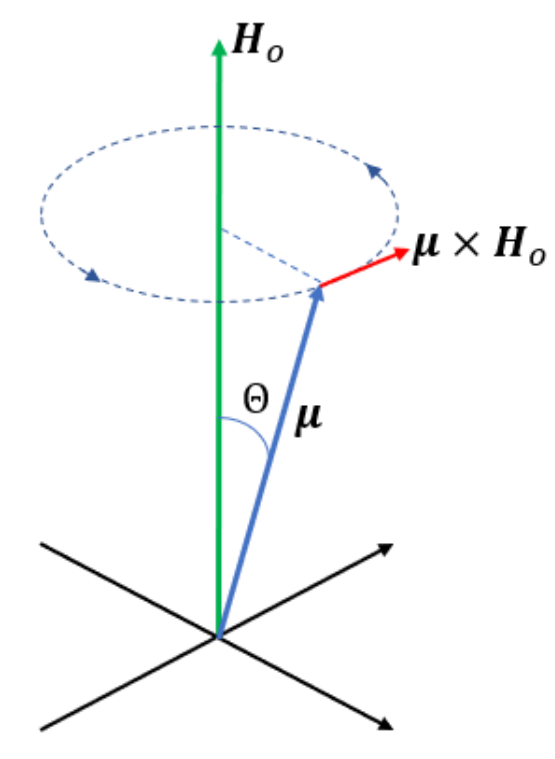

Figure 3.2: Illustration of the precessional cone of cone angle $\Theta$ formed by torque exerted by an external field on a magnetic moment $\boldsymbol{\mu}$.

\subsection{Ferromagnetic Resonance}

Typical ferromagnetic materials are defined by their large exchange fields as well as an internal anisotropic field which enable the magnetic moments to be aligned parallel to each other in the direction of the easy axis of magnetization at equilibrium. This enables one to apply a macrospin description to the ferromagnetic system and define magnetization as:

$$
\boldsymbol{M}=\sum_{V} \frac{\boldsymbol{\mu}}{V}
$$

where $V$ is the sample volume. 
Kittel studied ferromagnetic resonance with shape effects in mind and derives the basic resonance frequencies as he did in case of Nuclear Magnetic Resonance (NMR)[51]. He defined demagnetizing factors $N_{x}, N_{y}, N_{z}$ along the three axes of the specimen such that the relation between internal magnetic field $\left(\boldsymbol{H}_{\text {int }}\right)$ and applied magnetic fields $\left(\boldsymbol{H}_{o}\right)$ are given as:

$$
H_{\text {int }}^{x}=H_{o}^{x}-N_{x} M_{x} ; H_{i n t}^{y}=H_{o}^{y}-N_{y} M_{y} ; H_{i n t}^{z}=H_{o}^{z}-N_{z} M_{z}
$$

For a thin film with applied field along z-direction $\left(M_{x}, M_{y}, M_{z}=M\right), N_{x}=N_{y}=0, N_{z}=1$, thus the equation of motion of spin with macrospin description

$$
\frac{d \boldsymbol{M}}{d t}=\gamma\left(\boldsymbol{M} \times \mu_{\boldsymbol{o}} \boldsymbol{H}_{e f f}\right)
$$

can be decomposed into the following components with $\boldsymbol{B}_{o}=\mu_{o} \boldsymbol{H}_{o}$

$$
\begin{gathered}
\frac{d M_{x}}{d t}=M_{y} \\
\frac{d M_{y}}{d t}=\left[-\gamma\left(B_{o}-\mu_{o} M\right)\right] M_{x} \\
\frac{d M_{z}}{d t}=0
\end{gathered}
$$

For a time-dependent solution $\propto e^{-i \omega t}$, we solve the matrix determinant of the system of equation to get the resonance frequencies,

$$
\omega_{o}=\gamma\left(B_{o}-\mu_{o} M\right)^{\frac{1}{2}}
$$


For the purposes of this thesis, we adopt the free energy picture of this phenomenon where each contributing field is represented by its free energy contribution to calculate the resonances. This is as follows:

The effective field in a ferromagnet is a sum of the applied external field and the internal fields, i.e.,

$$
\boldsymbol{H}_{\text {eff }}=\boldsymbol{H}_{o}+\boldsymbol{H}_{\text {int }}
$$

The other factor affecting magnetization dynamics in ferromagnets is the relaxation or damping process. $\boldsymbol{H}_{e f f}$ in Eqn. (3.8) leads to a precessional motion of magnetic moments in the sample volume which also interacts with local fields, disseminating energy in the process and thus relaxing back to its equilibrium position along $\boldsymbol{H}_{\text {eff }}$. While Eqn. (3.8) describes the precessional motion, the damping factor was added to the equation by Landau-Lifshitz [54] in the form of a phenomenological damping factor $\lambda=\alpha \frac{\gamma}{M}$ in Eqn. (3.8) and summing over the sample volume, to give the Landau-Lifshitz equation for ferromagnetic dynamics:

$$
\frac{d \boldsymbol{M}}{d t}=-\gamma\left[\boldsymbol{M} \times \mu_{\boldsymbol{o}} \boldsymbol{H}_{e f f}\right]-\lambda\left[\boldsymbol{M} \times\left[\boldsymbol{M} \times \mu_{\boldsymbol{o}} \boldsymbol{H}_{e f f}\right]\right]
$$

Here $\alpha$ is a dimensionless quantity called damping factor. The first term in Eqn. (3.14) represents the precessional motion while the second term represents the damping motion and the magnitude of $\boldsymbol{M}$ is preserved. The interaction of these factors once the microwave radiation is turned off is depicted in Figure 3.3. 


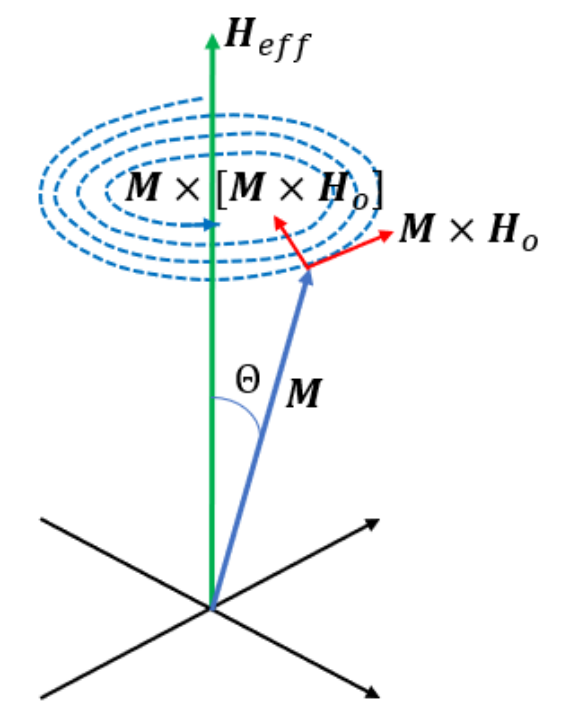

Figure 3.3: When the rf excitation field is turned off, the precessional motion is dampened by damping forces and the magnetization is brought back into equilibrium position before the excitation.

When microwave is applied on the sample, the microwave field $\boldsymbol{h}_{r f}$ perpendicular to the applied field $\boldsymbol{H}_{o}$, the effective field $\boldsymbol{H}_{\text {eff }}$ induces precessional motion in the magnetic moments which get excited by the effective field as well as get relaxed by the damping forces. The resulting effect deflects magnetization $\boldsymbol{M}$ from the equilibrium direction by a cone angle $\Theta$ which ultimately reaches an equilibrium value $\Theta_{o}$ where the damping forces are balanced by the effective field forces. Thus, ferromagnetic resonance condition is met where $\boldsymbol{M}$ precesses with a constant angle $\Theta_{o}$, which is within the small angle regime. This enables us to find the resonance condition using the free energy of the system as proposed by Smit and Suhl. This method has been outlined by Vonsovskii which is given below [55]. 
Since the magnitude of $\boldsymbol{M}$ is conserved, the Landau-Lifshitz equation can be written in spherical coordinates by defining the components of $\boldsymbol{M}$ and $\boldsymbol{H}_{\text {eff }}$ as follows:

$$
\boldsymbol{M}=(M \sin \vartheta \cos \varphi, M \sin \vartheta \sin \varphi, M \cos \vartheta) ; \boldsymbol{H}_{\text {eff }}=\left(H_{M}, H_{\vartheta}, H_{\varphi}\right)
$$

The radial component $H_{M}$, the polar component $H_{\vartheta}$ and the azimuthal component $H_{\varphi}$ of the effective field $\boldsymbol{H}_{\text {eff }}$ are connected with the Cartesian components by the relationships[55]:

$$
\begin{gathered}
H_{M}=H_{x} \sin \vartheta \cos \varphi+H_{y} \sin \vartheta \sin \varphi+H_{z} \cos \vartheta \\
H_{M}=H_{x} \sin \vartheta \cos \varphi+H_{y} \sin \vartheta \sin \varphi+H_{z} \cos \vartheta \\
H_{\varphi}=-H_{x} \sin \varphi+H_{y} \cos \varphi
\end{gathered}
$$

To convert the Landau-Lifshitz equation in Eqn. (3.14) into spherical coordinates, we first define the magnetization vector and its time derivative as well as the effective field in spherical coordinate space as follows:

$$
\begin{gathered}
\boldsymbol{M}=M \hat{e}_{r} \\
\dot{\boldsymbol{M}}=\dot{M} \hat{e}_{r}+M \dot{\vartheta} \hat{e}_{\vartheta}+M \dot{\varphi} \sin \vartheta \hat{e}_{\varphi} \\
\boldsymbol{H}_{e f f}=H_{r} \hat{e}_{r}+H_{\vartheta} \hat{e}_{\vartheta}+H_{\varphi} \hat{e}_{\varphi}
\end{gathered}
$$

This gives us:

$$
\begin{gathered}
\boldsymbol{M} \times \boldsymbol{H}_{e f f}=0 \hat{e}_{r}-M H_{\varphi} \hat{e}_{\vartheta}+M H_{\vartheta} \hat{e}_{\varphi} \\
\boldsymbol{M} \times\left[\boldsymbol{M} \times \boldsymbol{H}_{e f f}\right]=0 \hat{e}_{r}-M^{2} H_{\vartheta} \hat{e}_{\vartheta}+M^{2} H_{\varphi} \hat{e}_{\varphi}
\end{gathered}
$$

Plugging into Eqn. (3.14) gives us the Landau-Lifshitz equation in spherical coordinates: 


$$
\begin{gathered}
\dot{\vartheta}=\gamma \mu_{o}\left(H_{\varphi}+\alpha H_{\vartheta}\right) \\
\dot{\varphi} \sin \vartheta=-\gamma \mu_{o}\left(H_{\vartheta}-\alpha H_{\varphi}\right)
\end{gathered}
$$

The intensity of these field components can be determined by analyzing their relationship with the free energy of the system. At equilibrium, $\boldsymbol{M}$ is oriented along the radial component of $\boldsymbol{H}_{e f f}, H_{M}$.

$$
H_{M}=-\frac{\partial F}{\partial M}
$$

The transverse components $H_{\vartheta}$ and $H_{\varphi}$ vanish in this state and thus the equilibrium orientation angles $\vartheta_{o}$ and $\varphi_{o}$ of the vector $\boldsymbol{M}$ can be determined from the equations:

$$
F_{\vartheta} \equiv \frac{\partial F}{\partial \vartheta}=0 ; F_{\varphi} \equiv \frac{\partial F}{\partial \varphi}=0
$$

whose solutions minimizes the free energy of the system. An arbitrary direction of applied field leads to quite complicated mathematics so we will restrain ourselves to the case where the field is being applied in the direction of easy axis of magnetization.

When microwave radiation is applied on the sample, magnetization is deflected from equilibrium and the transverse fields act as restoring forces. Thus, this non-equilibrium state can be described as a small deviation from equilibrium. The transverse fields take the form:

$$
\mu_{o} H_{\vartheta}=-\frac{F_{\vartheta}}{M} ; \mu_{o} H_{\varphi}=-\frac{F_{\varphi}}{M \sin \vartheta}
$$

The deviations from equilibrium

$$
\delta \vartheta(t)=\vartheta(t)-\vartheta_{o} ; \delta \varphi(t)=\varphi(t)-\varphi_{o}
$$


are small compared to the equilibrium values allowing us to Taylor expand $F_{\vartheta}$ and $F_{\varphi}$ up to linear terms

$$
F_{\vartheta}=F_{\vartheta \vartheta} \delta \vartheta+F_{\vartheta \varphi} \delta \varphi ; F_{\varphi}=F_{\varphi \vartheta} \delta \vartheta+F_{\varphi \varphi} \delta \varphi
$$

where $F_{i j}=\frac{\partial}{\partial i}\left(\frac{\partial F}{\partial j}\right)$ are the second derivatives of the free energy which can be calculated for equilibrium positions. Plugging Eqns.3.23 and 25 back into Eqns.3.19 and 3.20 give us a system of linear equations describing small damped eigen-oscillations of the magnetization vector about the equilibrium position

$$
\begin{gathered}
-\gamma^{-1} M \sin \vartheta_{o} \delta \dot{\vartheta}=\left\{F_{\varphi \vartheta}+\alpha F_{\vartheta \vartheta} \sin \vartheta_{o}\right\} \delta \vartheta+\left\{F_{\varphi \varphi}+\alpha F_{\vartheta \varphi} \sin \vartheta_{o}\right\} \delta \varphi \\
\gamma^{-1} M \sin \vartheta_{o} \delta \dot{\varphi}=\left\{F_{\varphi \varphi}-\alpha F_{\vartheta \varphi}\left(\sin \vartheta_{o}\right)^{-1}\right\} \delta \vartheta+\left\{F_{\varphi \vartheta}-\alpha F_{\vartheta \vartheta}\left(\sin \vartheta_{o}\right)^{-1}\right\} \delta \varphi
\end{gathered}
$$

Harmonic ansatz $\delta \varphi(t) \propto e^{i w t}$ and $\delta \vartheta(t) \propto e^{i w t}$ solve these equations and we get the following resonance eigenfrequency

$$
\omega_{\text {res }}=\gamma\left(1+\alpha^{2}\right)^{\frac{1}{2}} H_{e f f}=\frac{\gamma\left(1+\alpha^{2}\right)^{\frac{1}{2}}}{M \sin \vartheta_{o}}\left\{F_{\vartheta \vartheta} F_{\varphi \varphi}-F_{\vartheta \varphi}^{2}\right\}^{\frac{1}{2}}
$$

The resonant absorption line width is given by

$$
\Delta \omega \equiv\left(\frac{d \omega}{d H}\right) \Delta H=\frac{\gamma \alpha}{M}\left\{F_{\vartheta \vartheta}+F_{\varphi \varphi}\left(\sin ^{2} \vartheta_{o}\right)^{-1}\right\}
$$




\subsection{Antiferromagnetic Resonance}

The resonance problem in antiferromagnets can be extended from ferromagnets as a problem involving two magnetic sublattices interacting with each other via an exchange interaction. The two sublattices have their own magnetization vectors $-\boldsymbol{M}_{1}$ and $\boldsymbol{M}_{2}$, assuming each sublattice acts as a ferromagnetic lattice in itself. This leads to an equation of motion for each magnetization vector interlaced with exchange interaction. The theory of antiferromagnetic resonance (AFMR) has been studied extensively by Kittel and Keffer, Nagamiya, etc.[56-59]. Kittel's equation of motion was extended for a two-sublattice system by Nagamiya, Yoshida and Kubo which is as follows:

Consider a uniaxial antiferromagnet (easy axis along z-direction) with spins on two sublattices. The magnetization vectors $\boldsymbol{M}_{1}$ and $\boldsymbol{M}_{2}\left(\left|\boldsymbol{M}_{1}\right|=\left|\boldsymbol{M}_{2}\right|=M\right)$ of the two sublattices are directed along $+\mathrm{Z}$ and $-\mathrm{z}$ directions respectively (Figure 3.4) due to an anisotropy field $\boldsymbol{B}_{A} \hat{z}$ where $B_{A}=\frac{K}{M}$. The exchange interaction between these two sublattices can be defined as follows:

$$
\begin{aligned}
& \boldsymbol{B}_{E}{ }^{1}=-\lambda \boldsymbol{M}_{2} \\
& \boldsymbol{B}_{E}{ }^{2}=-\lambda \boldsymbol{M}_{1}
\end{aligned}
$$

where $\lambda$ is positive. Thus, the total field acting on the two magnetization vectors are:

$$
\begin{aligned}
& \boldsymbol{B}_{\text {eff }}{ }^{1}=-\lambda \boldsymbol{M}_{2}+B_{A} \hat{\boldsymbol{z}} \\
& \boldsymbol{B}_{\text {eff }}{ }^{2}=-\lambda \boldsymbol{M}_{1}-B_{A} \hat{\boldsymbol{z}}
\end{aligned}
$$




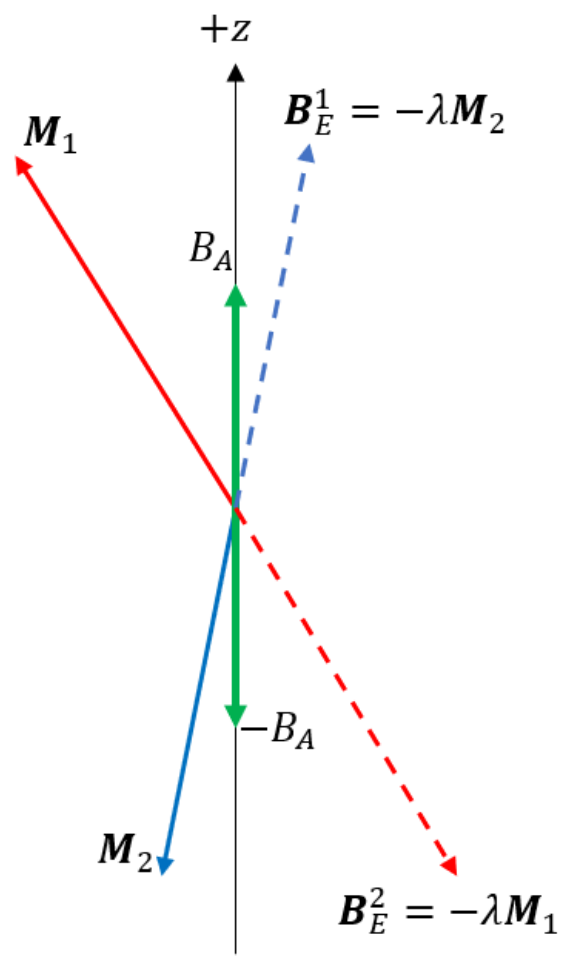

Figure 3.4: Effective fields in the antiferromagnetic resonance of a uniaxial antiferromagnet. Each sublattice magnetization experiences the anisotropy field (acting along the z-axis in this case) and the exchange field due to the other sublattice. Together with the applied field, the effective field interacts with the sublattice magnetizations.

As in the case of ferromagnetic resonance, the equations of motion for the two sublattices:

$$
\begin{aligned}
& \frac{d \boldsymbol{M}_{1}}{d t}=\gamma\left(\boldsymbol{M}_{1} \times \mu_{\boldsymbol{o}} \boldsymbol{H}_{e f f}\right) \\
& \frac{d \boldsymbol{M}_{2}}{d t}=\gamma\left(\boldsymbol{M}_{2} \times \mu_{\boldsymbol{o}} \boldsymbol{H}_{e f f}\right)
\end{aligned}
$$

lead to the following set of linear equations for $M_{1}^{Z}=M$ and $M_{2}^{Z}=-M$ : 


$$
\begin{gathered}
\frac{d M_{1}^{x}}{d t}=\gamma\left[M_{1}^{y}\left(\lambda M+B_{A}\right)-M\left(-\lambda M_{2}^{y}\right)\right] \\
\frac{d M_{1}^{y}}{d t}=\gamma\left[M\left(-\lambda M_{2}^{x}\right)-M_{1}^{x}\left(\lambda M+B_{A}\right)\right] \\
\frac{d M_{2}^{x}}{d t}=\gamma\left[M_{2}^{y}\left(-\lambda M-B_{A}\right)-(-M)\left(-\lambda M_{1}^{y}\right)\right] \\
\frac{d M_{2}^{y}}{d t}=\gamma\left[(-M)\left(-\lambda M_{1}^{y}\right)-M_{2}^{x}\left(-\lambda M-B_{A}\right)\right]
\end{gathered}
$$

Define $M_{1}^{+}=M_{1}^{x}+i M_{1}^{y}$ and $M_{2}^{+}=M_{2}^{x}+i M_{2}^{y}$ such that the Eqns. (3.35-3.38) become

$$
\begin{gathered}
-i \omega M_{1}^{+}=-i \gamma\left[M_{1}^{+}\left(B_{A}+\lambda M\right)+M_{2}^{+}(\lambda M)\right. \\
-i \omega M_{2}^{+}=i \gamma\left[M_{2}^{+}\left(B_{A}+\lambda M\right)+M_{1}^{+}(\lambda M)\right.
\end{gathered}
$$

Solution to these equations can be found by solving the following determinant equation with $B_{E} \equiv$ $\lambda M$

$$
\left|\begin{array}{cc}
\gamma\left(B_{A}+B_{E}\right)-\omega & \gamma B_{E} \\
\gamma B_{E} & \gamma\left(B_{A}+B_{E}\right)+\omega
\end{array}\right|=0
$$

Thus, the antiferromagnetic resonance frequencies are given by

$$
\omega_{o}= \pm \gamma\left[B_{A}\left(B_{A}+2 B_{E}\right)\right]^{\frac{1}{2}}= \pm \gamma \mu_{o}\left[H_{A}\left(H_{A}+2 H_{E}\right)\right]^{\frac{1}{2}}
$$

When an external field is applied on the sample, the resonance frequencies take the form:

$$
\omega_{o}= \pm \gamma \mu_{o} H_{o} \pm \gamma \mu_{o}\left[H_{A}\left(H_{A}+2 H_{E}\right)\right]^{\frac{1}{2}}
$$

The results of Eqn. (3.43) and (3.44) are very interesting. Eqn. (3.43) shows that there is a finite resonance in uniaxial antiferromagnets even when there is no external field which sets AFMR apart from FMR. This finite resonance results from an effective field present due to the exchange and anisotropy fields even in the absence of applied field. 


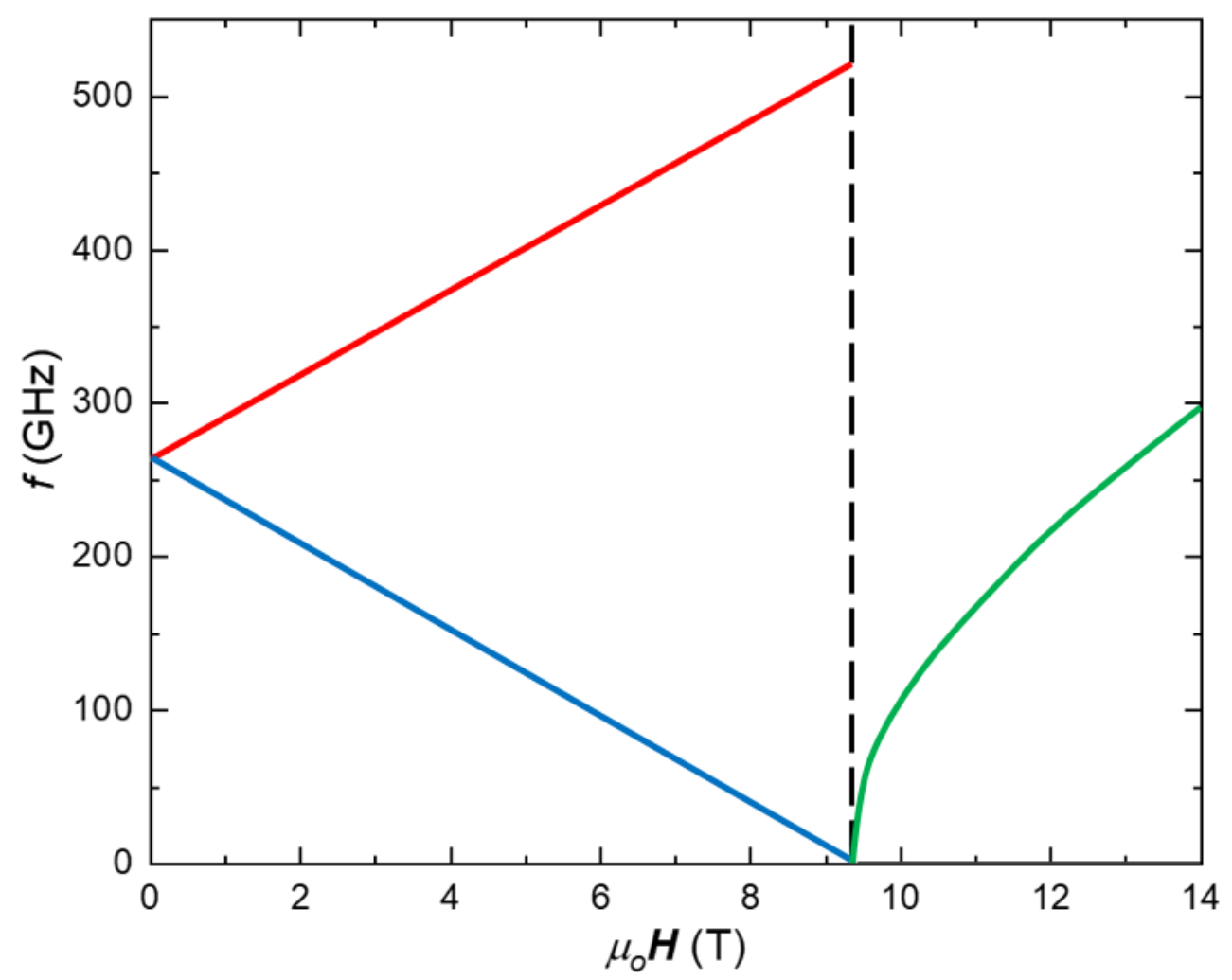

Figure 3.5: Resonance modes calculated by Kittel, Keffer and Nagamiya formalism of AFMR. The red and blue lines represent the Kittel-Keffer [56] described low field resonance mode, the red one being high frequency mode and the blue one being low frequency mode. The green line represent the spin flop mode calculated by Nagamiya [57].

The resonance modes as described by Kittel and Keffer[56] are depicted in Figure 3.5. Two groups of distinct field-dependent modes defined by Eqn. (3.44) are noticed - one that is at a higher frequency $\omega_{H}$ and the other at a lower frequency $\omega_{L}$. At zero field, these modes are degenerate, and the solution is given by Eqn. (3.43). Considering a field applied along positive z-direction, if one looks in the direction of the applied field, one finds that the two magnetization vectors $\boldsymbol{M}_{i}$ 's precess in clockwise direction for one of modes and in counterclockwise direction for the other 
mode. These two modes are degenerate for zero applied field when the two sublattices are identical. When a field $\boldsymbol{H}_{o}$ is applied along the easy axis, the degeneracy is lifted. The frequency of one of the modes $\left(\omega_{H}\right)$ is increased by $\gamma \mu_{o} H_{o}$ while that of the other $\left(\omega_{L}\right)$ is decreased by the same amount as $\boldsymbol{H}_{o}$ is increased. This can be intuitively understood in term of "leading-lagging"like motion. In the $\omega_{H}$ mode, the magnetization $\boldsymbol{M}_{1}$ which is parallel to the applied field $\boldsymbol{H}_{o}$ leads while $\boldsymbol{M}_{2}$ follows the molecular field of $\boldsymbol{M}_{1}$ as its effective field is parallel to the applied field and any increase in the field increases this effective field. Thus, with increasing $\boldsymbol{H}_{o}$ the frequency of $\omega_{H}$ mode also increases. On the other hand, the $\omega_{L}$ sees $\boldsymbol{M}_{2}$ leading while $\boldsymbol{M}_{1}$ follows. But because the effective field of $\boldsymbol{M}_{2}$ is anti-parallel to the applied field, any increase in $\boldsymbol{H}_{\boldsymbol{o}}$ results in overall decrease in the resonance frequency of $\omega_{L}$ mode as shown in Figure 3.5.

The radii of precession of the two magnetization vectors $\boldsymbol{M}_{i}$ 's are not equal unless $H_{A}=0$. Thus, a ratio of the cone angles of the two vectors can be defined as depicted in Figure $3.5: \eta=\theta_{1} / \theta_{2}$ which is greater than 1 for the higher frequency mode $\left(\omega_{H}\right)$ and less than 1 for the lower frequency mode $\left(\omega_{L}\right)$. The value of $\eta$ determines the coupling between the two sublattices [60] and in fact a material dependent parameter given as $: \eta=\frac{\left[H_{A}+H_{E}+\left(H_{A}\left(H_{A}+2 H_{E}\right)\right)^{\frac{1}{2}}\right.}{H_{E}}$. 

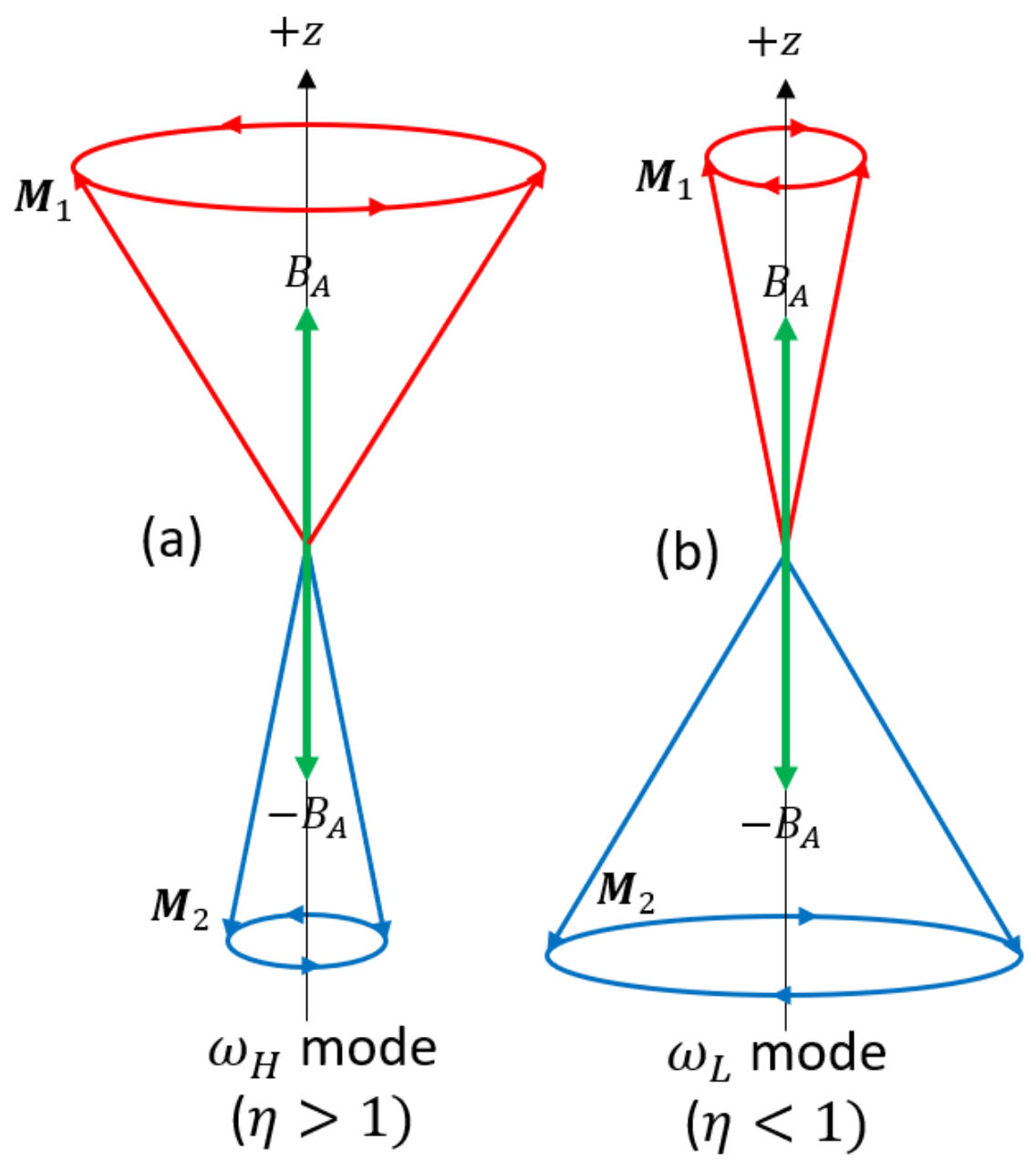

Figure 3.6: Equilibrium orientations of sublattice magnetization at resonance. Two distinct modes exist: A low frequency mode (counter-clockwise) and a high frequency mode (clockwise) defined by the precessional direction. 


\subsubsection{Spin-Flop: Critical Field Resonance}

Treatment of AFMR changes beyond the critical field where the first order phase transition called as Spin-Flop occurs. At this critical field, the two magnetization vectors assume the configuration as depicted below:

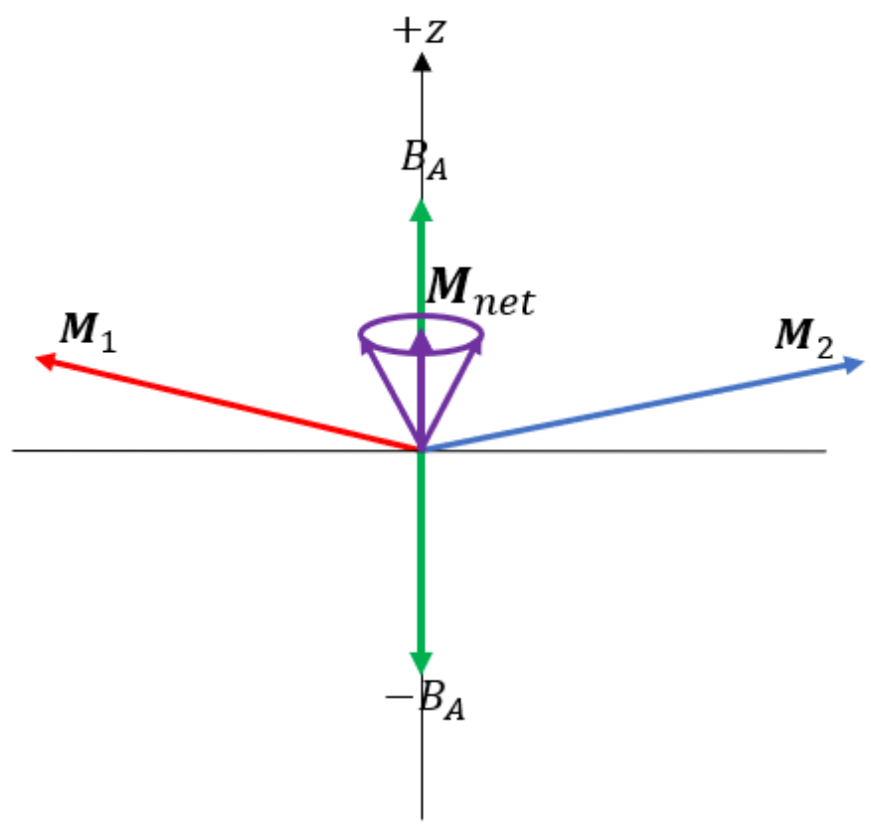

Figure 3.7: Spin Flop Mode where the two magnetizations suddenly become orthogonal as well as canted towards the applied field giving rise to a net magnetization in the canted direction.

The effective field at both the vectors is the same thereby leading to a degeneracy in the vectors with respect to the external field which implies a single resonance line and hence the Kittel conclusion of Eqns. (3.43) and (3.44) do not work here. Nagamiya[59] et.al. have treated this problem and shown that the net magnetization $\left(\boldsymbol{M}_{1}-\boldsymbol{M}_{2}\right)$ induced along the applied field $\boldsymbol{H}_{o}$ after the spin-flop transition displays the same precessional motion as a ferromagnet with the 
magnetization in the same direction, hence aptly called the spin flop mode with quasiferromagnetic nature (SFM). The resonance frequency in this situation is given as:

$$
\omega_{o}= \pm \gamma \mu_{o}\left(H_{o}^{2}-2 H_{E} H_{A}\right)^{\frac{1}{2}}
$$

Hagiwara and co-workers have shown in their landmark experiment the presence of all the resonance modes in $\mathrm{MnF}_{2}$.

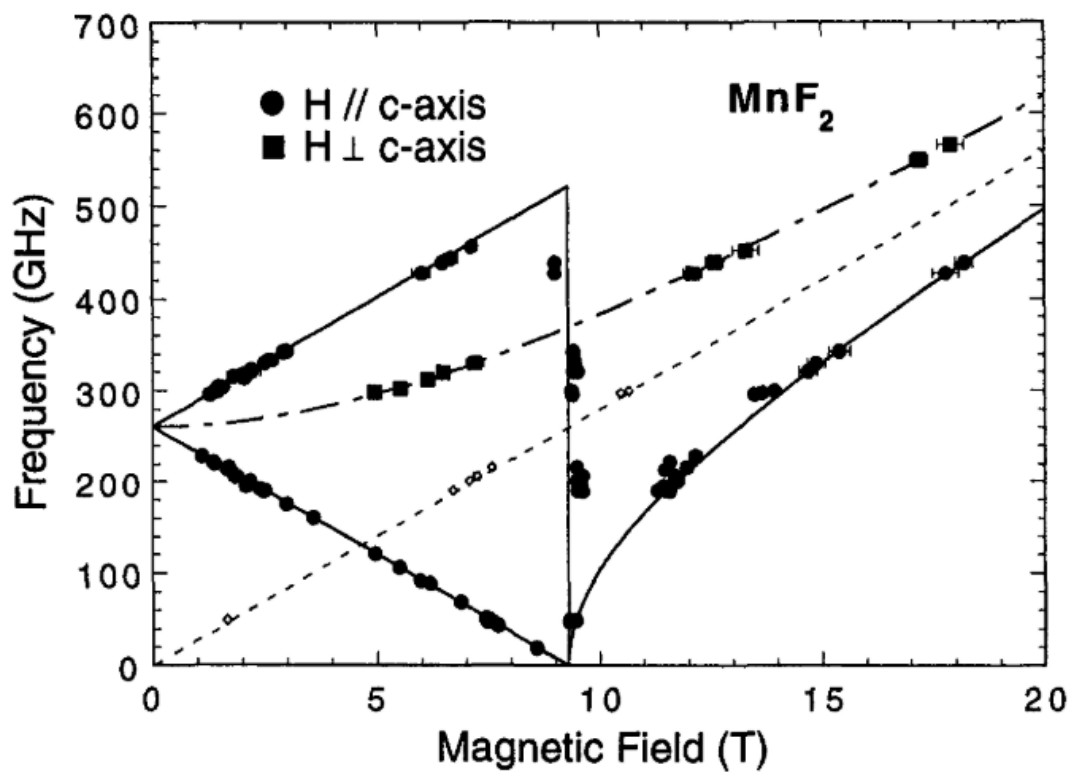

Figure 3.8: Frequency vs external magnetic field relations of the AFMR signals in $M_{n} F_{2}$ as obtained by Hagiwara et. al. [21]. The full lines are theoretical fits for a field applied along the easy axis while the dash-dot lines are theoretical fits for a field applied perpendicular to easy axis.

Figure 3.8 shows the distinct presence of the two branches corresponding to the main AF dynamical modes, the spin-flop transition and the high field spin flop mode. The theories 
predicting these branches are not unified, however. There is no comprehensive analytical theory to this day that can completely explain the low field, transition and high field resonances of uniaxial antiferromagnets.

For the purposes for this thesis, we follow a phenomenological approach to antiferromagnetic resonance quite like the ferromagnetic resonance approach we described earlier. The following section treats this.

\subsection{Phenomenological approach to Antiferromagnetic Resonance}

We begin with the Landau-Lifshitz equations with Gilbert damping in spherical coordinates from Eqns. (3.19) and (3.20). The idea is to find the total free energy of the system for a given magnetic field and minimize it to obtain the equilibrium angles which can then be utilized in the LandauLifshitz equations to find the resonance frequencies. We treat the two sublattices and their magnetization vectors with their own equations giving us:

$$
\begin{gathered}
\dot{\vartheta}_{i}=\gamma \mu_{o}\left(H_{\varphi, i}+\alpha H_{\vartheta, i}\right) \\
\dot{\varphi}_{i} \sin \vartheta_{i}=-\gamma \mu_{o}\left(H_{\vartheta, i}-\alpha H_{\varphi, i}\right)
\end{gathered}
$$

for $i=1,2$. We utilize the relation between the field and its free energy contribution: $\mu_{o} \boldsymbol{H}_{\text {eff }}=$ $-\frac{d F}{d M}$ for each component of effective field as: 


$$
\mu_{o} H_{\vartheta, i}=-\frac{F_{\vartheta_{i}}}{M} ; \mu_{o} H_{\varphi, i}=-\frac{F_{\varphi_{i}}}{M \sin \vartheta_{i o}}
$$

where $F_{x_{i}}=\frac{d F_{x}}{d i}$ for $x=\vartheta, \varphi$ and $i=1,2$. The index "o" in the angle represents equilibrium angle value which can be determined by minimizing the free energy of the system. For a uniaxial antiferromagnet, the chief contributions to free energy comes from Exchange Interaction, Uniaxial Anisotropy and Zeeman Interaction. The first two interactions are explained in more detail in Section 2.3. Thus, the total free-energy of the uniaxial antiferromagnetic system can be written as:

$$
F=F_{e x c}+F_{a n i}+F_{z e e m a n}
$$

where $F_{\text {exc }}=\Lambda \boldsymbol{M}_{1} \cdot \boldsymbol{M}_{2}$ is the free energy term for exchange interaction

$$
\begin{aligned}
& F_{a n i}=K_{\text {uni }}\left[\left(\boldsymbol{c} . \boldsymbol{m}_{1}\right)^{2}+\left(\boldsymbol{c} . \boldsymbol{m}_{2}\right)^{2}\right] \text { is the free energy term for uniaxial anisotropy, and } \\
& F_{\text {Zeeman }}=-\mu_{o} \boldsymbol{H}_{o} \cdot\left(\boldsymbol{M}_{1}+\boldsymbol{M}_{2}\right) \text { is the free energy term for Zeeman interaction }
\end{aligned}
$$

Here $\boldsymbol{c}$ and $\boldsymbol{m}_{i}$ are the unit vectors along the anisotropy axis and the two sublattice magnetizations respectively.

Minimizing Eqn. (3.49) gives the equilibrium angles $\vartheta_{o}, \varphi_{o}$ and $\left.F_{x_{i}}\right|_{\vartheta_{o}, \varphi_{o}}=0$. Then we can perform Taylor expansion of the free energy derivative in all four variables about the equilibrium angles as follows:

$$
\begin{aligned}
& F_{\vartheta_{i}}=F_{\vartheta_{i} \vartheta_{i}} \delta \vartheta_{i}+F_{\vartheta_{i} \varphi_{i}} \delta \varphi_{i}+F_{\vartheta_{i} \vartheta_{j}} \delta \vartheta_{j}+F_{\vartheta_{i} \varphi_{j}} \delta \varphi_{j} \\
& F_{\varphi_{i}}=F_{\varphi_{i} \vartheta_{i}} \delta \vartheta_{i}+F_{\varphi_{i} \varphi_{i}} \delta \varphi_{i}+F_{\varphi_{i} \vartheta_{j}} \delta \vartheta_{j}+F_{\varphi_{i} \varphi_{j}} \delta \varphi_{j}
\end{aligned}
$$

Plugging Eqns. (3.48), (3.50) and (3.51) in Eqns. (3.46) and (3.47) gives us a system of linear differential equations for the motion of the spherical angles of the magnetization vectors: 


$$
\left(\begin{array}{l}
\delta \dot{\vartheta}_{1} \\
\delta \dot{\varphi}_{1} \\
\delta \dot{\vartheta}_{2} \\
\delta \dot{\varphi}_{2}
\end{array}\right)=\aleph(F, M)\left(\begin{array}{l}
\delta \vartheta_{1} \\
\delta \varphi_{1} \\
\delta \vartheta_{2} \\
\delta \varphi_{2}
\end{array}\right)
$$

where $\mathrm{N}(F, M)$ is a matrix containing the second derivatives of $F$ at equilibrium positions. Using harmonic solution to the system of Eqn. (3.52) in the form $\delta \vartheta_{i}=A_{i} e^{i \omega t}$ and $\delta \varphi_{i}=B_{i} e^{i \omega t}$ gives a system of equation of the form :

$$
\left(\begin{array}{cccc}
F_{\vartheta_{1} \varphi_{1}}+A_{1} F_{\vartheta_{1} \vartheta_{1}}+i \omega C_{1} & F_{\varphi_{1} \varphi_{1}}+A_{1} F_{\vartheta_{1} \varphi_{1}} & F_{\varphi_{1} \vartheta_{2}}+A_{1} F_{\vartheta_{1} \vartheta_{2}} & F_{\varphi_{1} \varphi_{2}}+A_{1} F_{\vartheta_{1} \varphi_{2}} \\
F_{\varphi_{1} \vartheta_{2}}-B_{1} F_{\vartheta_{1} \varphi_{1}} & F_{\vartheta_{1} \varphi_{1}}-B_{1} F_{\varphi_{1} \varphi_{1}}-i \omega C_{1} & F_{\vartheta_{1} \vartheta_{2}}-B_{1} F_{\varphi_{1} \vartheta_{2}} & F_{\vartheta_{1} \varphi_{2}}-B_{1} F_{\varphi_{1} \varphi_{2}} \\
F_{\vartheta_{1} \varphi_{2}}+A_{2} F_{\vartheta_{1} \vartheta_{2}} & F_{\varphi_{1} \varphi_{2}}+A_{2} F_{\varphi_{1} \vartheta_{2}} & F_{\vartheta_{2} \varphi_{2}}+A_{2} F_{\vartheta_{1}} \vartheta_{2}+i \omega C_{2} & F_{\varphi_{2} \varphi_{2}}+A_{2} F_{\vartheta_{1} \varphi_{2}} \\
F_{\vartheta_{1} \vartheta_{2}}-B_{2} F_{\vartheta_{1} \varphi_{2}} & F_{\varphi_{1} \vartheta_{2}}-B_{2} F_{\varphi_{1} \varphi_{2}} & F_{\vartheta_{2} \vartheta_{2}}-B_{2} F_{\vartheta_{2} \varphi_{2}} & F_{\vartheta_{2} \varphi_{2}}-B_{2} F_{\varphi_{2} \varphi_{2}}-i \omega C_{2}
\end{array}\right)=0
$$

where $A_{i}=\alpha \sin \vartheta_{i o}, B_{i}=\frac{\alpha}{\sin \vartheta_{i o}}$ and $C_{i}=\frac{1}{\gamma M \sin \vartheta_{i o}}$ for $i=1,2$.

Solving Eqn. (3.53) yields the four eigenfrequencies. We will be utilizing this matrix equation in Matlab® to fit our data and the details of the fitting are given in Section 5.6. The effect of misalignment can also be studied from Eqn. (3.53) by introducing the projection of the applied field $\boldsymbol{H}_{o}$ along the direction of anisotropy axis. This formalism has been used to fit the AFMR data in Chapter 5.

The precessional motion for the two sublattice magnetizations for perfect alignment of applied field along the anisotropy axis in the different regimes of AFMR can be illustrated in Figure 3.9. The nature of this precession can be extracted from the eigencoefficients of Eqn. (3.52). The calculated AFMR spectra for perfect alignment of applied field and anisotropy axis shown in Figure 3.9 is based on the values given in Table 5.2. 


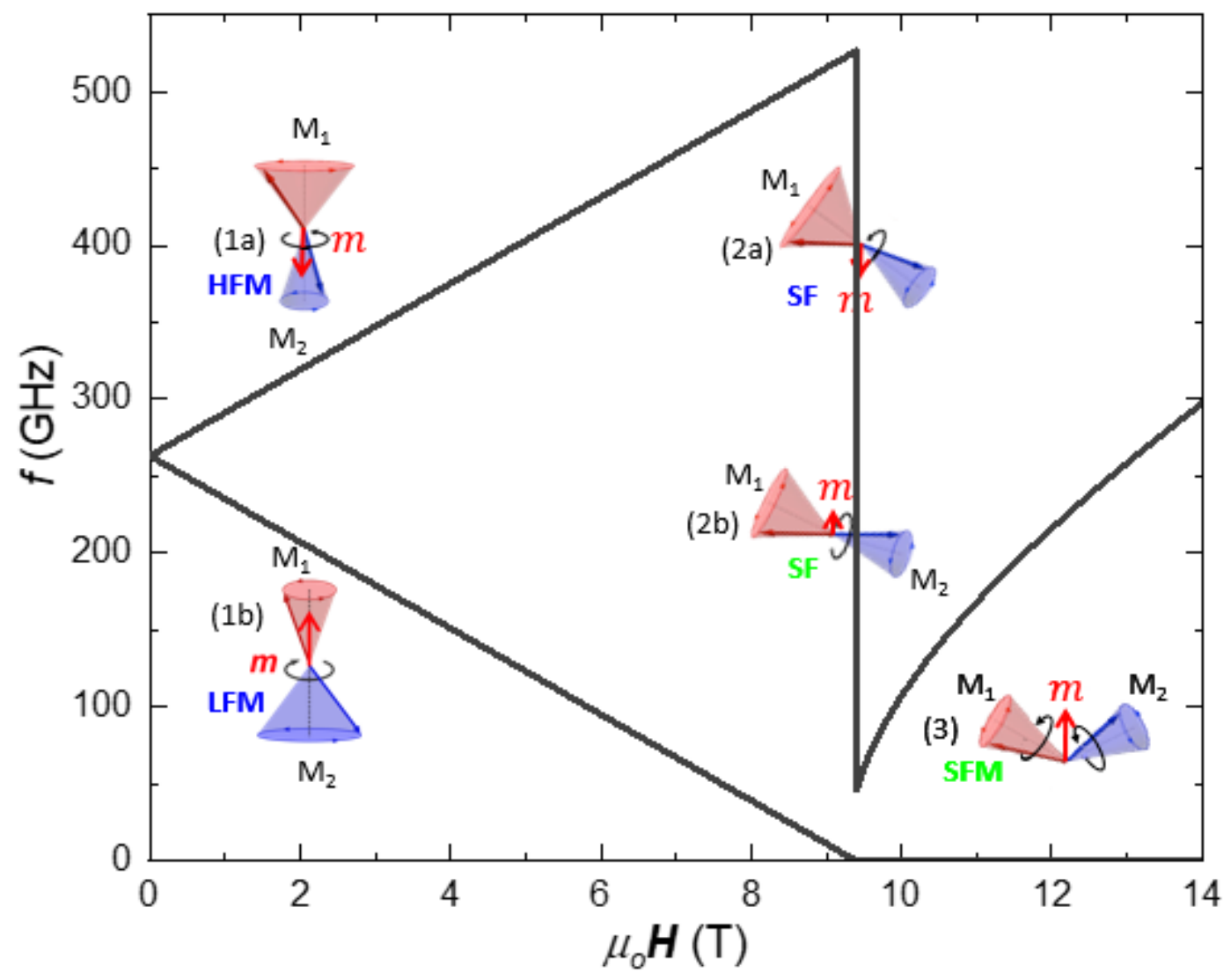

Figure 3.9: Nature of precessional cones of $\boldsymbol{M}_{1}$ and $\boldsymbol{M}_{2}$ in (1) the low field (a) high frequency (b) low frequency, $(2 a / b)$ spin flop transition and (3) spin flop mode. 


\section{CHAPTER 4 : SPINTRONICS THEORY}

This chapter provides an introduction to spintronics and spintronic applications central to this thesis.

\subsection{Introduction}

An electron has two fundamental properties: "charge" and "spin", among others. Charge of an electron has been studied and manipulated extensively, both in academia and industry. In fact, the entire field of electronics is based on the detection and manipulation of electronic charge. On the other hand, magnetism originates from the other fundamental property of electrons which is spin. Individual atoms possess local magnetic moments due to uncompensated electron spins and an exchange coupling between the magnetic moments of neighboring atoms lead to long range ordering in magnetic materials at low temperatures. Ferromagnetism results when the sign of the coupling is positive, and the magnetic moments are all aligned parallel to each other. Similarly, when the sign of the coupling is negative and the magnetic moments are anti-parallel to each other, antiferromagnetism originates. For the longest time, charge and spin of electrons were investigated separately and a correlation between them was not paid much attention[61].

When magnetoresistance was discovered by Lord Kelvin in 1856, a dependency of conductivity on magnetic field, particularly its relative direction, was found. Then much later in 1986, Grunberg et. al.[62] studied $\mathrm{Fe} / \mathrm{Cr} / \mathrm{Fe}$ heterostructures and found that the resistance between the two $\mathrm{Fe}$ layers separated by an antiferromagnetic spacer layer of $\mathrm{Cr}$ was larger when the two layers were 
spontaneously aligned anti-parallel to each other and was lower when the two layers were aligned parallel by a sufficiently large magnetic field. This discovery was further studied and confirmed by Fert et.al.[63] in 1988 and thus Giant Magnetoresistance (GMR) was discovered. For their effort, Grunberg and Fert were jointly awarded the Nobel Prize in Physics in 2007. The discovery of GMR led to the advent of spintronics which encompasses theories and application pertaining to the spin property of electrons.

The spin of an electron is an intrinsic form of angular momentum that the electron possesses. In fact, such intrinsic angular momentum or spin is carried by most of the elementary particles such as hadrons and atomic nucleus. In fact, nuclear spin is extensively used in NMR studies and medical applications. Famously observed in the Stern-Gerlach experiment, the idea of electronic spin was first put forth by Wolfgang Pauli in 1924 in the form of a two valued non classical "hidden rotation" which he later developed a full theory on in 1927[64]. Paul Dirac also used electron spin in his relativistic quantum mechanics theory in 1928. In fact, the spin and magnetic moment of the electron arise naturally from the Dirac equation[65] as $\hbar / 2$ and $e \hbar / 2 m_{e}$.

In the rest of this section we will represent the spin of an electron as $S$ which can be detected by its magnetic moment $-g \mu_{B} \boldsymbol{S}$, where $\mu_{B}$ is the Bohr magneton and $g$ is the electron $\mathrm{g}$ factor. The goal of spintronics is to understand how electronic spin interacts with its solid state environment, thereby leading to development of useful spin-based devices. It has many advantages over electronics, particularly the fact that electronic technology is based on electron motion which is 
susceptible to low efficiency due to scattering while spintronics is based on transfer of angular momentum between charges which can not only be faster but also is more efficient in terms of heat dissipation and power consumption. In its attempt to abide by Moore's law, which is reaching its limit with semiconductor based devices, current technology could find spintronics as a suitable alternative as it allows for compact devices with faster operations to be made out of common materials such as $\mathrm{Fe}, \mathrm{Cu}$ and $\mathrm{Ag}$.

The following sections give an overview of some of the important concepts in spintronics that pertains to the scope of this thesis.

\subsection{Spin Hall Effect and Inverse Spin Hall Effect}

Spin Hall Effect (SHE) is an analogous phenomenon to Hall Effect in electronics. First observed in mid 2000s [66], SHE is now a widely used method to generate and control spin currents. SHE originates from the coupling of the charge and spin currents due to spin-orbit interaction [67]. In direct SHE (Figure 4.1a), a charge current passing through a material with relativistic spin-orbit coupling can generate a transverse pure spin-current polarized perpendicular to the plane defined by the charge and spin current [66]. It has a reciprocal effect, the Inverse Spin Hall Effect (ISHE) (Figure 4.1b) where a pure spin-current through a material generates a transverse charge current. Thus, one may infer SHE and ISHE to be a standard mechanism for spin-charge interconversion. The efficiency of conversion can be studied by defining a material dependent parameter: the spin 
Hall angle $\left(\theta_{S H}\right)$, which is defined as the ratio of spin current to charge current $\left(\left|J_{S}\right| /\left|J_{C}\right|\right)$ such that $J_{S}=\theta_{S H}\left(\hat{\sigma} \times J_{C}\right)$, where $\hbar J_{S} / 2 e, J_{C}$ and $\hat{\sigma}$ are the spin current density, charge current density and spin moment respectively.

(a)

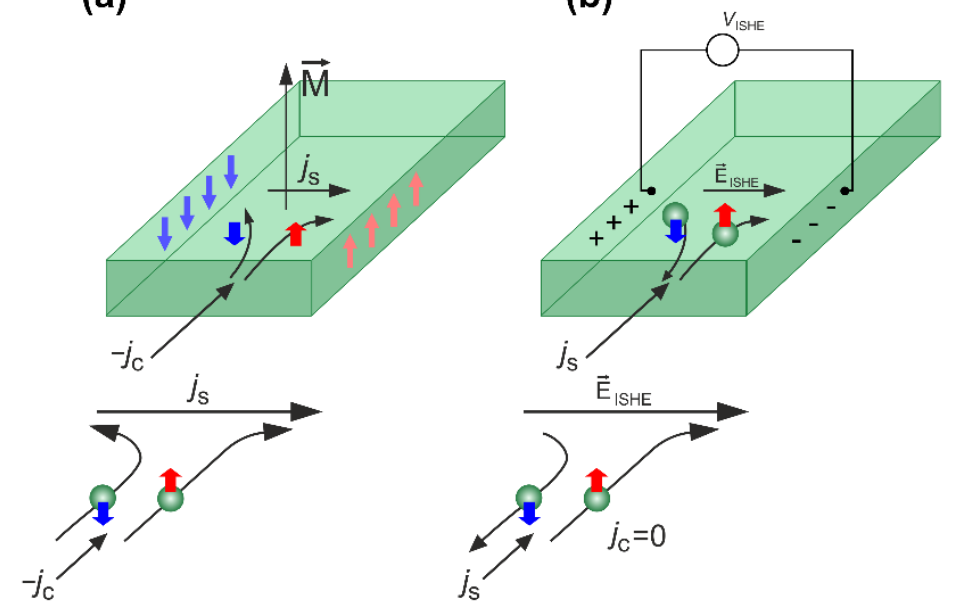

Figure 4.1: Illustration of (a) spin Hall effect and (b) inverse spin Hall effect

\subsection{Spin Pumping and Spin Transfer Torque}

Spin pumping is a method of generating spin current where a magnetic system is driven into resonance using microwaves and external magnetic field and in the resulting precessional motion, the magnetization vector transfers spin angular momentum into an adjacent non-magnetic material (e.g., Pt or W), where the injected spin can be detected via ISHE. Its reciprocal effect is the spin transfer torque effect, when a spin current exerts a torque on a magnetic system (e.g., ferromagnet) 
inducing spin dynamics. Experimentally, spin pumping phenomenon was first observed in early 2000's [66] where experiments showed an enhanced Gilbert damping in FMR associated with the loss of angular momentum by a spin-current flowing from a ferromagnet to a non-magnetic metal. The theoretical framework for spin pumping was presented by Y. Tserkovnyak and A. Brataas in $2002[68,69]$.

\subsubsection{Spin Pumping in a Ferromagnet}

Tserkovnyak et. al. derived the adiabatic spin pumping theory from a scattering formalism as an extension of adiabatic quantum pumping by incorporating spin degrees of freedom [69]. When a magnetic field is applied to a ferromagnetic/non-magnetic metal (F/NM) bilayer, magnetization precession in the ferromagnet gives rise to a time-dependent spin-current at the F/NM interface that flows into the NM layer given by,

$$
I_{S}^{S P}=\frac{\hbar}{4 \pi}\left(A_{r} \boldsymbol{m} \times \frac{d \boldsymbol{m}}{d t}-A_{i} \frac{d \boldsymbol{m}}{d t}\right)
$$

where $A \equiv A_{r}+i A_{i}$ is the complex spin-pumping conductance parameter and $\boldsymbol{m}(t)$ is the unit vector for the magnetization of the ferromagnetic layer. This conductance parameter can be expressed in terms of transmission and reflection coefficients as:

$$
A=g^{\uparrow \downarrow}-t^{\uparrow \downarrow}
$$

where $g^{\uparrow \downarrow}$ is the complex interfacial mixing conductance and $t^{\uparrow \downarrow}$ is the transmission matrix. For ferromagnetic films that are thicker than their spin-coherence length, spin pumping through the 
interface is only governed by $g^{\uparrow \downarrow}$. Furthermore, $A_{i}$ is found to be negligible for most F/NM layers and thus from Eqns. (4.1) and (4.2), we arrive at the pumped spin-current:

$$
I_{S}^{S P}=\frac{\hbar}{4 \pi} g_{r}^{\uparrow \downarrow} m \times \frac{d m}{d t}
$$

where $g_{r}^{\uparrow \downarrow}$ is the real part of the mixing conductance.

To get a complete picture of the kinds of currents involved, one has to consider the backflow of spin current $\left(I_{S}^{B}\right)$ into the $\mathrm{F}$ layer as well. Thus, the total spin accumulation can be written as:

$$
I_{S}=I_{S}^{S P}-I_{S}^{B}
$$

The illustration of these current can be seen in Figure 4.2.

However, for highly conductive NM layers, backscattering is minimal and thus one can approximate $I_{S}^{B} \approx 0$. This leads to the total spin current transferred across the F/NM interface:

$$
I_{S}=\frac{\hbar}{4 \pi} g_{r}^{\uparrow \downarrow} m \times \frac{d m}{d t}
$$

In real systems, spins undergo relaxation over the spin diffusion length of the NM layer and so pure spin currents are not conserved. 


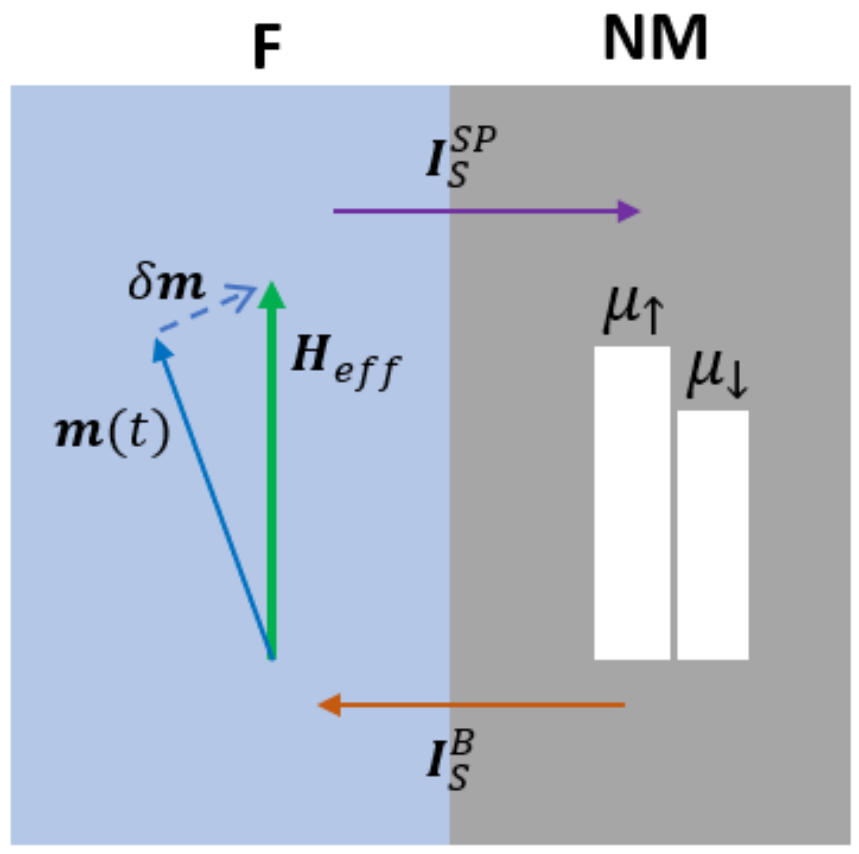

Figure 4.2: Illustration of the two types of current at the F/NM interface. The spin current pumped due to precessing magnetic moment in effective field diffuses along the spin diffusion length in the NM layer. The current due to backscattering is minimal for very conductive NM layer.

\subsubsection{Spin Pumping in Antiferromagnets}

Spin pumping in antiferromagnets has been a difficult topic of study in the past, primarily due to the fact that spin pumping is generated from the precessing magnetization in magnetic materials and in the case of antiferromagnets, the magnetization $\boldsymbol{m}$ is zero at equilibrium and quite small even when the AF system is driven out of equilibrium. As a result, one could presume that the spin currents pumped by the two sublattices will be equal and opposite and thus cancel each other out, 
making the detection of spin pumping impossible. However, Ran Cheng and Arne Brataas have shown this not to be the case, as they show in their report published in 2014 [45]. For a uniaxial, two-sublattice AF material, a general way of interpreting the system is to visualize two independent $\mathrm{F}$ subsystems with defined magnetic moment vectors $\boldsymbol{m}_{1}$ and $\boldsymbol{m}_{2}$. The spin currents pumping from them will be proportional to $\boldsymbol{m}_{1} \times \dot{\boldsymbol{m}}_{1}$ and $\boldsymbol{m}_{2} \times \dot{\boldsymbol{m}}_{2}$ respectively.

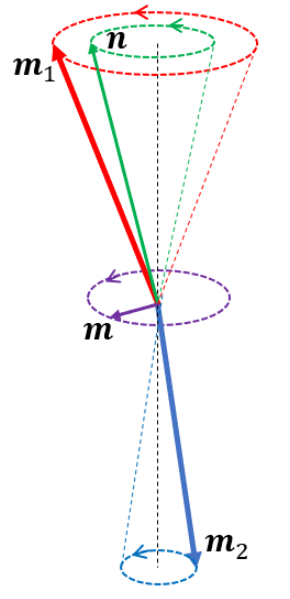

Right-Handed Mode

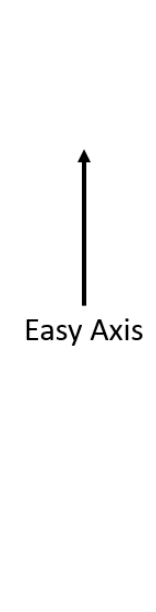

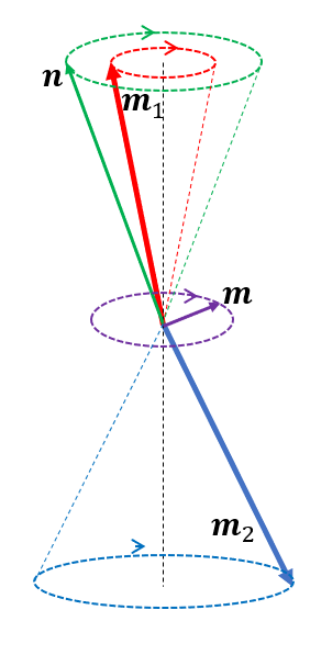

Left-Handed Mode

Figure 4.3: The eigenmodes associated with AFMR that show opposite chiralities. The illustration also shows how the staggered magnetization and the small net magnetization move with reference to the two magnetizations in each mode.

From Figure 4.3, one can infer that $\boldsymbol{m}_{1} \approx-\boldsymbol{m}_{2}$ and $\dot{\boldsymbol{m}}_{1} \approx-\dot{\boldsymbol{m}}_{2}$, thus leading to the conclusion that the contributions from the two subsystems are the same and add up constructively. As a result, one may use the staggered magnetization $\boldsymbol{n}=\left(\boldsymbol{m}_{\mathbf{1}}-\boldsymbol{m}_{2}\right) / 2$ to find that the total spin current is 
proportional to $\boldsymbol{n} \times \dot{\boldsymbol{n}}$. One should not ignore the fact that the different cone angles for the two magnetizations in each mode leads to a small magnetization $\boldsymbol{m}$. Moreover, the mixing of different scattering channels at the $\mathrm{AF} / \mathrm{NM}$ interface prevents an $\mathrm{AF}$ system from being equivalent to two ferromagnets.

Cheng et.al. compared the $\mathrm{THz}$ resonance frequency of antiferromagnets with the energy scales of Fermi energy and exchange coupling to find that the motion of staggered magnetization is essentially adiabatic. The two vectors $\boldsymbol{m}$ and $\boldsymbol{n}$ are treated as two independent adiabatic parameters in a scattering picture to get the following pumped spin current [45]:

$$
\frac{e}{\hbar} I_{s}=G_{r}(\boldsymbol{n} \times \dot{\boldsymbol{n}}+\boldsymbol{m} \times \dot{\boldsymbol{m}})-G_{i} \dot{\boldsymbol{m}}
$$

Here $I_{S}$ is measured in the units of electrical current. The spin mixing conductance $G_{r}$ and $G_{i}$ are different than that of FMs resulting from the mixing of scattering channels from different sublattices. Since the dynamics of AF systems is much faster than ferromagnets, the spin pumping signal is expected to be stronger.

Time averaging Eqn. (4.6) gives rise to the dc component of spin current $I_{S}^{d c}$ with the second term in Eqn. (4.6) vanishing. When a circularly polarized microwave with oscillating field $h_{a c}$ perpendicular to easy axis is applied, AF dynamics is set into motion and the mode from Figure 4.3 that matches the polarization will be driven into resonance at a certain frequency. When the applied field disappears, the $I_{s}^{d c}$ becomes an odd function of microwave frequency $\omega$ which leads 
one to conclude that the direction of dc spin current is dependent on the circular polarization of the microwaves. In addition to the dc spin current, one may also define a staggered spin current which represents the imbalance between the spin current carried by the two sublattices. The dc component of this staggered spin current $I_{s S}^{d c}$ is an even function of $\omega$. The two currents as a function $\omega$ is shown in Figure 4.4.

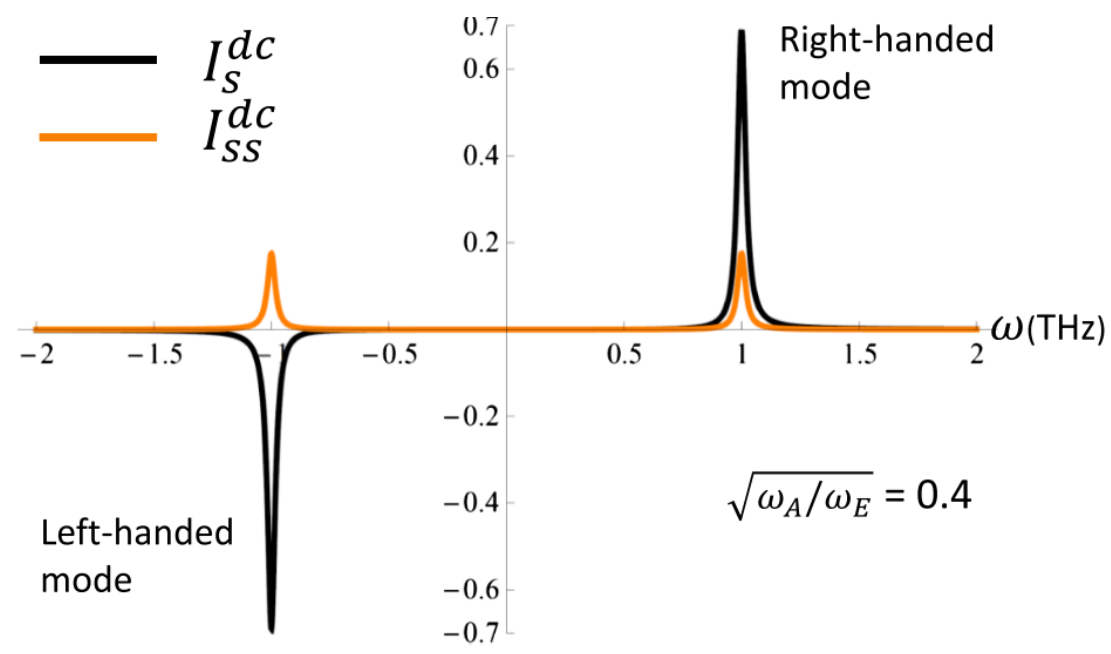

Figure 4.4: DC components of pumped spin current (black) and staggered spin current (orange) as functions of $\omega$ in units of $\left(\frac{\hbar}{e}\right) G_{r}\left(\gamma h_{a c}\right)^{2}$ as calculated by Cheng et.al. for static field frequency $\omega_{H}=0$, resonance frequency $\omega_{R}=1 T H z$ and Gilbert damping $\alpha=0.01 .[45]$

Cheng et.al. advise that the efficiency of microwave absorption at resonance point in an $\mathrm{AF}$ is proportional to $\sqrt{\omega_{A} / \omega_{E}}$ and attribute the failure to observe ISHE signals generated from spin pumping in $\mathrm{MnF}_{2}$ in [46] to a low ratio of $\sqrt{\omega_{A} / \omega_{E}}$. Figure 4.5 shows the resonance value of $I_{S}^{d c}$ 
plotted against different values of $\sqrt{\omega_{A} / \omega_{E}}$, calculated by Cheng et. al. On the basis of this plot, the authors recommend $\mathrm{FeF}_{2}$ to be a better candidate for spin pumping measurements than $\mathrm{MnF}_{2}$ as $\mathrm{FeF}_{2}$ has a larger ratio of $\sqrt{\omega_{A} / \omega_{E}}$.

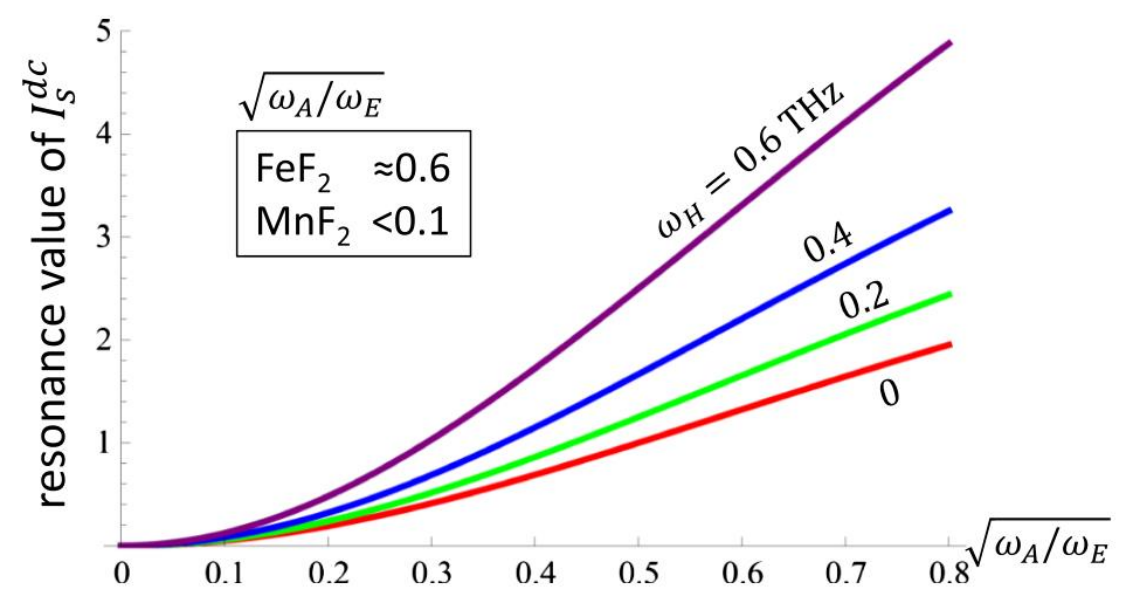

Figure 4.5: For a fixed value of microwave power, the resonance value of $I_{S}^{d c}$ scales with $\sqrt{\omega_{A} / \omega_{E}}$ which presents $\mathrm{FeF}_{2}$ with $\sqrt{\omega_{A} / \omega_{E}} \approx 0.6$ to be a better candidate for spin pumping than $\mathrm{MnF}_{2}$ with $\sqrt{\omega_{A} / \omega_{E}}<0.1 .[45]$

Øyvind et.al. in their 2017 paper [46] added upon the results obtained by Cheng et.al. The pumped spin current from a dynamical AF mode given by Eqn. (4.6) leads to spin accumulation in the NM layer, which in turn produces a spin backflow current. In AF insulators particularly, the backflow spin currents within the sublattices add up constructively to give:

$$
\boldsymbol{I}_{s}^{B}=-\frac{G_{r}}{2 \pi}\left(\boldsymbol{m} \times\left(\boldsymbol{\mu}_{s}^{N} \times \boldsymbol{m}\right)+\boldsymbol{n} \times\left(\boldsymbol{\mu}_{s}^{N} \times \boldsymbol{n}\right)\right)
$$


where $\boldsymbol{\mu}_{s}^{N}$ is the spin accumulation in the normal metal. This spin accumulation is a solution to the spin diffusion equation [46]:

$$
\frac{\partial \boldsymbol{\mu}_{s}^{N}(\boldsymbol{r}, t)}{\partial t}=\gamma_{N} \boldsymbol{H}_{e x} \times \boldsymbol{\mu}_{s}^{N}+D_{N} \frac{\partial^{2} \boldsymbol{\mu}_{s}^{N}}{\partial y^{2}}-\frac{\boldsymbol{\mu}_{s}^{N}}{\tau_{s f}^{N}}
$$

where $D_{N}$ is the diffusion coefficient of NM, $\gamma_{N}$ is the gyromagnetic ratio, $\tau_{s f}^{N}$ is the spin-flip relaxation time and $\boldsymbol{H}_{e x}$ is the external magnetic field. The directions of the two spin currents are illustrated in Figure 6.

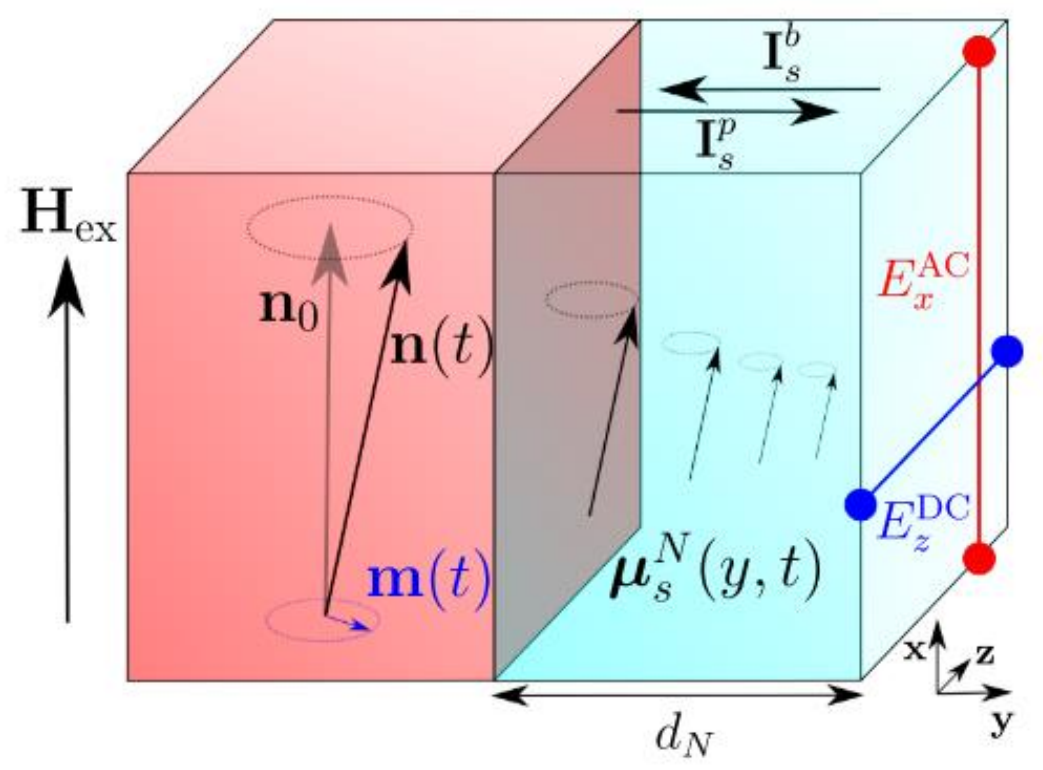

Figure 4.6: Illustration by Øyvind et. al. of precession of $\boldsymbol{m}$ and $\boldsymbol{n}$ in the AF that results in spins being pumped $\left(\boldsymbol{I}_{S}^{p}\right)$ into the NM. The resulting spin accumulation $\boldsymbol{\mu}_{s}^{N}$ in NM causes a backflow $\left(\boldsymbol{I}_{S}^{b}\right)$ of spins back into the AF. [46] 
The ISHE current generated in the normal metal due to spin pumping across the AF/NM interface is given by $[70,71]$ :

$$
\boldsymbol{J}_{C}^{I S H E}(y, t)=\theta_{S H} \frac{2 e}{A \hbar} \boldsymbol{e}_{y} \times \boldsymbol{I}_{s}^{N}(y, t)
$$

where $\mathrm{A}$ is the area of the AF/NM interface. This charge current accumulates charges at the interfaces as the AF is insulating. An electric field is generated which causes the net charge current through the metal to vanish [46]. The DC and first harmonic AC components of this ISHE electric field for $\mathrm{MnF}_{2}$ is calculated by Øyvind et.al. and shown in Figure 4.7.

Figure 4.7 shows some interesting results. In both $\mathrm{FeF}_{2}$ and $\mathrm{MnF}_{2}$, there is a contribution in $\mathrm{DC}$ signal that is independent of AC field polarization and another contribution that is proportional to $\sin \left(\theta_{z}-\theta_{y}\right)$. This polarization dependent contribution can be explored in both of these materials at low fields with suitable frequencies in future work and could possibly lead to interesting physics. 

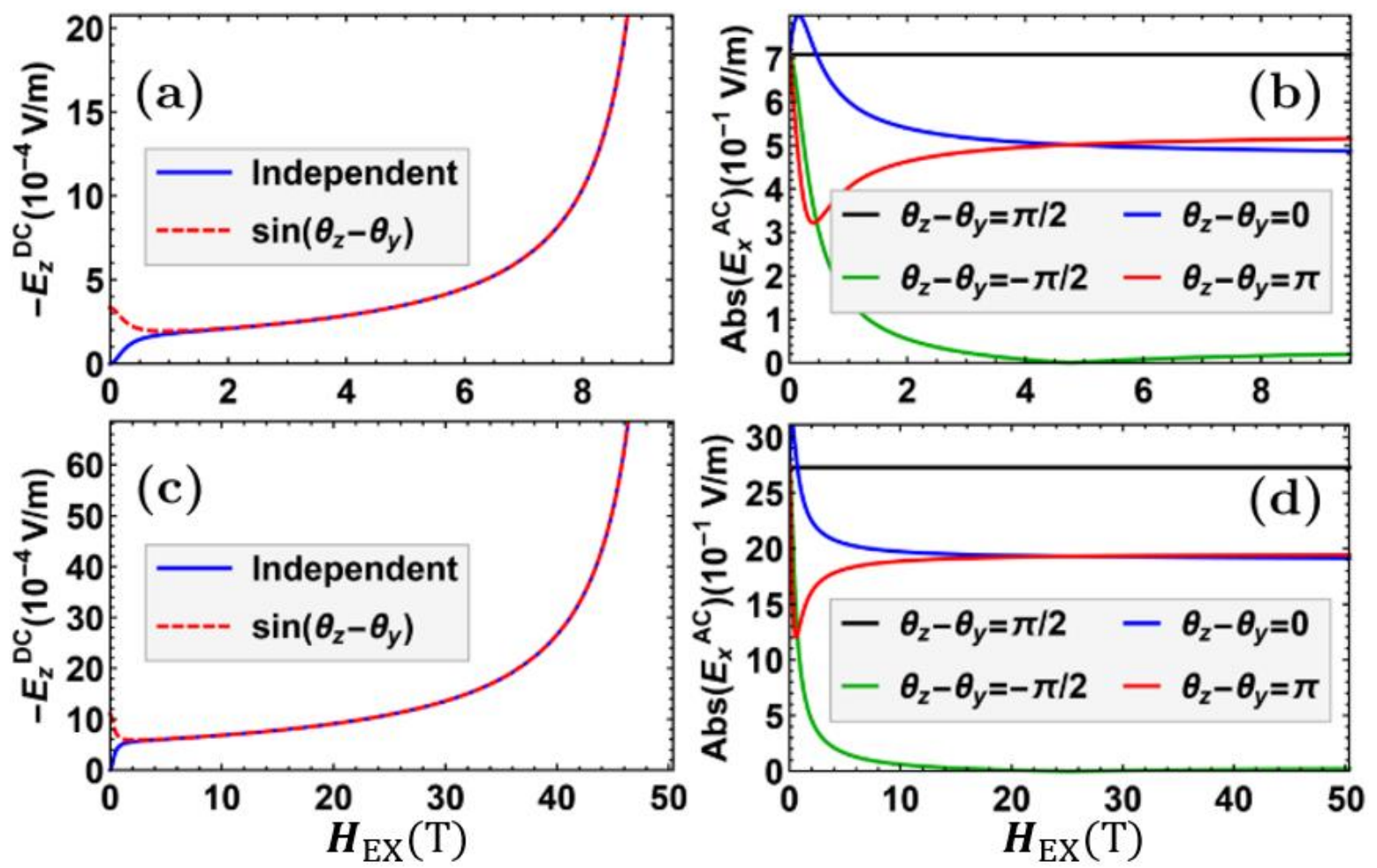

Figure 4.7: Calculated (a/c) DC component (b/d) first harmonic AC component of the ISHE electric field as a function of external magnetic field along the easy axis of $\mathrm{MnF}_{2}(a, b)$ and $\mathrm{FeF}_{2}(c$, d). $(b, d)$ shows different polarizations of the AC magnetic field. The AC field is $\operatorname{ImT}$ and $\alpha=$ $0.01[46]$.

Øyvind et.al advised that the divergence of the DC signal observed near the spin-flop field for both materials can be utilized to enhance the ISHE signal which is perhaps more realistically achieved in $\mathrm{MnF}_{2}(\sim 9.5 \mathrm{~T})$ than in $\mathrm{FeF}_{2}(\sim 50.4 \mathrm{~T})$ due to really high spin-flop field value in the later. In addition to this, a larger DC signal is achieved with circular polarization of the AC field $\left(\theta_{z}-\right.$ $\left.\theta_{y}=\pi / 2\right)$ 


\subsection{Spin Seebeck Effect}

Just like spin pumping, spin Seebeck effect (SSE) is another mechanism of spin current generation where thermal gradient in a magnetic material creates a spin-related voltage that can be detected via electrical measurements. But unlike coherent spin pumping due to precessing magnetization, SSE is a thermal magnon dominated process based on scattering [72]. Recent observations [7376] have established SSE as a prominent method of spin current injection, particularly for insulating ferromagnets $[77,78]$. In case of a ferromagnetic insulator (FI), conduction electrons from NM undergo scattering at the FI/NM interface during which, they reverse their spin state by emitting or absorbing magnons in the FI as shown in Figure 4.8 [72]. This magnon excitation gives rise to spin angular momentum transfer between the FI and NM layers. When the temperature of the FI and NM layers are equal, the scattering from both sides of the interface (as shown in Figure 4.8 (a) and (b)) are balanced and no spin current flows across the interface. But in the presence of a temperature gradient, one of these processes dominates over the other and spin current flows across the interface.

In recent times, SSE has been observed in antiferromagnets as well, particularly in antiferromagnetic insulators (AFI) $[16,17,79,80]$. This has led to a lot of research into the origin of SSE in AFI-based heterostructures. $\mathrm{In}_{2} \mathrm{Cr}_{2}$, the SSE signal is observed substantially only after the applied external field exceeds the spin-flop transition field of $\mathrm{Cr}_{2} \mathrm{O}_{3}$ and the signal is found to be proportional to the net equilibrium magnetization of $\mathrm{Cr}_{2} \mathrm{O}_{3}$ [17] which leads one to conclude that field-induced magnetic moment is the source of SSE. But in another experiment on a $\mathrm{MnF}_{2} / \mathrm{Pt}$ 
heterostructure [16], a finite SSE signal was observed even at fields lower than the spin-flop transition showing that there might be another source of SSE at lower fields. While in FI/NM heterostructures, below the Curie temperature, a temperature gradient driven magnon flow is the source of spin current in SSE, in uniaxial AFI/NM heterostructures, the picture is slightly different. Two magnon modes with opposite chiralities and opposite angular momenta exist in most uniaxial AFI such as $\mathrm{MnF}_{2}$ and $\mathrm{FeF}_{2}$. These modes are degenerate at zero field and hence no net magnon flow occurs. Rezende et.al. [18] propose that a field-induced AF magnon imbalance can lead to a characteristic SSE peak even when there is no net equilibrium magnetization which explains the low-field SSE signal observed in $\mathrm{MnF}_{2} / \mathrm{Pt}$ [16]. Thus, a unified picture of SSE in AFI/NM is lacking.
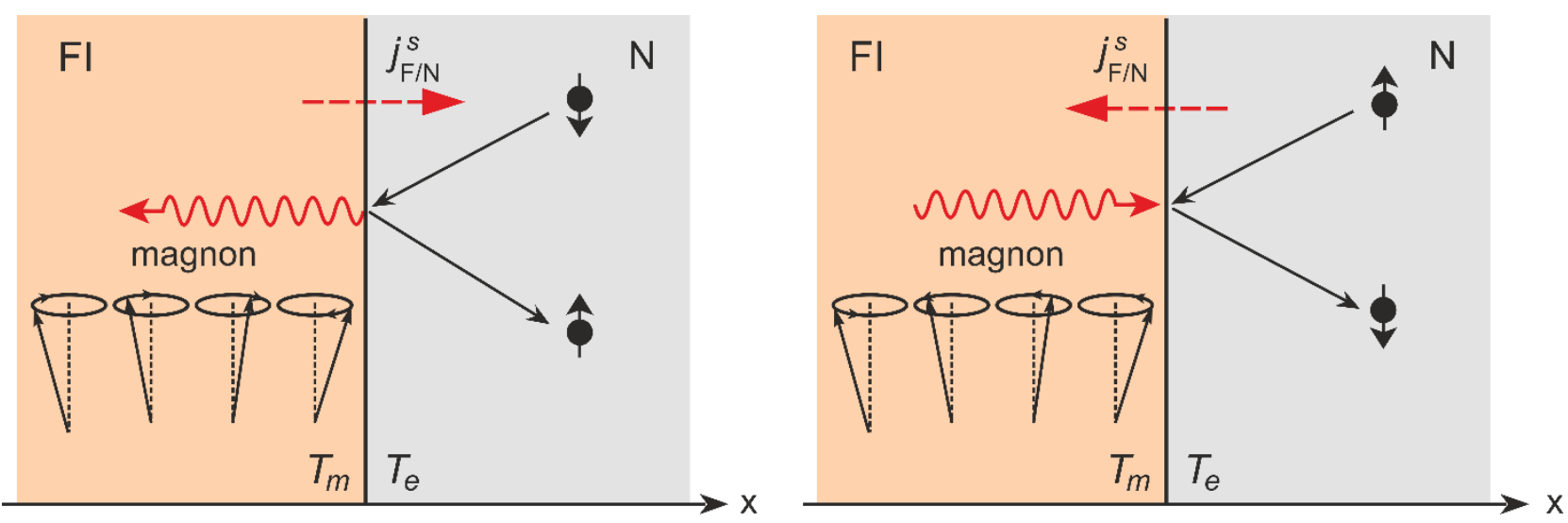

Figure 4.8: Illustration of quantum mechanical spin-exchange processes at a FI/NM interface. The two graphics $(a)$ and (b) show the magnon emission and absorption associated with the spinflip scattering of conduction electrons at the interface respectively, thus transferring spin angular momentum from FI to $N$ and from $N$ to FI through exchange interaction. [72] 


\section{CHAPTER 5 : SPECTROSCOPY ON MnF2}

This chapter presents the results of AFMR spectroscopy and the associated fitting simulations performed to characterize the $\mathrm{MnF}_{2}$ samples used in the studies presented in this thesis.

\subsection{Introduction}

Spectroscopy reveals valuable information on antiferromagnetic resonance such as resonance position, line width, etc. which provide great insight into the physics of a given antiferromagnetic system. In most EPR experiments, absorbance of microwaves is the standard way to find resonance position and to overcome the problem of weak signals, resonant cavities can be used. In a resonant cavity, standing waves are set up with their electric field and magnetic field exactly out of phase as shown in Figure 5.1.

At resonance, the resonant cavity stores microwave energy such that no radiation is reflected back. This is critical coupling of resonant cavity and is measured by the quality factor $Q$ of the cavity given as:

$$
Q=\frac{f_{o}}{\Delta f}
$$

where $f_{o}$ is the resonance frequency and $\Delta f$ is the width at half height of resonance. 


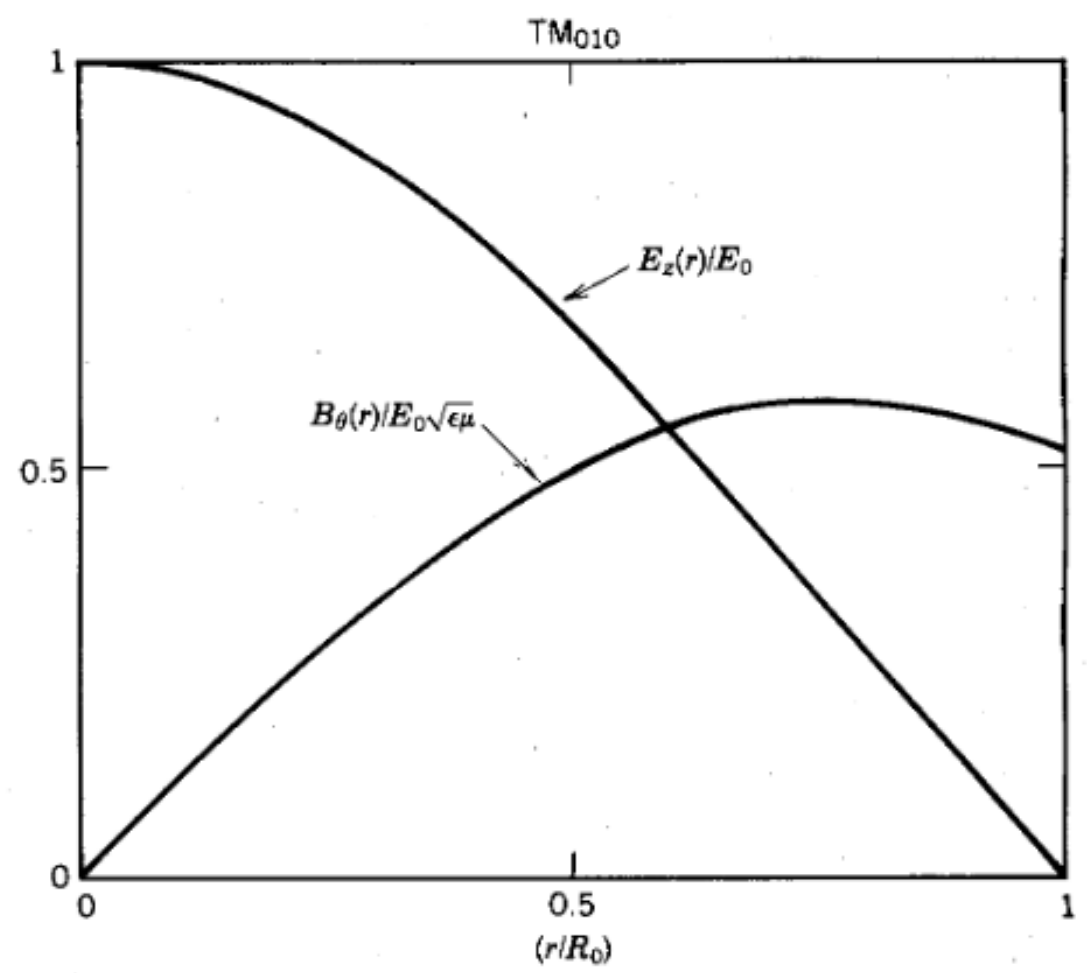

Figure 5.1: Normalized axial electric field and azimuthal magnetic field as a function of radius for TM010 mode in a cylindrical cavity

For measurement purposes, the sample is placed where the electric field is minimum and magnetic field is maximum. The sample absorbs microwave energy at resonance, lowering $Q$ and the cavity is no longer critically coupled. As a result, microwaves are reflected back and can be measured. Thus, the measure of absorption gives the resonance position for a sample. In EPR spectroscopy, microwaves of a certain frequency are sent to the cavity holding the sample using waveguides and magnetic field sweeps are taken. 
Over coupled

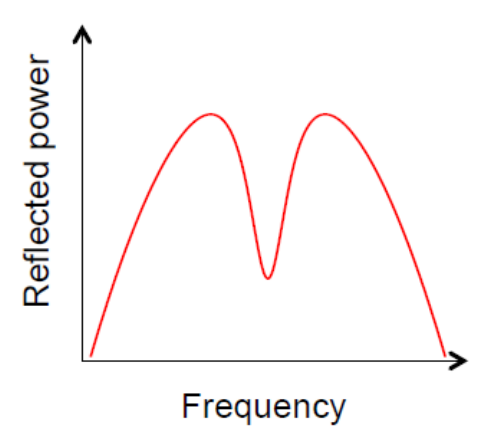

Critically coupled

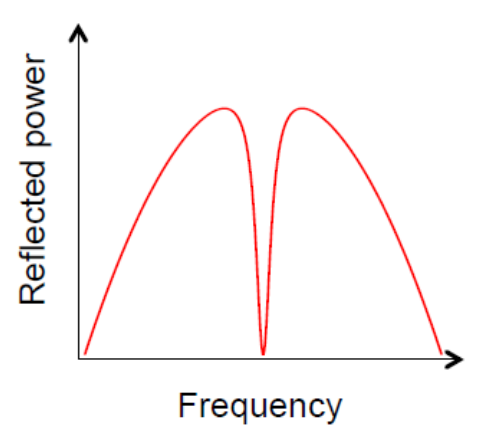

Under coupled

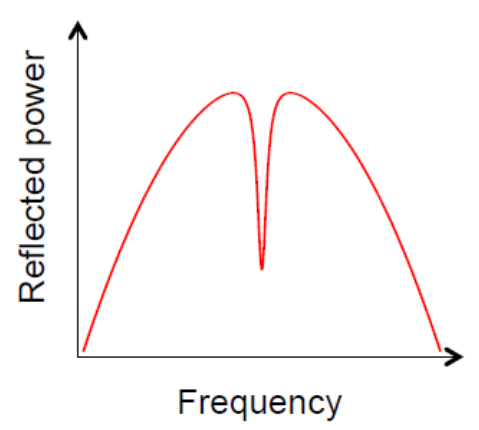

Figure 5.2: Illustration of differences in resonance signal between over-coupled, critically coupled and under coupled resonant cavity

EPR spectrometers use phase sensitive detection that enhances signal sensitivity significantly and diminishes noise. The noise in signal can be minimized by sinusoidal modulation of magnetic field at the sample site which ensure that if there is an EPR signal, field modulation quickly sweeps through part of the signal and reflected microwaves become amplitude modulated at the same frequency which can then be detected. Noises and electrical interference are not amplitude modulated and thus are not detected.

\subsection{Experimental Setup: Quasi-heterodyne Spectrometer}

All the experiments were performed at the National High Magnetic Field Laboratory, Tallahassee, Florida in the quasi-optical spectrometer with a heterodyne detection scheme. This spectrometer is designed for real time resolution and multifrequency operation and utilizes high frequency 
Schottky diode mixers with a detection bandwidth of the order of $1 \mathrm{GHz}$ [81]. Its quasi-optical design optimizes sensitivity and minimizes losses. It employs an induction-mode detection technique that includes circular corrugated waveguides. The direction of propagation is maintained parallel to the magnetic field [81]. The description for the setup is taken from [81].

This spectrometer does require dedicated mixers and detectors as well as a second high-frequency source to work as the local oscillator which limits the number of operative frequencies. Currently 4 frequencies are operable: $120 \mathrm{GHz}, 240 \mathrm{GHz}, 336 \mathrm{GHz}$ and $395 \mathrm{GHz}$ although interchanging the source and local oscillator allows a few more frequencies.

The basic schematic of the setup is shown in Figure 5.3. Phase locked Gunn diode $\left(\mathrm{G}_{2}\right)$ generates source millimeter waves of required frequency $\left(\mathrm{TE}_{01}\right.$ mode) which is then transformed to $\mathrm{HE}_{11}$ mode in a circular waveguide by a corrugated horn $(0.3 \mathrm{~dB}$ loss $)$ which allows this broadly Gaussian beam to gently expand to an overmoded aperture with zero phase curvature. From the horn, the beam enters the quasi-optical millimeter wave bridge (dotted box) where it first passes through a variable attenuator $(0-30 \mathrm{~dB})$ and then through a circulator located between a pair of wire-grid polarizers and consists of a $45^{\circ}$ Faraday rotator made up of a ferrite plate with antireflection coatings. The circulator acts as an isolator to dampen the standing waves and redirect the beam towards the reference detector. Beyond the circulator, the radiation enters the MartinPuplett interferometer that serves to convert the polarization of radiation by splitting the incoming linearly polarized beam into two $\pm 45^{\circ}$ polarized beams and then recombining them with a variable 
phase shift thereby converting a linearly polarized incoming beam into left or right handed circular polarization or into perpendicular linear polarization. The Martin-Puplett interferometer is particularly suited for our project as it is very useful in measurements involving thin films with the dc magnetic field in the plane of the film and the millimeter wave propagation direction perpendicular to the plane of the film and the dc magnetic field. The radiation emerging out of the interferometer is carried by corrugated waveguides (internal diameter: $18 \mathrm{~mm}$ for $120 \mathrm{GHz}$ and $22 \mathrm{~mm}$ for $240 \mathrm{GHz}$ ) to the sample.

The radiation that returns from the sample is split into two components by a wire grid polarizer. The co-polar beam is carried to the circulator and then onto the reference Schottky diode mixer $\left(M_{1}\right)$ while the cross-polar beam travels to the signal Schottky diode mixer $\left(M_{2}\right)$. To both these beams, another beam serving as a local oscillator signal is added which is generated by the second Gunn oscillator $\left(\mathrm{G}_{1}\right)$ which operates at a frequency which is $6 \mathrm{GHz}$ below the source frequency thereby generating IF signals of $6 \mathrm{GHz}$. The IF signals get amplified by low noise amplifiers, pass through variable attenuators and then $6 \mathrm{GHz}$ mixers $\mathrm{M}_{3}$ and $\mathrm{M}_{4}$ mix it down to de in a quadrature detection scheme. Phase tuning is achieved by a $6 \mathrm{GHz}$ phase shifter in the reference channel. The quadrature detection scheme is particularly useful in cases where a cavity or resonator are not applicable as a mixture of absorption and dispersion signals (mutually $90^{\circ}$ out of phase that allows decreased error) is measured which allows phase corrective manipulation to occur after data collection. The signals from the mixers $\mathrm{M}_{3}$ and $\mathrm{M}_{4}$ are then led to a pair of lock-in amplifiers (SR830) for field-modulated cw EPR or to a digital oscilloscope for transient EPR. 


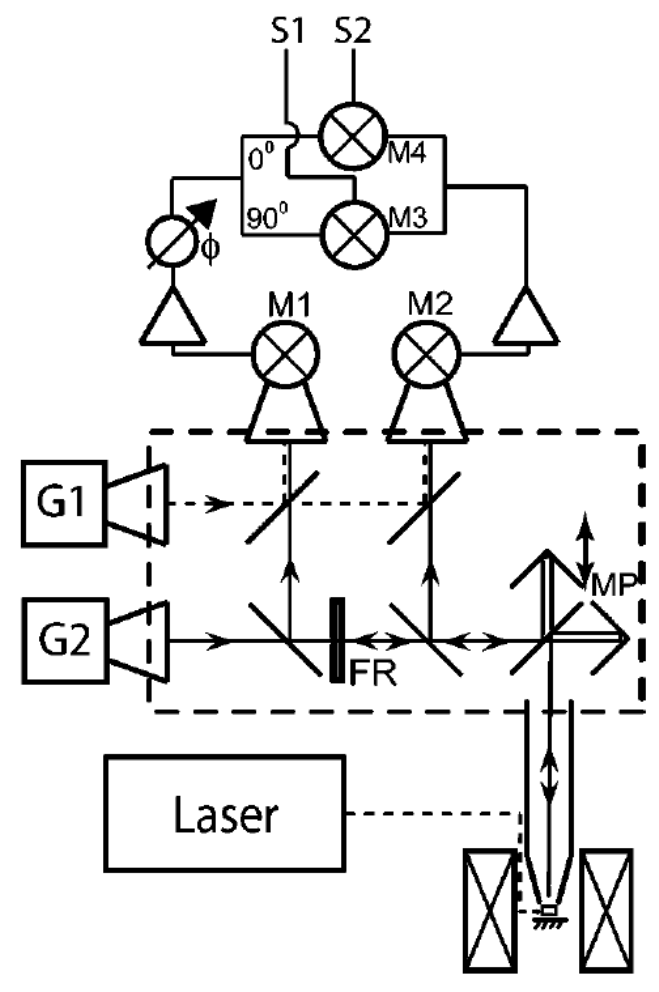

Figure 5.3: Experimental setup of the Quasi-Heterodyne Spectrometer at the National High Magnetic Field Laboratory, Tallahassee, FL[81]

\subsection{Sample Preparation}

For the work presented in this thesis, $\mathrm{MnF}_{2}$ was obtained from a large single crystal of $\mathrm{MnF}_{2}$ provided by Art Ramirez (Materials Advancement Portal at UC Santa Cruz). The crystal was cut by D. Lederman and S. Morley of Department of Physics, UC Santa Cruz, along the [001] direction and the resulting (110) surface was mechanically polished and acid etched to produce a smooth surface with RMS roughness of $0.9 \mathrm{~nm}$. 


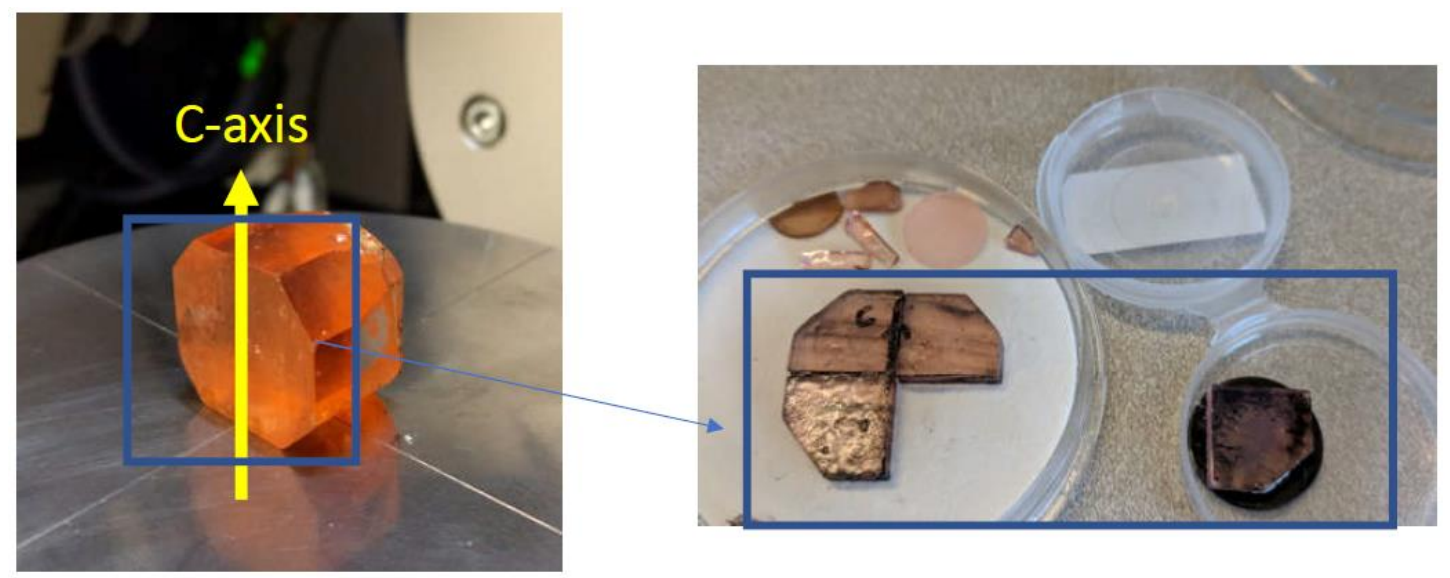

Figure 5.4: Precut single crystal of $\mathrm{MnF}_{2}$ that was divided into four quadrants. Three were used for sample preparation and the fourth was kept for characterizations. The size of each quadrant is about $10 \mathrm{~mm}$ by $10 \mathrm{~mm}$.

On this surface, $10 \mathrm{~nm}$ of $\mathrm{MnF}_{2}$ was grown as a buffer layer at $250^{\circ} \mathrm{C}$ via molecular beam epitaxy (MBE) at a base pressure of $1.1 \times 10^{-9}$ Torr and a growth rate of $0.003 \mathrm{~nm} / \mathrm{s}$ and then cooled close to room temperature of $28^{\circ} \mathrm{C}$. Finally, without breaking vacuum, $4 \mathrm{~nm}$ of Pt layer was deposited via electron beam evaporation to obtain a contaminant-free interfacial layer. The final RMS roughness of the top Pt layer was 1.6nm. 


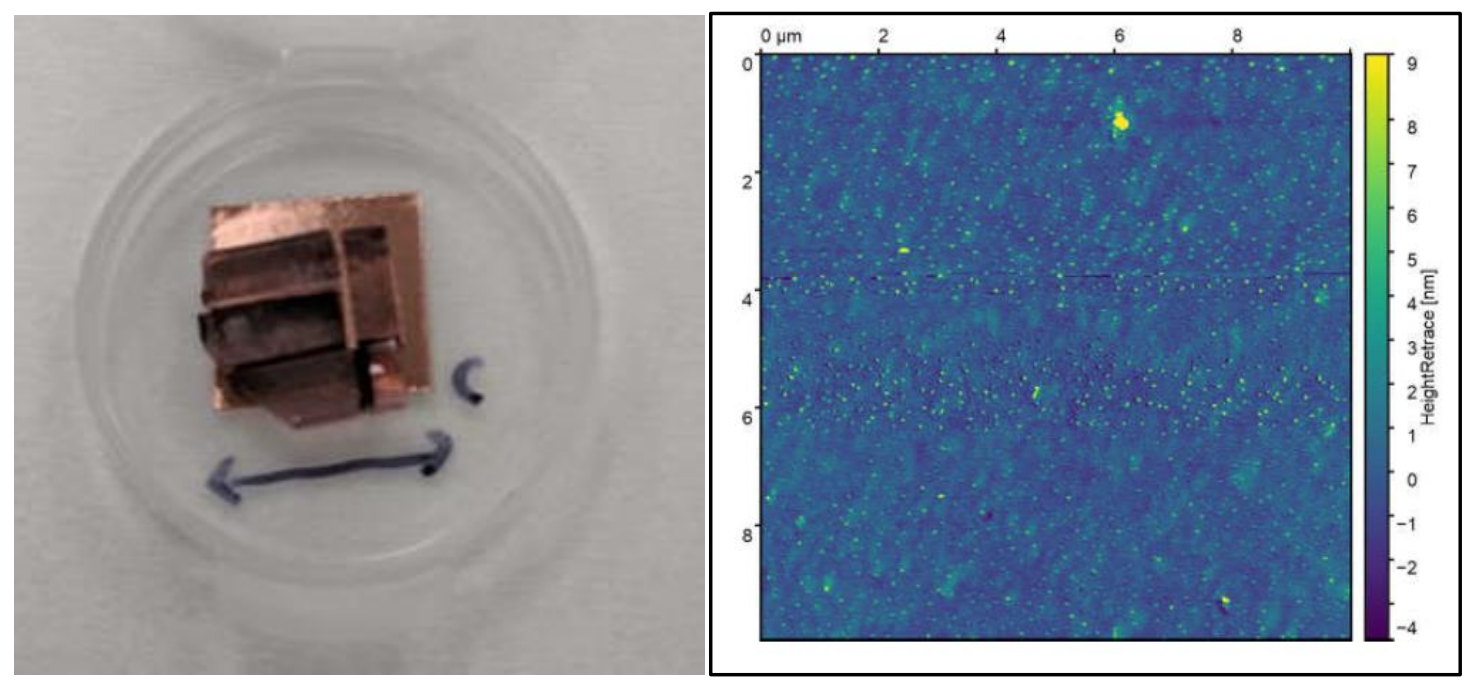

Figure 5.5: $\mathrm{MnF}_{2}$ single crystal/ $\mathrm{MnF}_{2} 10 \mathrm{~nm}$ thin film/ Pt 4nm heterostructure. On the left is the AFM image of the top layer of Pt which shows average RMS roughness of about 1.6nm.

\subsection{Sample Mounting}

The sample is prepared by cleaning the Pt surface with Acetone/Isopropanol. Then for spectroscopic measurements, the sample is mounted on a single axis rotator below the end of a quasi-optical feedhorn with an exit diameter of $5 \mathrm{~mm}$. The rotator allows for a continuous variation of the angle between the $\mathrm{MnF}_{2} / \mathrm{Pt}$ interface plane and externally applied magnetic fields with a precision of around 0.5 degrees. The direction of mounting is illustrated in Figure 5.5b. The sample has been cut such that the $\mathrm{c}$-axis of $\mathrm{MnF}_{2}$ is in plane of the sample and thus the mounting of sample is performed such that the field is applied along the plane of the sample. The sample rotator is then rotated to decrease the misalignment between the c-axis and the applied field with spectroscopic signal to guide in the process. 

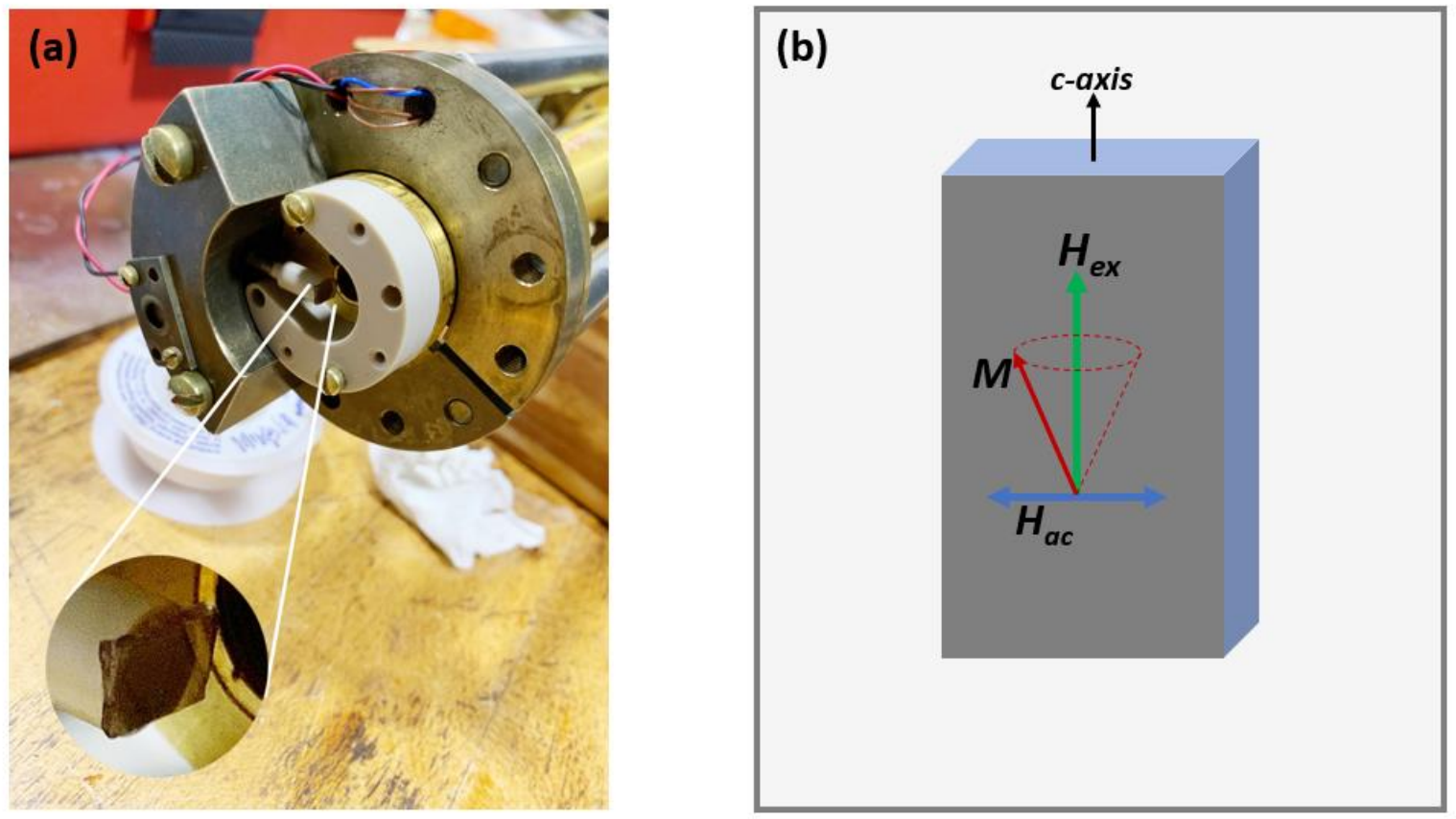

Figure 5.6: (a) Sample mounted for EPR measurements. (b) Illustration of sample mounting with respect to the external field, microwave field and the easy axis of the sample.

\subsection{Spectroscopic results on $\mathrm{MnF}_{2}$}

A total of three different samples were used to acquire the spectroscopic results presented in this section. Four different frequencies were sampled: $120 \mathrm{GHz}, 240 \mathrm{GHz}, 336 \mathrm{GHz}$ and $395 \mathrm{GHz}$, out of which, the former two belong to the low frequency mode and the latter two belong to the high frequency mode. Table 5.1 lists the samples and the frequencies they were sampled with. 
Table 5.1: List of samples used for the data presented in this thesis and the frequencies they were investigated at.

\begin{tabular}{||l|l|l|l|l||}
\hline & $120 \mathrm{GHz}$ & $240 \mathrm{GHz}$ & $336 \mathrm{GHz}$ & $395 \mathrm{GHz}$ \\
\hline Sample I & & & & \\
\hline Sample II & & & & \\
\hline Sample III & & & & \\
\hline
\end{tabular}

All AFMR measurements were performed with linearly polarized microwaves which can be visualized as superposition of two circularly polarized waves. All the expected frequency modes: low frequency AF resonant mode, high frequency AF resonant mode, spin-flop transition and high field spin-flop mode are observed across the three samples. For all the samples, signal is observed close to the expected resonance positions [21] for the individual frequencies. Several thicknesses of the sample, both on single crystal $\mathrm{MnF}_{2}$ and crystalline $\mathrm{MgF}_{2}$ were tested. While thin films on $\mathrm{MgF}_{2}$ substrate gave very broad signals, with thicker crystals of $\mathrm{MnF}_{2}$ as substrate the signals were very strong. This can be attributed to the fact that the size of $\mathrm{MnF}_{2}$ crystal is quite large with respect to the sensing area of the spectrometer resulting in a saturation of the sensitivity of the EPR probe. Considering the fragility of the sample, $200-300 \mu \mathrm{m}$ thick single crystal of $\mathrm{MnF}_{2}$ was chosen as substrate for the results presented here. The appendix I catalogues the signals obtained for both thin films on $\mathrm{MgF}_{2}$ substrate as well as thin films on $\mathrm{MnF}_{2}$ crystals. The signal is strong and rapidly oscillatory when $\mathrm{MnF}_{2}$ resonates with the microwave field corresponding to the different resonant modes as can be observed in Figures 5.8-5.10. Such signal is difficult to resolve to extract line width information and yet allow to determine the energy landscape associated with the AF modes 
of the sample and the corresponding misalignment of the easy axis with respect to the applied field. The position of resonance can be estimated with some degree of precision. This, coupled with the position of spin pumping data helps to find the exact position of the AF resonances as evidenced from Figure 5.7.

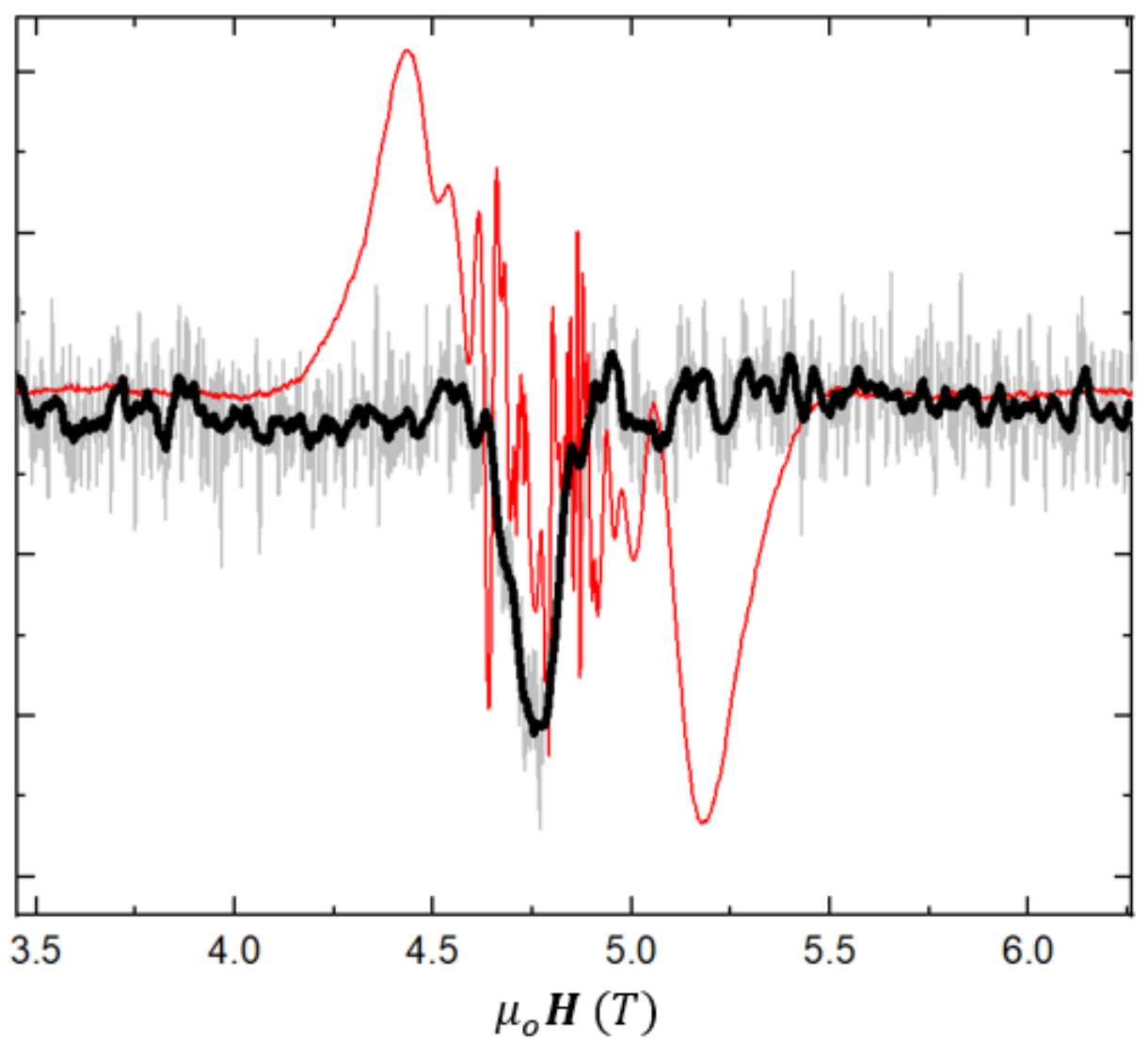

Figure 5.7: Spin-pumping data and AFMR data positions for Sample 3 for 395GHz at low-field high frequency resonance. The red line is the AFMR spectra while the black line is the averaged spin-pumping data. The grey line is the raw spin-pumping signal. 
The observed deviation from the exact resonance position is attributed to misalignment of the applied field from the easy axis of magnetization $\left(\theta_{H}\right)$. The value of $\theta_{H}$ not only determines the deviation of the signal but it also determines if the spin-flop signal is observed at low frequencies, as evidenced by Figure 5.10. The $120 \mathrm{GHz}$ signal for Sample 3 at the spin-flop transition is observed alongside the high frequency spin-flop mode signal due to a smaller degree of misalignment $\left(\theta_{H}<1^{\circ}\right)$. The spin flop transition was not observable in Sample 2, as a result of a larger degree of misalignment. The effect of misalignment is clarified further by the fitting results in the following section.

In Sample 1 and 2, all three resonance positions: low field, spin-flop transition and the spin-flop mode can be observed. This observation can be attributed to the misalignment in the sample. If there were no misalignment, the spin-flop mode resonance would occur at a field outside the range of the experimental setup preventing it from being observed. But by introducing misalignment, these modes can be investigated. In Sample 1, the misalignment is less than $1^{\circ}$ and so the signal at spin-flop mode for $240 \mathrm{GHz}$ appears at the very limit of the field as the maximum available field of 12.5T is insufficient to resolve this signal (see Figure 5.8). In Sample 2, the misalignment is larger, allowing for this signal to be more prominently visible (see Figure 5.9). 


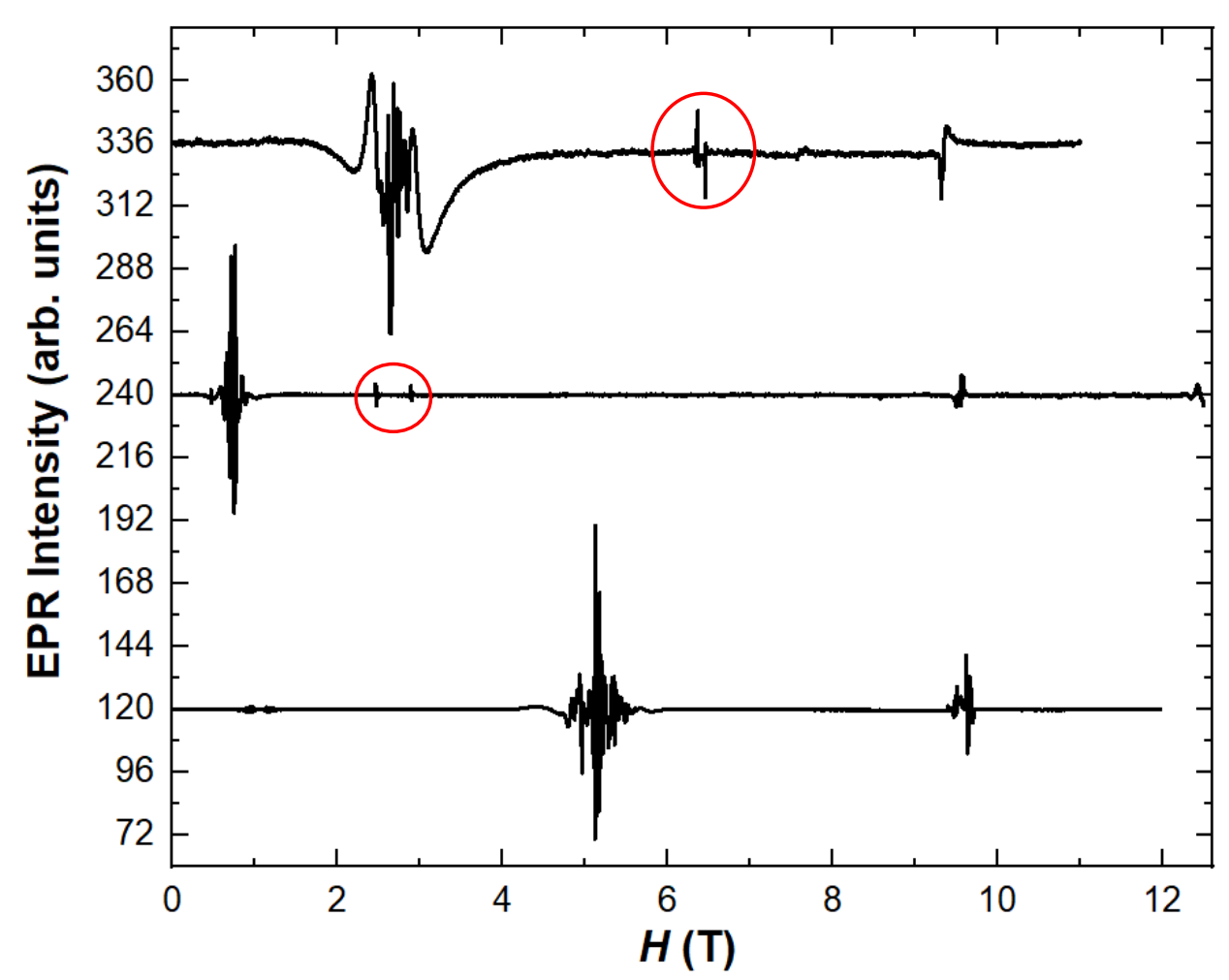

Figure 5.8: Spectroscopy results on Sample 1 for frequencies $120 \mathrm{GHz}, 240 \mathrm{GHz}$ and 336GHz. The red circles denote non-reproducible experimental artefacts and do not contribute to the actual data.

Some of the signals also show the presence of artifacts such as the ones for Sample 1 at $336 \mathrm{GHz}$ and 240GHz (Figure 5.8) as well as for Sample 2 at 240GHz (Figure 5.9). These artifacts can be attributed to features inherent to the measurement setup as they are not reproducible in different field sweeps. 


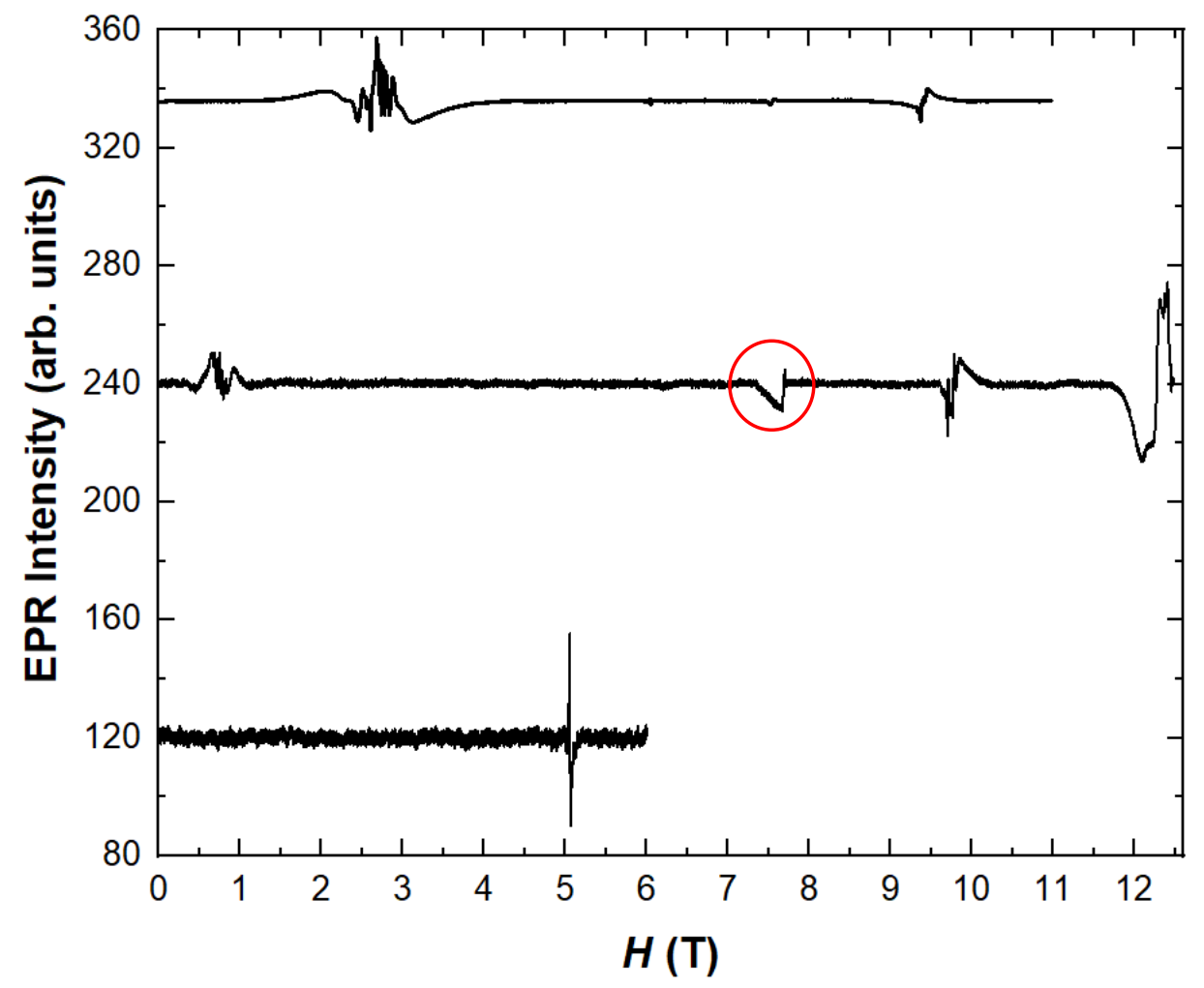

Figure 5.9: Spectroscopic results on Sample 2 for frequencies $120 \mathrm{GHz}, 240 \mathrm{GHz}$ and $336 \mathrm{GHz}$. The red circle denote non-reproducible experimental artifacts and do not contribute to the actual data. 


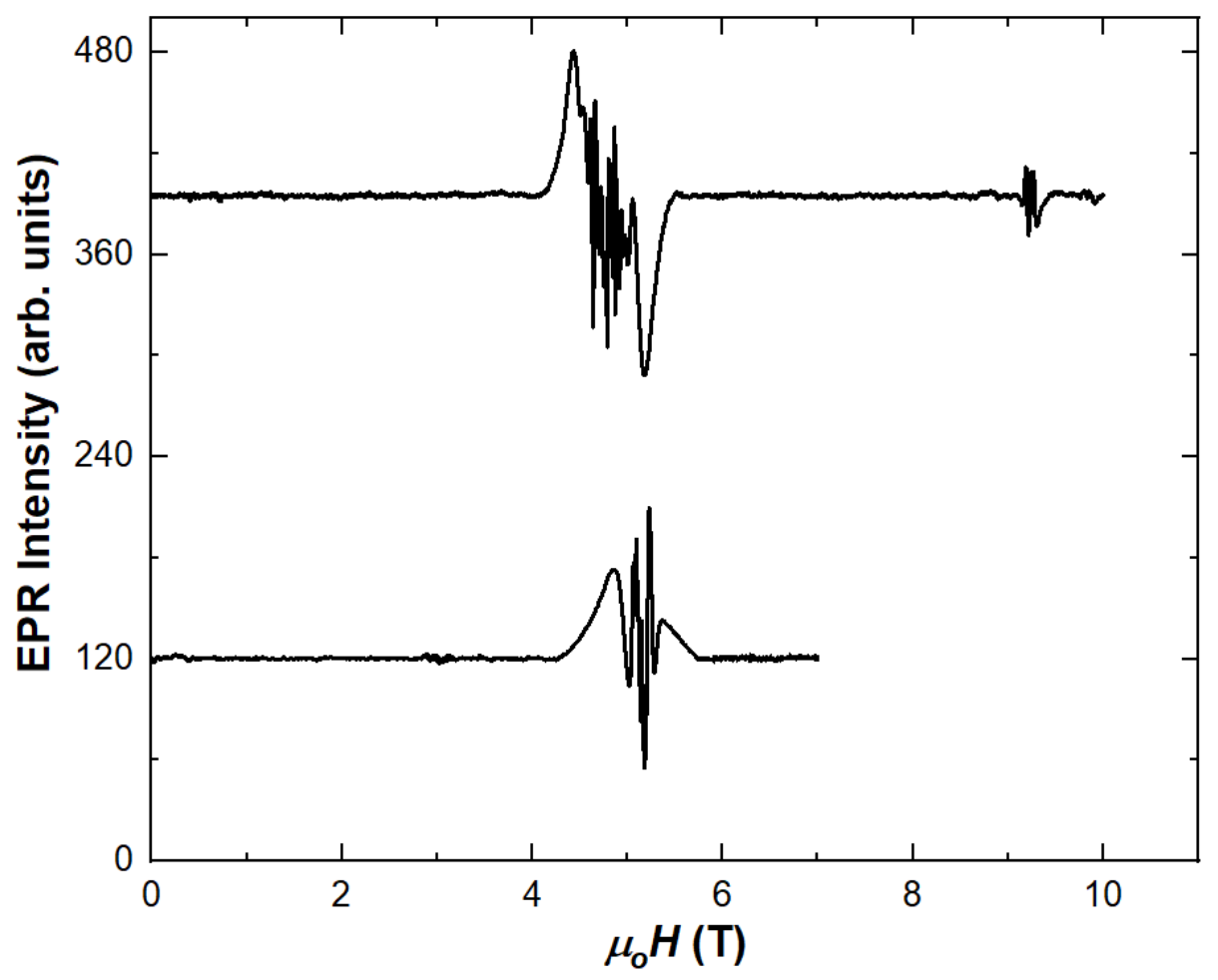

Figure 5.10: Spectroscopic results on Sample 3 for $120 \mathrm{GHz}$ and $395 \mathrm{GHz}$

\subsection{AFMR Fitting Results}

The AFMR results were fitted in a free energy approach using the mathematical formulation described in Section 3.4. These calculations are based on the formalism developed by Vonsovskii [55] and coded by Marc Philip Ross [44]. The codes were run in MATLAB® and the fitting parameters are listed in Table 5.2. Calculations were made for the magnetic field range used in the experiment. 
Table 5.2: Fitting parameters used in this thesis.

\begin{tabular}{|l|l|l|c|l|}
\hline Saturation & Exchange & Anisotropy Field & Gyromagnetic & Damping \\
Magnetization $(M s)$ & Field $\left(H_{E}\right)$ & $\left(H_{A}\right)$ & Ratio $(\gamma)$ & Constant $(\alpha)$ \\
\hline $0.06 \mathrm{~T}$ & $47.05 \mathrm{~T}$ & $0.8 \mathrm{~T}$ & $\gamma_{e}$ & 0.001 \\
\hline
\end{tabular}

The results of the fitting simulation are shown in Figure 5.11 for four different angles of misalignment. The parameters in the Table 5.2 were adjusted for best fit of the data in Sample 13. It can be observed that while for low-fields, the effect of misalignment is minimal, it becomes more pronounced at higher fields particularly around the spin-flop transition.

At smaller angles of misalignment, the three major regimes of AFMR spectra can be seen. For $H<H_{S F}$, there are two frequency branches as predicted by Kittel [56]. Misalignment has very little effect in this regime, particularly for $H \ll H_{S F}$. The zero-field degeneracy is calculated at about $263 \mathrm{GHz}$ for the fitting parameters listed in Table 5.2. For spin-flop transition $\left(H=H_{S F}\right)$, at zero misalignment, the high frequency branch suddenly drops towards zero frequency while the low frequency branch linearly reaches zero frequency. For increasing misalignment, there is a broader drop in the high frequency branch and has a smaller frequency span than the one for zero misalignment. The low frequency branch however does not completely reach zero frequency at $H=H_{S F}$. 


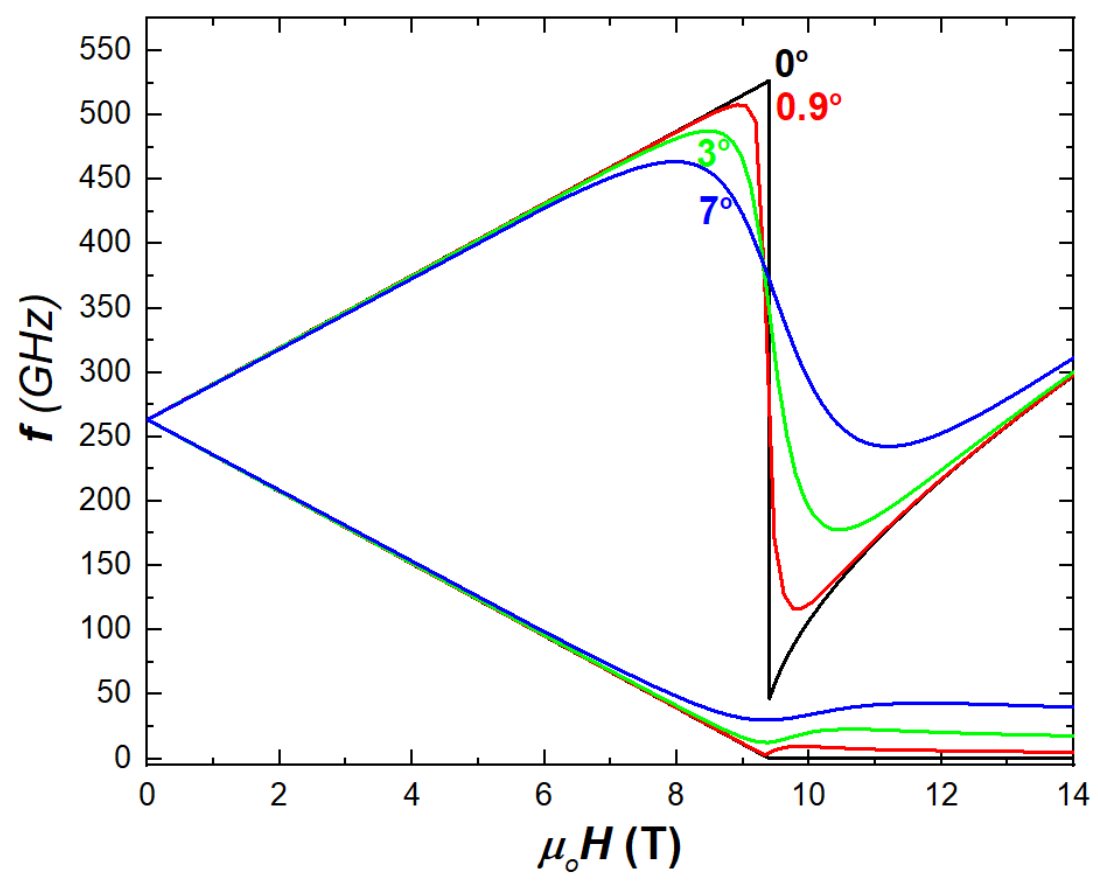

Figure 5.11: Calculated AFMR spectra for $M n F_{2}$ with four different misalignment angles that are color coded as follows: Black $=0^{\circ}$, Red $=0.9^{\circ}$, Green $=3^{\circ}$, Blue $=7^{\circ}$.

In the regime $H>H_{S F}$, for zero misalignment, only one non-zero frequency resonance mode exists in accordance with Nagamiya's results [57], which is the high frequency mode. This mode is called the spin-flop mode and has a quasiferromagnetic nature to it as explained in Section 3.3.1. The low frequency mode attains a zero value after $H=H_{S F}$. But with increasing misalignments, the low frequency mode attains a finite non-zero value that moves towards higher frequencies with increasing misalignments while the high frequency mode increases almost linearly with applied field for $H \gg H_{S F}$. 
The results of the simulation agree very well qualitatively with the established results of Hagiwara et.al. [21]. The fitting parameters differ from the ones used by Kittel which results in a different value for zero-field resonance as well as spin-flop transition but fits very well with the data as seen in the following section. Kittel used an effective field approach while this simulation is based on a free energy approach. Results obtained by Kittel and Nagamiya describe the regimes $H<H_{S F}$ and $H>H_{S F}$ respectively while this approach is able to calculate the AFMR spectra across the two regimes as well as the spin-flop transition which is yet to be understood fully. Thus, this simulation is utilized to fit the AFMR data.

The results of fitting data points with simulation are shown in Figure 5.12 and it can be observed that the simulation fits the AFMR data obtained in $\mathrm{MnF}_{2}$ very well.
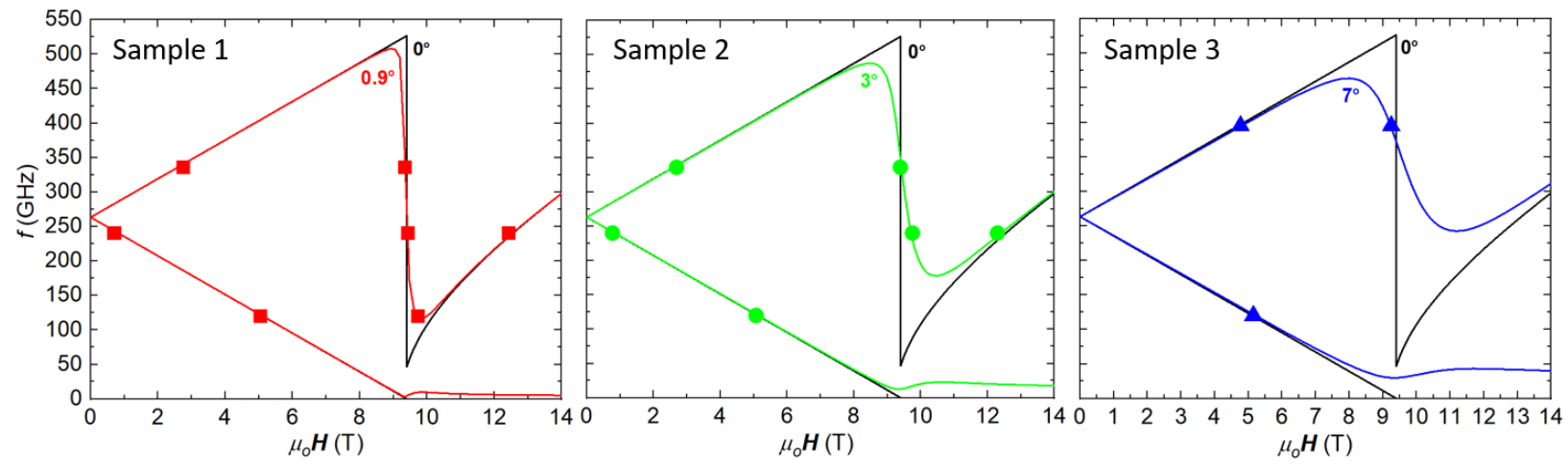

Figure 5.12: AFMR data fitted with simulation for the three samples. The symbols represent AFMR data points while the solid lines are obtained from simulation. Each sample has a different misalignment angle and the zero misalignment data is also represented for comparison in each chart. 
All three samples used came from a single original sample cleaved in several pieces, so no structural or magnetic changed are expected. The only difference relies on the misalignment of the different samples with respect to the direction of application of the magnetic field. Different samples were required for a complete data set as even a small misalignment introduces strong magnetic torque due to strong magnetic fields forcing the sample to detach from the sample holder with high velocity. Since the sample is rather fragile, such motion resulted in the sample to break into multiple pieces and ultimately be unrecoverable. With each new mounting, the rotator attached to the sample holder can be used to rotate the sample in plane and minimize the misalignment to a precision of $0.5^{\circ}$. However, such rotation can be safely performed only at low fields where the effect of misalignment is not so pronounced. While efficient minimization of misalignment is possible at higher fields, any movement away from zero misalignment induces torque on the sample making the process quite challenging. As a result, we have different misalignment angles for the three samples.

It can be observed through fitting in Samples 2 and 3 that for larger misalignments, the spin-flop transition and high field spin-flop mode become unobservable for low frequencies. This shows the sensitivity of AFMR spectra towards misalignment of applied field from the easy axis. Of the four sampled frequencies, $120 \mathrm{GHz}$ is most prone to misalignment as even a misalignment of $\theta_{H} \sim 1^{\circ}$ prevents the high field signals to be observed. However, for our spin-pumping measurements, $240 \mathrm{GHz}$ provides enough qualitative data that misalignment becomes less concerning. In fact, for the high-field spin-flop mode, the presence of misalignment allows the signal to be observed and studied for $240 \mathrm{GHz}$ within the available range of magnetic fields (maximum of $12.5 \mathrm{~T}$ ), since the 
misalignment shifts the SF mode to lower fields. This can be easily observed in Figure 5.8, where for a small misalignment angle $\left(\theta_{H} \sim 1^{\circ}\right)$ between the anisotropy axis and applied field, the maximum available field of $12.5 \mathrm{~T}$ is not sufficient to resolve the spin-flop mode at $240 \mathrm{GHz}$, while the spin-flop transition mode and high field spin-flop mode are so close that cannot be resolved. For larger frequencies of $336 \mathrm{GHz}$ and $395 \mathrm{GHz}$, the spin-flop mode is out of reach. To solve this issue, the samples were rotated to establish a misalignment between the easy axis and the applied field resulting in a broadening of the spin-flop transition and a low-field shift of spin-flop mode towards lower frequencies as seen in the case of Sample 2.

\subsection{Theoretical calculation of the net magnetization}

The projection of net magnetization of the system for a given applied field and a given misalignment was calculated from the equilibrium orientation of the magnetization vectors at that field. Figure 5.13 shows the various projections of net magnetization of the system $\left(\boldsymbol{M}_{n e t}=\boldsymbol{M}_{1}+\right.$ $\boldsymbol{M}_{2}$ ) at equilibrium as a function of the applied magnetic field for the three misalignment angles between the easy axis (z-axis) and the applied field that best fit the three samples. 


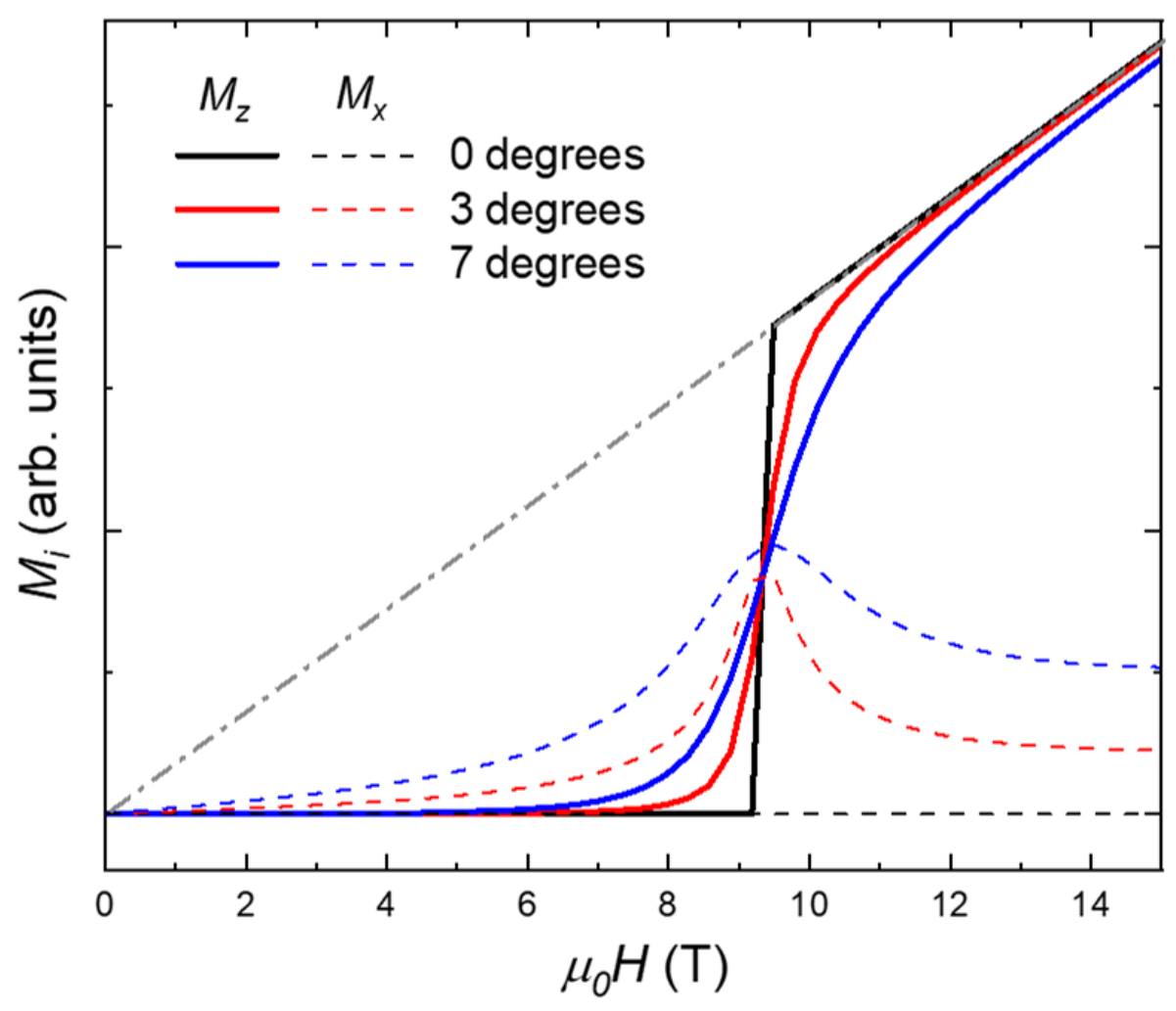

Figure 5.13: Net Equilibrium Magnetization in $\mathrm{MnF}_{2}$ : Projections of the net equilibrium magnetization $\left(M=M_{1}+M_{2}\right)$ in the $x$ and $z$ directions for the three misalignment angles $\left(\theta_{H}\right)$ between the anisotropy axis $\left(H_{A} \| z\right)$ and the applied field with the misalignment occurring in the $x$-direction. The grey dashed line represents $\theta_{H}=90^{\circ}$.

It can be observed that for $\theta_{H}=0^{\circ}$, there is no net magnetic moment below $H<H_{S F}$. It develops abruptly at $H=H_{S F}$ and then increases linearly with field for $H>H_{S F}$. For $\theta_{H}=3^{\circ}$ and $\theta_{H}=7^{\circ}$, the net magnetic moment increases gradually as field approaches $H_{S F}$. For sufficiently low applied fields $\left(H \ll H_{S F}\right)$, the lattice magnetizations completely cancel at equilibrium and the net spin only arises due to the differences in the precessional cones of the two magnetization vectors. Such 
precessional motion is different in different $\mathrm{AF}$ modes and thus the net magnetic moment is oriented in different directions for such modes: along the field (positive moment) for the low frequency mode and against the field (negative moment) for high frequency mode. 


\section{CHAPTER 6 : ISHE RESULTS ON MnF2}

This section presents the results and corresponding analysis of spin-pumping measurements on $\mathrm{MnF}_{2}$. The most relevant data are presented here for analysis, while some complementary data will be presented in Appendix II.

\subsection{ISHE Voltage Measurement Setup}

For spin-pumping measurements, the standard procedure to acquire ISHE voltage is adopted. Two leads are placed on the Pt surface of the sample along the edges parallel to the c-axis using commercially available silver paint. The leads are connected to micro-coaxial transmission lines that feed the signal to a lock-in amplifier (SR 850). A 50\% duty cycle pulse is applied, and voltage is measured after passing through a pre-amplifier. An illustration of the sample and contact directions can be found in Figure 6.1. The sample and the rotator are mounted at the end of a probe stick made of corrugated waveguides that is then put inside a cryostat with flowing liquid Helium. All measurements are done at $3.5 \mathrm{~K}$. 


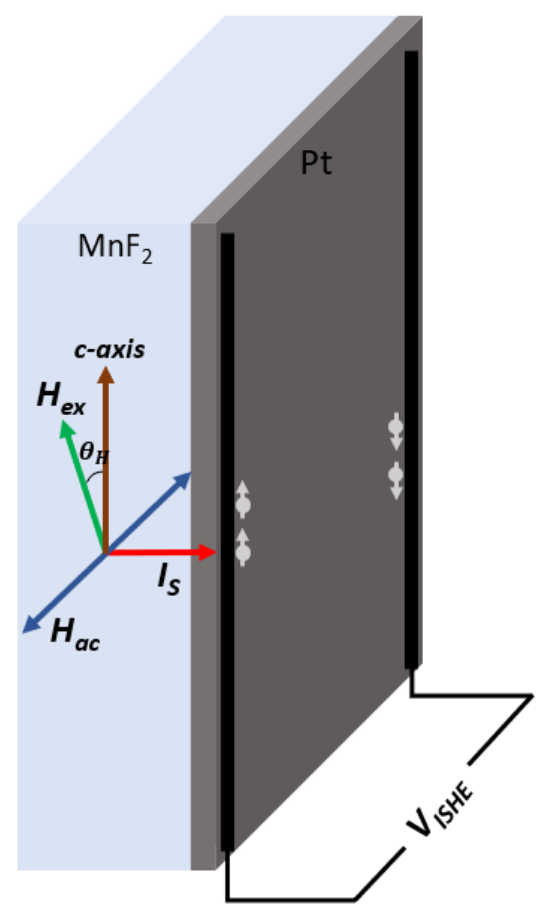

Figure 6.1: Illustration of the device geometry for spin pumping and ISHE measurements. The misalignment angle $\theta_{H}$ is to be minimized by rotating the sample.

\subsection{ISHE results on $\mathrm{MnF}_{2}$}

When spin pumping due to precessing magnetization occurs across the interface of a non-magnetic material and a magnetic material, a transverse voltage appears across the non-magnetic material via the ISHE, provided a sizable spin-orbit coupling (SOC) in the non-magnetic layer. Therefore, the ISHE voltage can then be used as a measure of spin pumping across the interface. 
In our experiments, polarized microwaves were applied and clear ISHE voltage signals associated with the antiferromagnetic resonances were observed for all three samples and at all four frequencies. A monotonous signal background has been subtracted from all the ISHE spectra and Figure 6.2 shows a typical ISHE signal obtained for $336 \mathrm{GHz}$ in Sample 2. Clear signals are observed for both the low field resonance of the high frequency mode and spin-flop transition consistent with the EPR spectra of $336 \mathrm{GHz}$. As can be observed, the signals reverse sign when the applied field is reversed which is consistent with time reversal symmetry. The handedness of the applied microwaves was also reversed, and the effect was observed in the magnitude of the ISHE signal. The strength of the signal at spin-flop transition varied with reversal of field and reversal of circular polarization. The effect on the low field resonance mode was even more stark as the signal appeared only at positive field (2.69T) for right-handed circular polarization and then for left-handed circular polarization, the signal appeared only at negative field (-2.69T) with opposite polarity. 


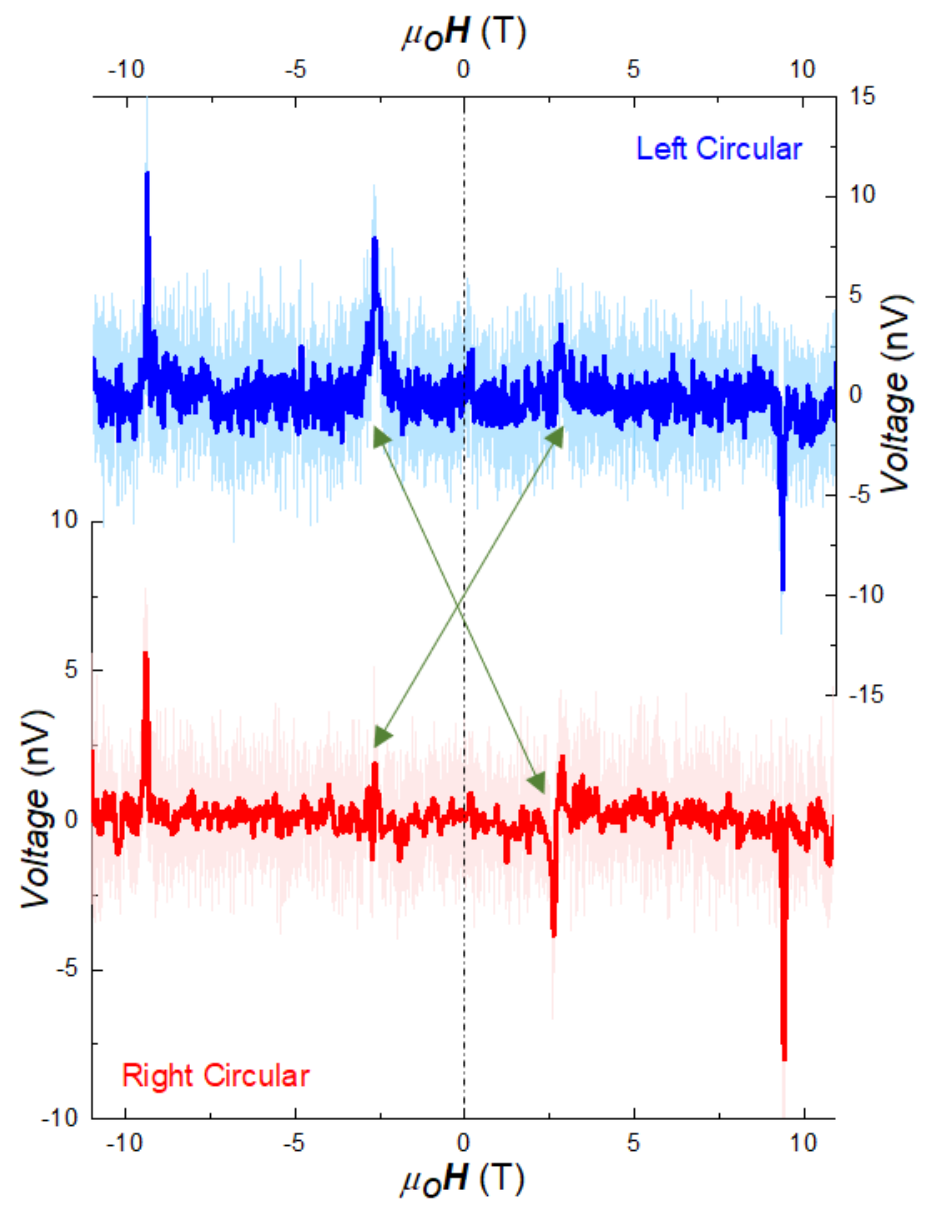

Figure 6.2: ISHE signal for Sample 2 at 336GHz. The voltage signal correspond to the resonance position observed in spectroscopic measurements.

Cheng et al. developed the theoretical framework to understand dynamical spin injection from an AF material undergoing coherent precession (AFMR) into an adjacent non-magnetic material [45] (see also [46]). Contrary to the conventional wisdom that spin pumping from antiparallel sublattice spins would cancel out, Cheng et al. established that coherent resonant rotations of different sublattice spins contribute constructively to the pumped spin current. Consequently, it is the Néel 
vector $\boldsymbol{L}$, rather than the vanishingly small magnetization $\boldsymbol{M}$, generates the most essential part of coherent spin pumping. Furthermore, it was predicted in [45] that the polarization of the driving ac field determines the direction of the pumped spin current because dynamical modes with opposite chirality coexist in a collinear AF system; and they can be selectively excited by an ac field with matching polarization. In other words, spins are pumped either parallel or antiparallel to the applied magnetic field depending on whether the right- or left-handed mode is excited (by a right- or left-handed circularly polarized stimulus). Therefore, by changing the handedness of the circular polarization and the frequency of irradiation at a given magnetic field, opposite spin currents are generated in the adjacent non-magnetic material and transform into opposite ISHE electric signals.

The measured ISHE spectra in samples 3 and 2 are shown in Figure $6.3(2 \mathrm{a}-\mathrm{b})$ for $f=395 \mathrm{GHz}$ and 6.3 (2c-d) for $f=240 \mathrm{GHz}$, respectively. Figure 6.4 shows the power dependence data for $f=395$ GHz. For $f=240 \mathrm{GHz}$, clear voltage signals were observed associated with the spectroscopic spectra for the LFM, the SF transition, and the SFM. All signals reversed sign when the applied magnetic field reversed direction, which is consistent with the time reversal symmetry. But their magnitudes were apparently different under the reversal of the handedness of the microwave stimuli, implying that chiral AF modes were selectively excited according to the circular polarization. This contrasting magnitude becomes the most striking feature in Figure 6.3 (c) and (d), where the LFM only appears at a positive (negative) field $\left(\mu_{0}|H|=0.80 \mathrm{~T}\right)$ for the left (right)handed irradiation. This is indeed the expected behavior of a circularly-polarized AF mode in the 
presence of an external magnetic field. For positive (negative) fields, the LFM mode's chirality is left (right)-handed as it has a spin angular momentum parallel to the magnetic field, while the opposite is true for the HFM. There is also a noticeable difference in the strength of the SF signals by reversing only the magnetic field or only the circular polarization. On the other hand, the magnitude of the SFM resonance basically remains constant, which we will discuss further below.
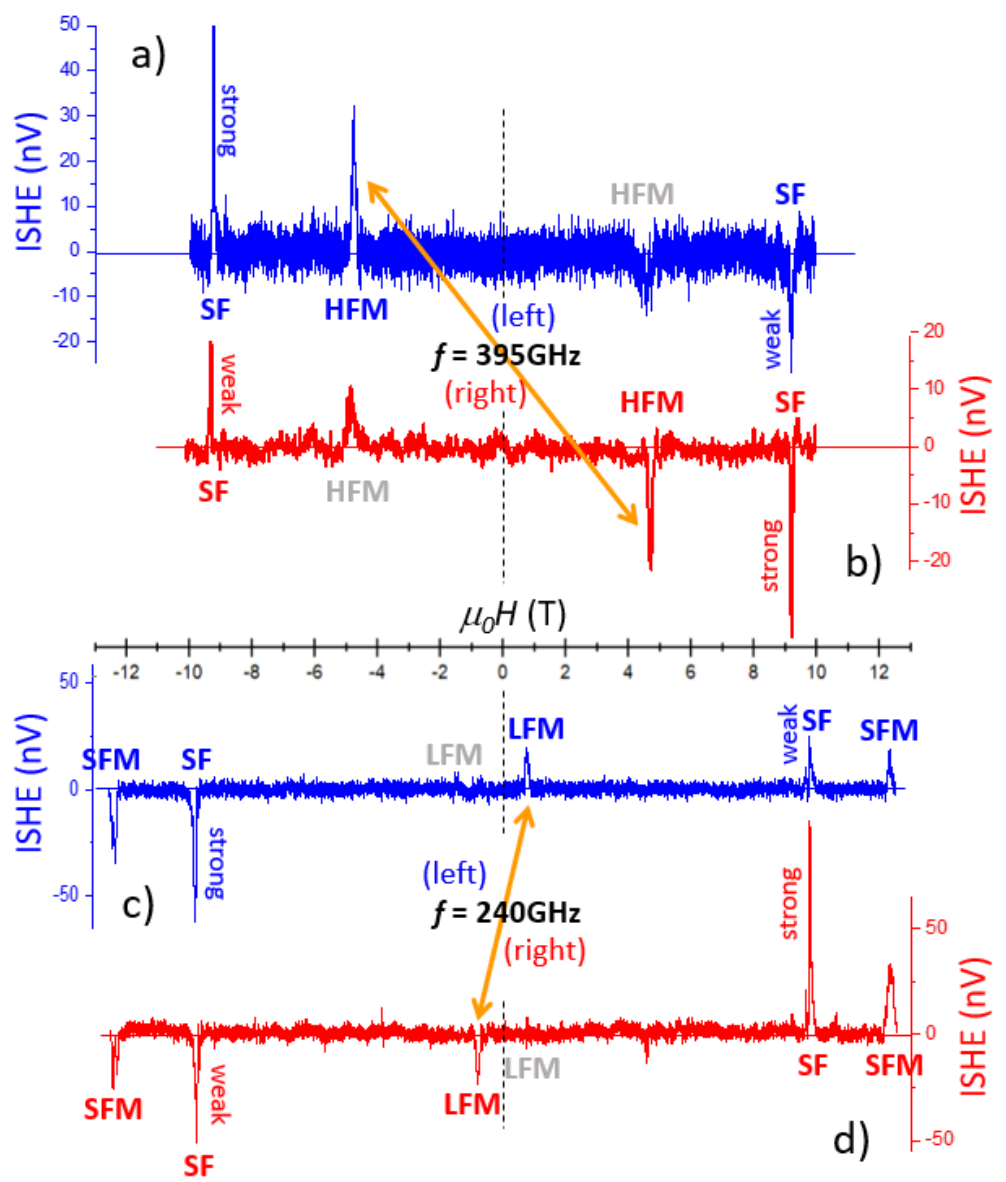

Figure 6.3: ISHE signal obtained in Sample 3 at 395GHz for (a) left-handed (b) right-handed circularly polarized microwaves and in Sample 2 at 240GHz for (c) left-handed (d) right-handed circularly polarized microwaves. 


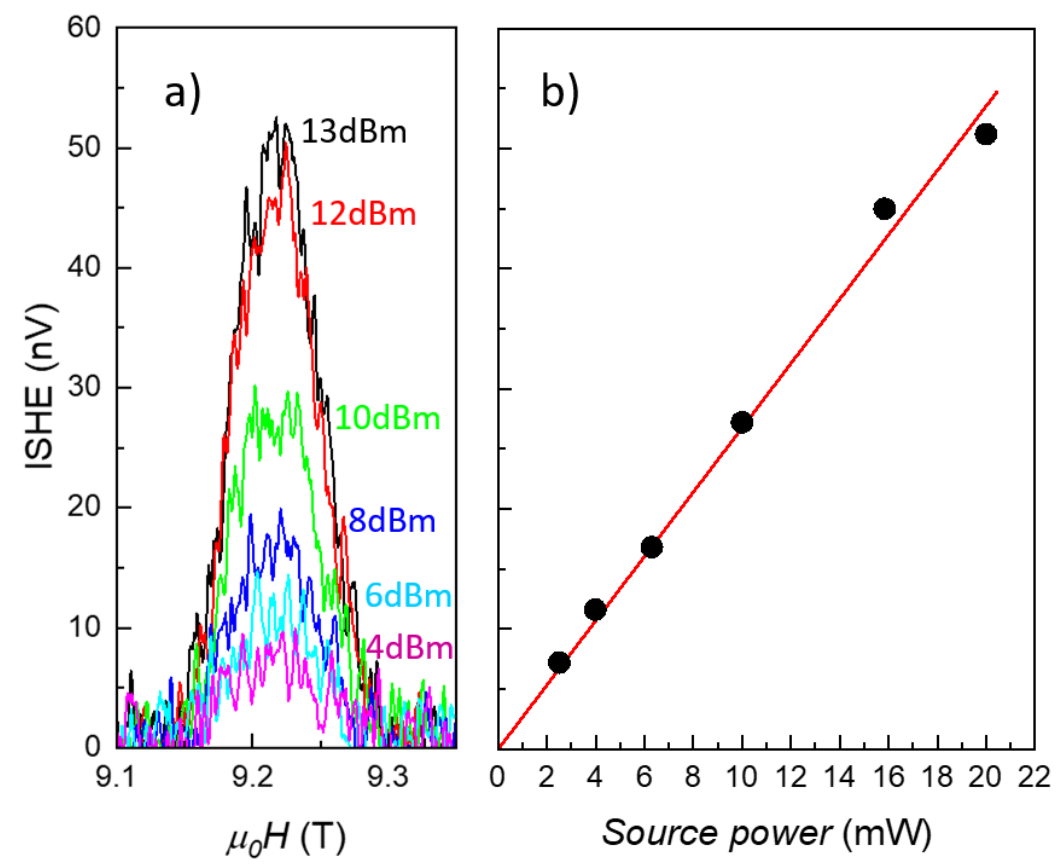

Figure 6.4: Power Dependence of the ISHE: (a) ISHE signal obtained at the Spin-Flop transition in Sample 3 at $395 \mathrm{GHz}$ for different powers of the microwave source and (b) corresponding power dependence with a linear fit.

\subsubsection{Coherent Spin Pumping vs Incoherent Spin-Seebeck Effect}

A central question arises from these observations: Do the voltage signals originate from coherent spin pumping at the $\mathrm{MnF}_{2} / \mathrm{Pt}$ interface, or the incoherent spin Seebeck effect [18] induced by a temperature gradient resulting from microwave heating? In ferromagnets, this is quite a challenging question since only the right-handed mode exists, thus both coherent and incoherent contributions have the same spin polarization that electrical measurements alone cannot 
distinguish [82]. The situation is fundamentally different in antiferromagnets. The coexistence of both chiral modes in AF systems allows us to discern between coherent and incoherent contributions from the electrical measurements alone. The high frequency $(395 \mathrm{GHz})$ data for Sample 3 in Figure 6.3 2a-b (see analogous data for Sample 2 in Appendix B and related discussion) indicate that electric signals from the HFM resonance (at $H= \pm 4.7 \mathrm{~T}$ ) behave in the exact opposite way than the LFM when switching polarities. The HFM signal only appears at positive (negative) fields with the right (left)-handed irradiation, as it corresponds to the right (left)-handed chirality of the excited AF mode. On the other hand, the sign of spin Seebeck effect would be independent of the microwave handedness, because it always primarily originates from the LFM mode (thermally more populated than the HFM) even if the microwave heating stems from the resonant absorption of microwave energy by the HFM. Therefore, our experimental observation unambiguously demonstrates that the effect originates from coherent spin pumping and the ISHE in Pt.

Given the unambiguous coherent origin of the signals, we can further estimate the spin-mixing conductance of the $\mathrm{MnF}_{2} / \mathrm{Pt}$ interface from the measured ISHE voltage. Taking into account the back-flow of spin current in the Pt layer, and scaling the spin-mixing conductance into the areal density of $e^{2} / h$, we obtain $[83,84]$ :

$$
V_{I S H E}=L \theta_{S}\left(\frac{H_{A}}{H_{E}}\right)\left(\frac{\lambda}{d_{N}}\right)\left(\frac{\hbar e\left(\gamma B_{\perp}\right)^{2}}{\alpha^{2} \omega_{R}}\right)\left(\frac{g_{r} \tanh \frac{d_{N}}{2 \lambda}}{h \sigma+2 \lambda e^{2} g_{r} \operatorname{coth} \frac{d_{N}}{2 \lambda}}\right)
$$


where $L$ is the distance between the two voltage leads; $d_{N}, \theta_{S}, \lambda, \sigma$ are the thickness, the spin Hall angle, the spin diffusion length and the conductivity of the Pt layer; $\alpha$ is the Gilbert damping in $\mathrm{MnF}_{2} ; \omega_{R}$ is the angular frequency of AFMR and $B_{\perp}$ is the amplitude of the magnetic field of the circularly-polarized microwaves. The following values have been used from literature: for $\mathrm{MnF}_{2}$, $\frac{H_{A}}{H_{E}} \approx 1.8 \%$ and $\alpha \approx 0.5 \times 10^{-3}$; for Pt layer, $d_{N} \approx 4 \mathrm{~nm}, \theta_{S} \approx 0.04, \lambda \approx 4 \mathrm{~nm}$ and $\sigma \approx$ $2 \times 10^{6} \mathrm{~S} / \mathrm{m}$. At the peak (dip) of $240 \mathrm{GHz}$ resonance, $B_{\perp} \approx 200 \mathrm{mG}$ and $V_{I S H E} \approx 25 \mathrm{nV}$. Using these values in Eqn. 6.1, the spin mixing conductance is estimated as $g_{r} \approx 4.3 \times 10^{18} \mathrm{~m}-2$, which

converts to $\approx 1 e^{2} / h$ per unit cell area on the interface. This value, though a rough estimate due to uncertainty in some of the parameters, is very consistent with the theoretical prediction $[45,46]$. Here we point out that the extracted spin-mixing conductance is of a similar magnitude compared to that in ferromagnet/normal metal heterostructures, which confirms the theoretical picture that opposite sublattice magnetizations can constructively pump spins, not cancel.

\subsubsection{Spin-flop transition and the high magnetic field spin-flop mode}

Now we consider the behavior of the SF and SFM signals. While the handedness of the microwave polarization modulates the SF resonance, it does not affect the SFM resonance. As can be seen in Figure 6.3, for positive (negative) fields, the strength of the SF resonance is more pronounced when the polarization is right (left)-handed, which is the case for both frequencies. In other words, the modulation of the SF resonance with circular polarization seems to follow the behavior of the HFM resonance. This feature is not surprising as the HFM retains its essential characteristics while 
the system undergoes a gradual evolution of spin configuration from the collinear AF ground state into a high-field spin-flop state. After the spin-flop transition, the spin dynamics starts to exhibit some characteristics of a ferromagnet as the total magnetization grows with an increasing magnetic field until the remaining HFM characteristics eventually disappear.

Figure 6.5 highlights this result more clearly, where we show all ISHE signals as functions of the relative phase that determines the circular polarization of the microwaves. While both the LFM and the HFM clearly show oscillatory patterns, the SFM signal has an essentially constant magnitude. On the other hand, the SF resonances display a mixture of both regimes - they oscillate on top of a constant background signal, which seems to coincide with the magnitude of the SFM at $240 \mathrm{GHz}$ (see the connecting arrow in Fig. 31). Figure 6.7 shows a direct comparison of the magnitudes of the LFM, SF and SFM signals observed at $f=240 \mathrm{GHz}$ for both positive and negative magnetic fields. 


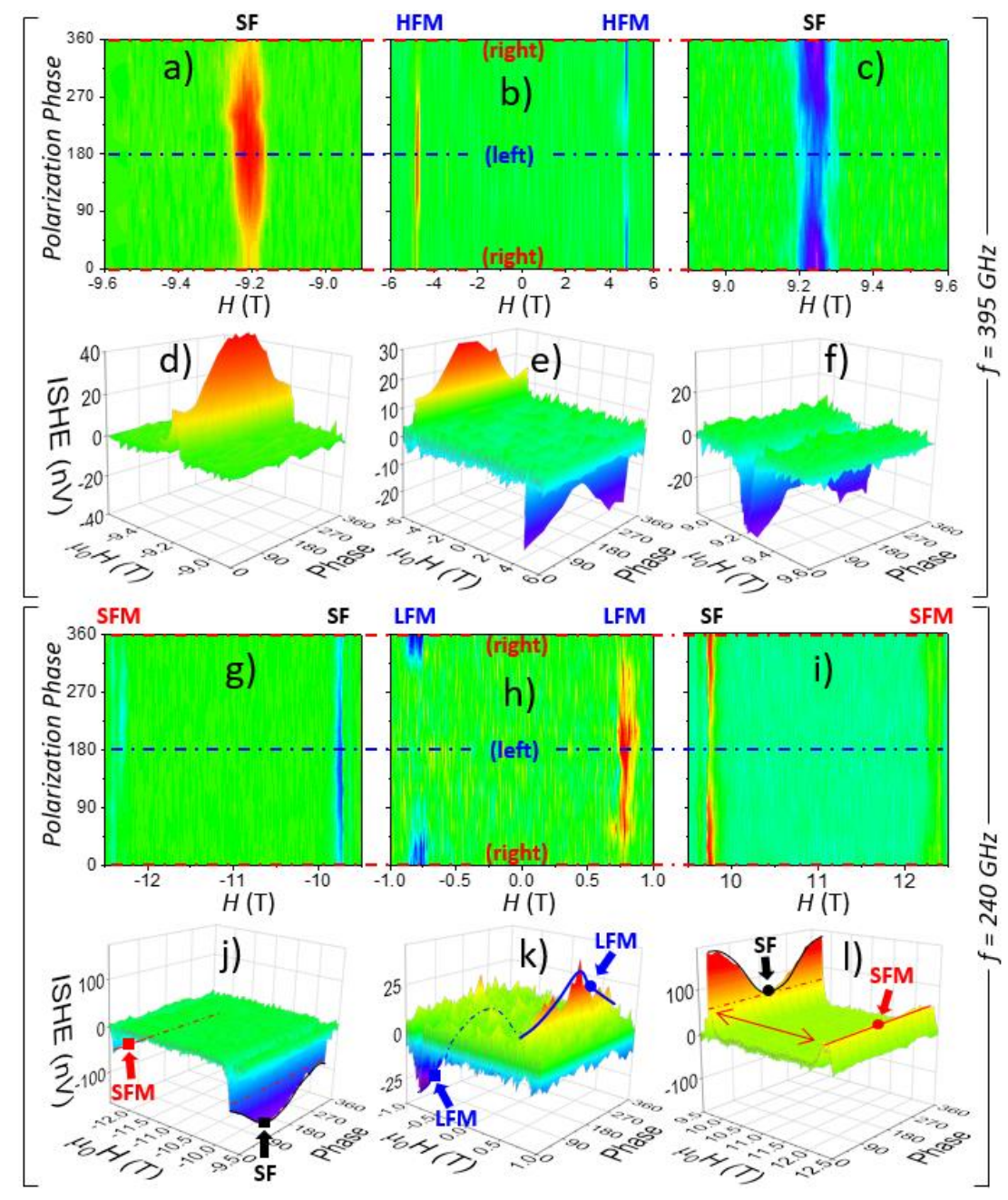

Figure 6.5: Circular Polarization Modulation of Spin Pumping: Evolution of the ISHE signals with applied field as a function of the polarization of the microwaves for (a-f) $395 \mathrm{GHz}$ and (g-l) $240 \mathrm{GHz}$. Polarization phases $0^{\circ}$ and $360^{\circ}$ correspond to right handed circular polarization and polarization phase $180^{\circ}$ corresponds to left handed circular polarization.

In order to understand the evolution of spin pumping (especially its sign) from the low-field regime ( $\left.H<H_{S F}\right)$ to the spin-flop regime $\left(H>H_{S F}\right)$, we resort to the following observations: $(i)$ At low fields, the chirality of spin precession (right-handed vs. left-handed) is locked to the spin angular 
momentum (spin-down vs. spin-up), see Figure 6.6. While the former refers to the rotational direction of the Néel vector, the latter refers to the z-component of the total magnetization. Therefore, we are able to tell the sign of spin pumping from either the Néel vector precession or the spin angular momentum. (ii) In the spin-flop regime, a finite magnetization along the magnetic field is induced in the ground state, which, when driven into resonance, always rotates in a righthanded manner similar to a ferromagnet [85]. Correspondingly, the non-equilibrium spin angular momentum of the SFM is always spin-down. Therefore, we can again tell the sign of spin pumping by simply focusing on the direction of spin angular momentum.

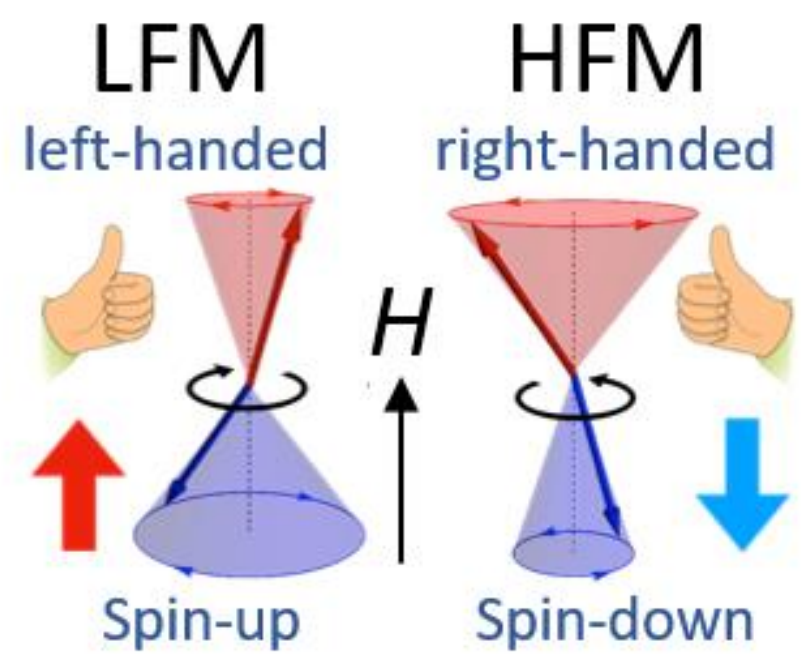

Figure 6.6: Illustration of the types of handed-ness of the precession of the Néel vector based on the precession of the sublattice magnetizations. The external field is applied along the anisotropy axis. 


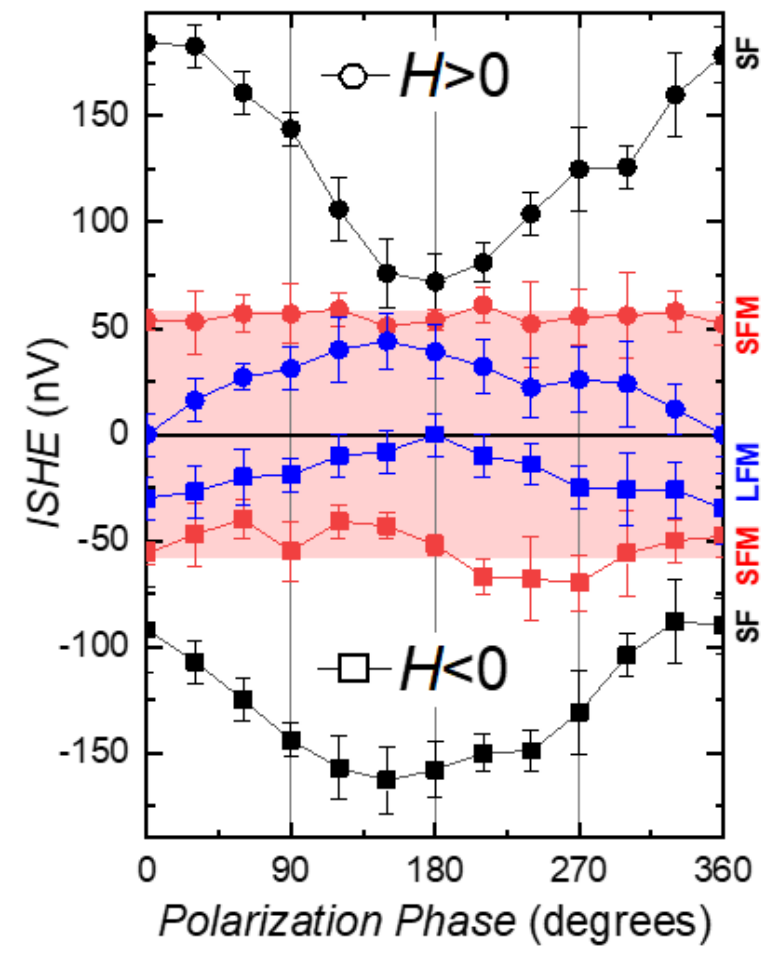

Figure 6.7: Magnitudes of the ISHE signals for the LFM, SF and SFM resonances as a function of polarization value for both positive and negative field.

As schematically displayed in Fig. 6.8, point 1 (HFM) and point 4 (SFM) clearly show opposite signs in their spin angular momenta, hence opposite pumped spin currents. This indicates that in the intermediate regime (the narrow window of spin-flop transition), there must be a point across which the total spin angular momentum flips sign. The HFM (SFM) characteristics dominates just below (above) that particular point, as marked by point 2 (point 3). However, the exact position of this critical point and how the eigenmodes evolve in the vicinity of that point can only be 
determined numerically in the presence of a finite misalignment angle. In Figure 5.13, we calculated the net equilibrium magnetization as a function of field, where we can qualitatively verify the above behavior. The subtle behavior in the vicinity of spin-flop transition calls for a much more rigorous measurement with more available microwave frequencies, which goes beyond the capacity and the scope of this thesis.

Interestingly, once deep into the SFM region, the ISHE signal no longer exhibits a dependence on the microwave polarization. Moreover, the sign of the observed ISHE at the SFM resonance is not consistent with the coherent picture discussed in the previous paragraph. This strongly suggests that spin currents from the SFM resonance are of thermal origin and are not related to coherent spin pumping at high fields. In fact, surface effects are likely to modify the magnetic anisotropy, slightly breaking its uniaxial symmetry. As a result, the Néel vector always flops into an easy direction perpendicular to the magnetic field, and correspondingly, the SFM mode becomes linearly-polarized and coexists with another linearly-polarized mode of a different frequency. We note that coherent spin pumping only stems from the uniform precession of the Néel vector (i.e., $k=0$ ). If the uniform precession becomes linearly polarized, there is simply no dc spin current. However, microwave heating reaches a maximum at the SFM resonance, creating a temperature gradient across the $\mathrm{MnF}_{2} / \mathrm{Pt}$ heterostructure that results in a spin Seebeck current flowing into the Pt. The spin Seebeck effect involves all magnon modes from both linearly-polarized branches throughout the Brillouin zone, where circular polarization is gradually retrieved as the wave vector $k$ goes larger. With the above considerations, we believe that the polarization-independent ISHE signal observed at the high-field SFM most likely originates from the spin Seebeck effect. 


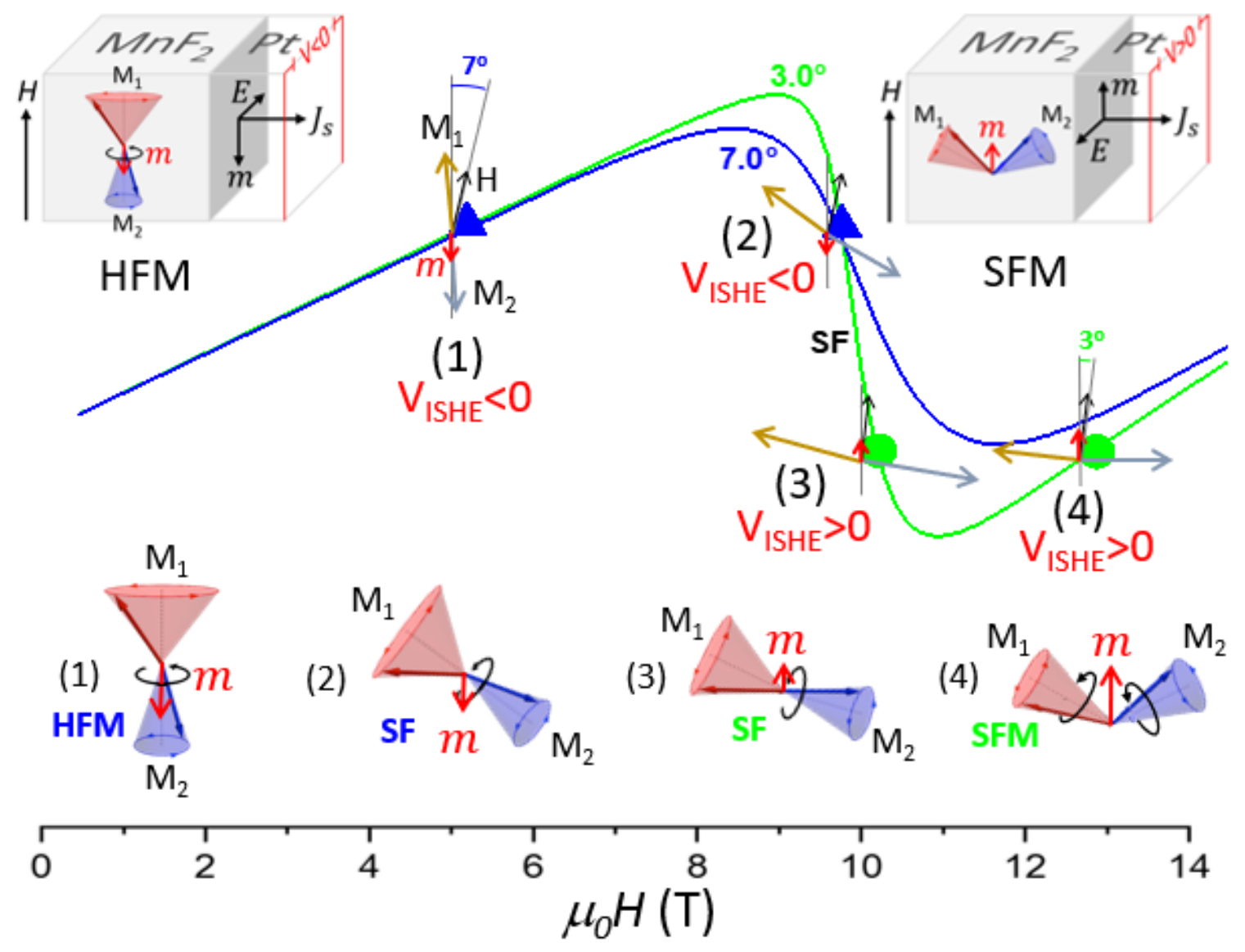

Figure 6.8: Illustration of the orientations of the sublattice magnetizations $\boldsymbol{M}_{1}$ and $\boldsymbol{M}_{2}$ and the applied field $\boldsymbol{H}$ with the anisotropy axis along the z-direction for four resonance points (1-2 for $395 \mathrm{GHz}$ and 3-4 for 240G $\mathrm{Gz}$ ) representative of the changes in AF dynamics while transitioning from the high frequency $A F$ resonance mode to the spin-flop mode through the spin-flop transition. The upper sketches represent the orientation and spin polarization of the pumped spin current and the induced ISHE electric field with respect to the measuring circuit in the sample. The lower insets illustrate the precessional cones of $\boldsymbol{M}_{1}$ and $\boldsymbol{M}_{2}$ for each resonance points discussed here. 


\section{CHAPTER 7 : CONCLUSIONS}

This thesis has successfully exhibited spin pumping from an antiferromagnetic insulator $\mathrm{MnF}_{2}$ for the first time. The nature of the signal convincingly proves the origin of the ISHE voltage signal is coherent dynamical spin pumping and not the incoherent spin Seebeck effect. Future work in this project can include study of temperature dependence of the ISHE signal as well as spin pumping in other systems. As suggested by Cheng and Brataas, $\mathrm{FeF}_{2}$ is the next promising candidate similar to $\mathrm{MnF}_{2}$ for spin pumping measurements and may exhibit interesting physics particularly close to the zero field degeneracy[46]. In addition, biaxial systems like $\mathrm{NiF}_{2}$ may exhibit inertial behavior and can work as THz oscillators as theoretically predicted by Slavin et.al. [86]. Thus, this thesis project can be advanced in many different directions.

The results presented in this thesis open door to advancements in controlling and understanding spin-transfer torques in antiferromagnetic-based systems. Future work in spin pumping may also reveal the relation between the structural symmetries of antiferromagnets, the characteristics of their spin dynamics and the polarization of the associated $\mathrm{THz}$ signals, which can help to design future generation of spintronics devices with antiferromagnets as the major component. 


\section{APPENDIX A: AFMR AND ISHE RESULTS FROM SAMPLES WITH}

$M_{2} F_{2}$ SUBSTRATE AND MNF 2 SUBSTRATE 
The project initiated with thin films of $\mathrm{MnF}_{2}$ grown on $\mathrm{MgF}_{2}$ substrate. Figure 1 illustrates the sample composition.

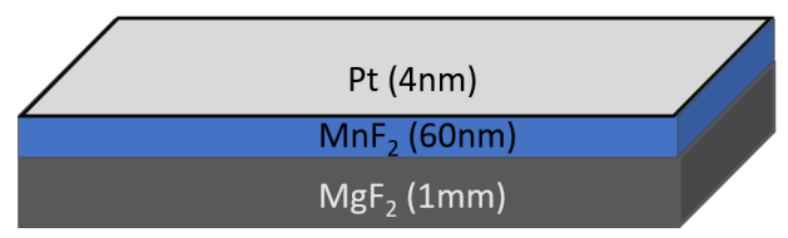

Figure 1: Sample composition of $\mathrm{MnF}_{2}$ on $\mathrm{MgF}_{2}$ substrate

The AFMR spectra of the sample revealed very broad features which made any resonance points indiscernible. The ISHE voltage measurement showed a signal that was again quite broad and asymmetrical as shown in Figure 2. The signal peak occurs at a point below the spin-flop transition, but individual signals associated to resonance points were not observed. The signal does reverse sign with field reversal but does not do so with reversal of polarization of microwaves. The nature of the signal is also unchanged as higher frequencies are sampled although the magnitude of the signal decreases as the frequency increases (Figure 3) which can be attributed to the fact that the power of the source decreases as one goes up from $120 \mathrm{GHz}$ to $336 \mathrm{GHz}$. 


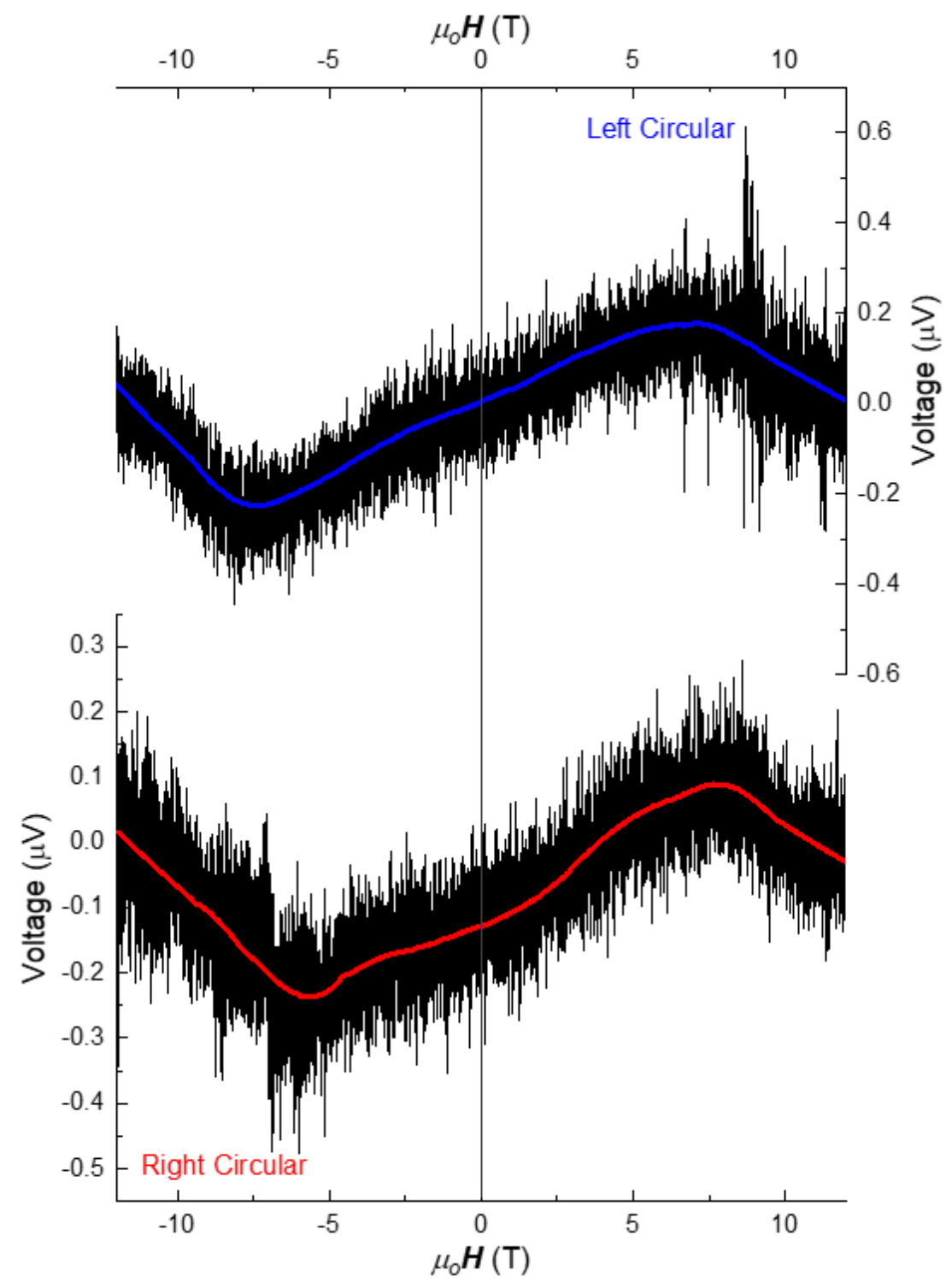

Figure 2: ISHE signal obtained from $\mathrm{MnF}_{2}$ grown atop $\mathrm{MgF}_{2}$ substrate for right handed and left handed circular polarization at $120 \mathrm{GHz}$. 


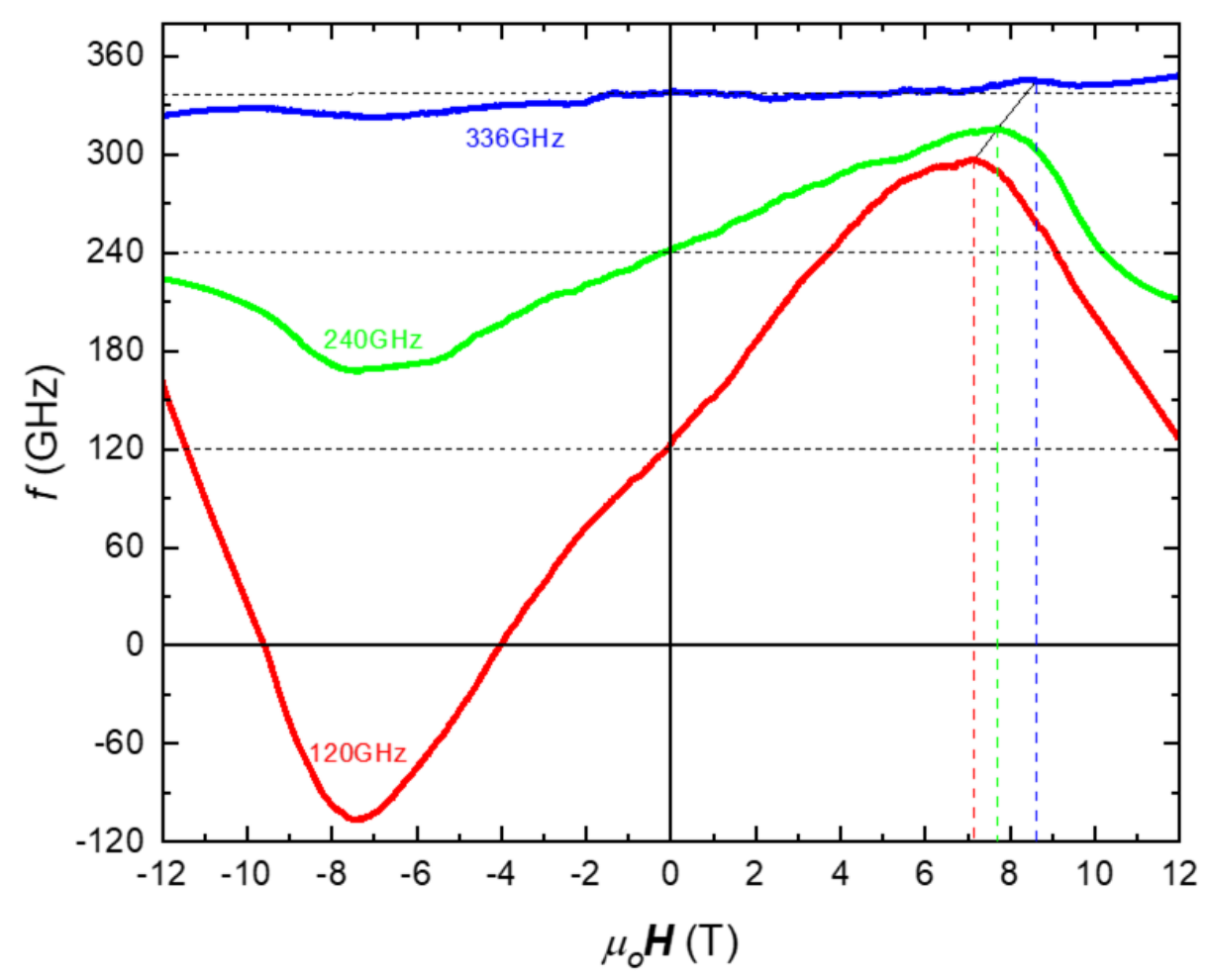

Figure 3: ISHE signal from $\mathrm{MnF}_{2}$ thin film (60nm) grown atop $M g F_{2}$ at $120 \mathrm{GHz}, 240 \mathrm{GHz}$ and $336 \mathrm{GHz}$ with right handed circular polarization

The failure to observe AFMR can be attributed to the fact that the AFMR films were very thin to absorb microwaves. The ISHE signals are also indistinguishable which leads to the possibility that the magnetization of $\mathrm{MnF}_{2}$ (antiferromagnetic) is not commensurate with that of $\mathrm{MgF}_{2}$ (which is diamagnetic in nature). This led to a change in methodology of sample growth. In order to ensure that proper AFMR is observed and the magnetization is uniform throughout the sample, single crystal $\mathrm{MnF}_{2}$ was chosen as substrate $(2 \mathrm{~mm})$. 


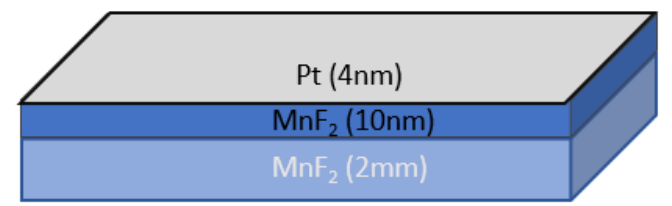

Figure 4: Sample composition of $\mathrm{MnF}_{2}$ on single crystal $\mathrm{MnF}_{2}$

The AFMR spectra obtained from the sample shown in Figure 5 was quite intense due to complete absorption of microwaves that made finding the exact position of resonance impossible. Figure 5 exhibits the AFMR spectra for low fields for both circular polarization. The spectra does show selective intensity towards the handed-ness of polarization. 


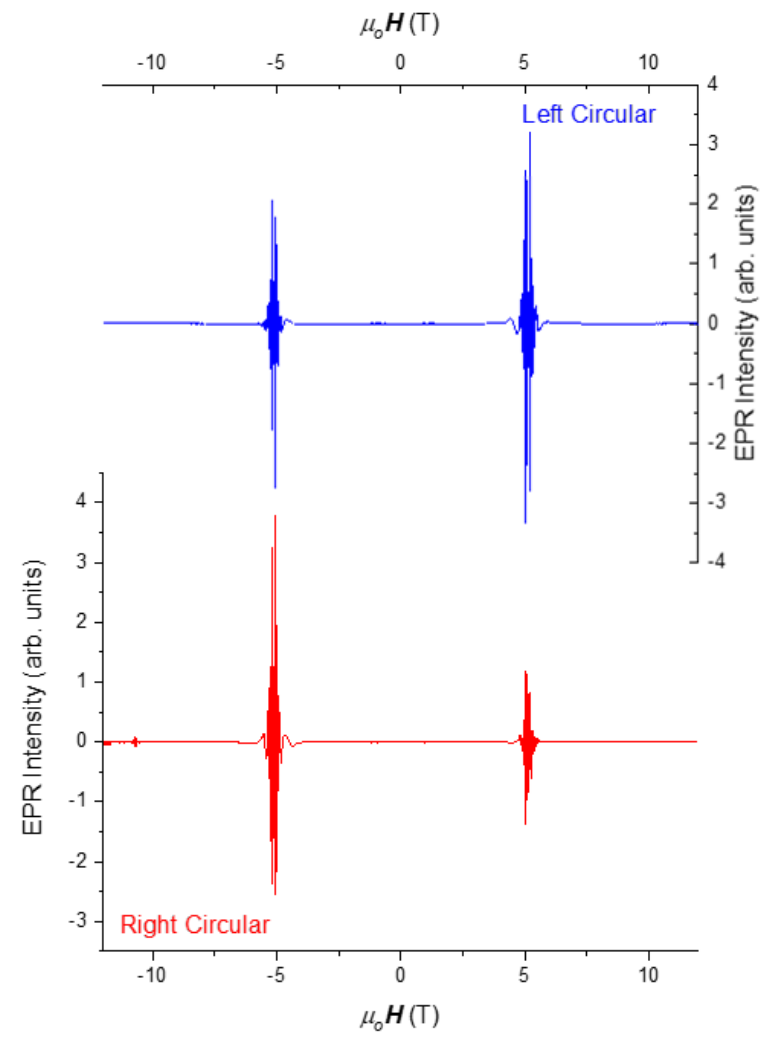

Figure 5: AFMR spectra of thin film $M n F_{2}$ grown on top of single crystal $\mathrm{MnF}_{2}(2 \mathrm{~mm})$ for left and right circular polarization.

The inability of obtaining discernible resonance signal for AFMR was attributed to the thickness of the $\mathrm{MnF}_{2}$ crystal. The spin pumping signal was also very noisy. As a result, another set of sample was prepared with the single crystal polished to a thickness of $200-300 \mu \mathrm{m}$. Even though an ideal thickness of $100 \mu \mathrm{m}$ was expected to give well resolved EPR signal, the thickness was chosen to be $200-300 \mu \mathrm{m}$ due to the fragility of the sample. Thus, a sample made of $200-300 \mu \mathrm{m}$ thick $\mathrm{MnF}_{2}$ single crystal was chosen as a substrate to grow $10 \mathrm{~nm}$ of $\mathrm{MnF}_{2}$ and then $4 \mathrm{~nm}$ of Pt. The thin film of $\mathrm{MnF}_{2}$ served to create a smooth surface for Pt to be deposited via e-beam 
evaporation so as to create a smooth interface between the antiferromagnet and the non-magnetic material. Such sample provided the results presented in this thesis. 
APPENDIX B: SPIN PUMPING RESULTS FOR 120GHZ 
The results of spin pumping at $120 \mathrm{GHz}$ needs to be discussed separately. $120 \mathrm{GHz}$ belongs to the low frequency AF resonance mode at low fields. The spin-flop transition and the spin-flop mode are quite close to each other and even a small misalignment can render these resonance points unobservable as seen from the AFMR spectra calculations in Figure 5.11. The resonance spectra for the three samples have already been discussed in Chapter 5. This appendix will include the spin pumping results at $120 \mathrm{GHz}$ obtained from Sample 2. The results from Sample 1 are similar to the ones in Sample 2.

Figure 7 illustrates the ISHE signal corresponding to the low-field AF resonance mode at $120 \mathrm{GHz}$ in Sample 2. Despite the asymmetric signal observed on the positive (negative) field for left (right) handed circular polarization, the signals behave in the same way as they do for $240 \mathrm{GHz}$. When compared with high frequency $395 \mathrm{GHz}$, the spin pumping picture explained in Figure 6.7 is still consistent. Though signal is observed at both positive and negative field, the intensity of signal is higher for either positive or negative field for a given polarization. Since the misalignment of this sample was larger, the spin flop transition and the spin flop mode are inaccessible and not observed. The ISHE signals at $120 \mathrm{GHz}$ have the aforementioned asymmetry that makes a quantitative analysis of the results difficult. 


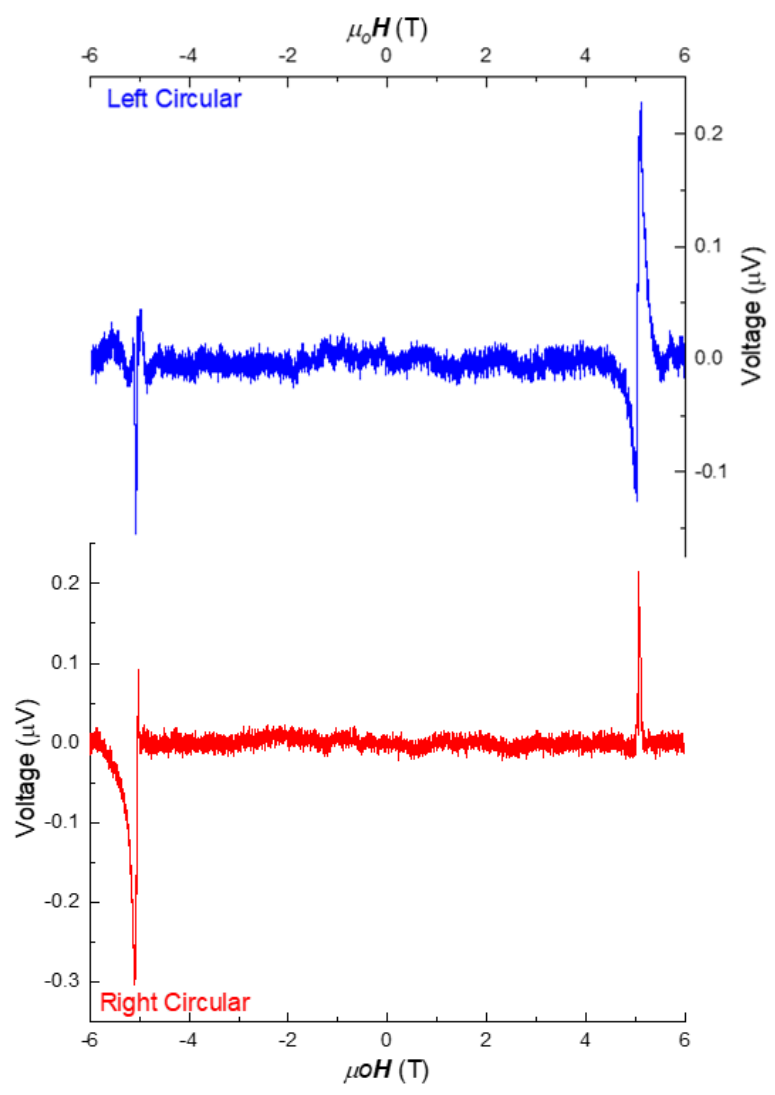

Figure 6: ISHE signal corresponding to the low-field AF resonance mode at $120 \mathrm{GHz}$ for left and right circular polarization in Sample 2.

The asymmetry may have its origins in an experimental artifact associated with this frequency. In addition to this, the high power of the frequency source $(150 \mathrm{~mW}$ which is highest out of the four frequencies) may have generated heating effect leading to a spin Seebeck signal being superimposed on the ISHE signal originating from spin pumping. This can be concluded from the fact that the amplitude of the signal $(\sim 250 \mathrm{nV})$ is many times larger than the ones from other frequencies. 

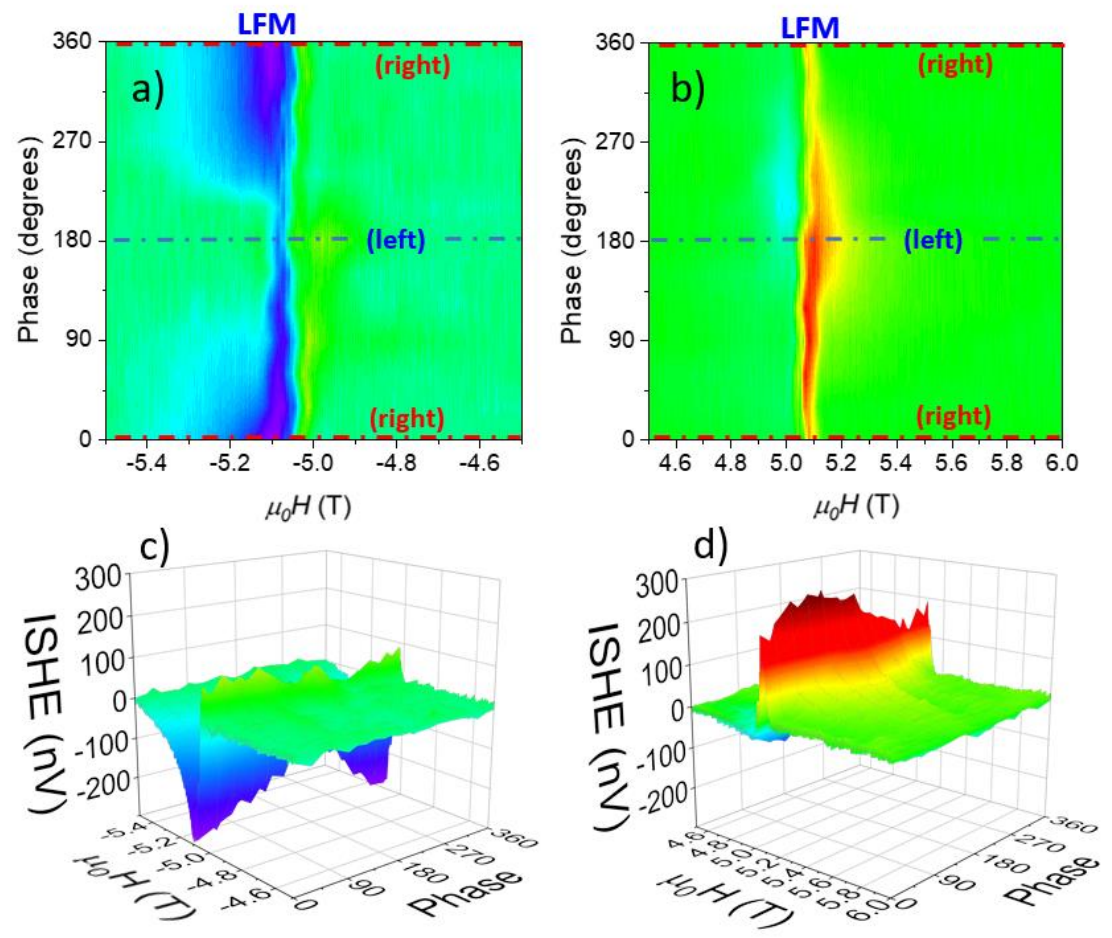

Figure 7: Polarization Modulation at 120GHz: Modulation of the ISHE signal at the LFM resonance in Sample 3 at 120GHz.

Figure 7 exhibits the results of polarization modulation of the ISHE signal at $120 \mathrm{GHz}$ and it can be observed that the polarization dependency follows the same pattern as for the higher frequencies: signal is more intense for left (right) handed circular polarization in the positive (negative) field. Thus, the fact that the overall behavior agrees with the observations at higher frequencies reinforces the interpretations provided in Chapter 6. 


\section{REFERENCES}

1. R. Duine, Spintronics: An alternating alternative. Nature materials, (2011). 10(5): p. 344.

2. J. Sinova, I. Žutić, New moves of the spintronics tango. Nature materials, (2012). 11(5): p. 368.

3. T. Kampfrath, A. Sell, G. Klatt, A. Pashkin, S. Mährlein, T. Dekorsy, M. Wolf, M. Fiebig, A. Leitenstorfer, R. Huber, Coherent terahertz control of antiferromagnetic spin waves. Nature Photonics, (2011). 5(1): p. 31.

4. J. Stohr, X-Rays Unravel the Puzzle of Exchange Bias.

5. L. Néel, Magnetism and local molecular field. Science, (1971). 174(4013): p. 985-992.

6. W.H. Meiklejohn, C.P. Bean, New magnetic anisotropy. Physical Review, (1957). 105(3): p. 904.

7. J. Nogués, I.K. Schuller, Exchange bias. Journal of Magnetism and Magnetic Materials, (1999). 192(2): p. 203-232.

8. W. Zhang, K.M. Krishnan, Epitaxial exchange-bias systems: From fundamentals to future spin-orbitronics. Materials Science and Engineering: R: Reports, (2016). 105: p. 120.

9. W. Zhang, M.B. Jungfleisch, W. Jiang, J.E. Pearson, A. Hoffmann, F. Freimuth, Y. Mokrousov, Spin Hall effects in metallic antiferromagnets. Physical review letters, (2014). 113(19): p. 196602.

10. W. Zhang, M.B. Jungfleisch, F. Freimuth, W. Jiang, J. Sklenar, J.E. Pearson, J.B. Ketterson, Y. Mokrousov, A. Hoffmann, All-electrical manipulation of magnetization dynamics in a ferromagnet by antiferromagnets with anisotropic spin Hall effects. Physical Review B, (2015). 92(14): p. 144405.

11. J. Mendes, R. Cunha, O.A. Santos, P. Ribeiro, F. Machado, R. Rodríguez-Suárez, A. Azevedo, S. Rezende, Large inverse spin Hall effect in the antiferromagnetic metal Ir 20 Mn 80. Physical Review B, (2014). 89(14): p. 140406.

12. Z. Qiu, J. Li, D. Hou, E. Arenholz, A.T. N'Diaye, A. Tan, K.-i. Uchida, K. Sato, S. Okamoto, Y. Tserkovnyak, Spin-current probe for phase transition in an insulator. Nature communications, (2016). 7: p. 12670.

13. L. Frangou, S. Oyarzun, S. Auffret, L. Vila, S. Gambarelli, V. Baltz, Enhanced spin pumping efficiency in antiferromagnetic IrMn thin films around the magnetic phase transition. Physical review letters, (2016). 116(7): p. 077203.

14. P. Merodio, A. Ghosh, C. Lemonias, E. Gautier, U. Ebels, M. Chshiev, H. Béa, V. Baltz, W. Bailey, Penetration depth and absorption mechanisms of spin currents in Ir 20Mn80 and Fe50Mn50 polycrystalline films by ferromagnetic resonance and spin pumping. Applied Physics Letters, (2014). 104(3): p. 032406.

15. Z. Wei, A. Sharma, A. Nunez, P. Haney, R. Duine, J. Bass, A.H. MacDonald, M. Tsoi, Changing exchange bias in spin valves with an electric current. Physical review letters, (2007). 98(11): p. 116603.

16. S.M. Wu, W. Zhang, K. Amit, P. Borisov, J.E. Pearson, J.S. Jiang, D. Lederman, A. Hoffmann, A. Bhattacharya, Antiferromagnetic spin Seebeck effect. Physical review letters, (2016). 116(9): p. 097204. 
17. S. Seki, T. Ideue, M. Kubota, Y. Kozuka, R. Takagi, M. Nakamura, Y. Kaneko, M. Kawasaki, Y. Tokura, Thermal generation of spin current in an antiferromagnet. Physical review letters, (2015). 115(26): p. 266601.

18. S. Rezende, R. Rodríguez-Suárez, A. Azevedo, Theory of the spin Seebeck effect in antiferromagnets. Physical Review B, (2016). 93(1): p. 014425.

19. I. Jacobs, Spin-flopping in MnF 2 by high magnetic fields. Journal of Applied Physics, (1961). 32(3): p. S61-S62.

20. I. Jacobs, P. Lawrence, Metamagnetic phase transitions and Hysteresis in FeCl 2. Physical Review, (1967). 164(2): p. 866.

21. M. Hagiwara, K. Katsumata, H. Yamaguchi, M. Tokunaga, I. Yamada, M. Gross, P. Goy, A Complete Frequency-Field Chart for the Antiferromagnetic Resonance in MnF 2. International journal of infrared and millimeter waves, (1999). 20(4): p. 617-622.

22. D. Kriegner, K. Výborný, K. Olejník, H. Reichlová, V. Novák, X. Marti, J. Gazquez, V. Saidl, P. Němec, V. Volobuev, Multiple-stable anisotropic magnetoresistance memory in antiferromagnetic MnTe. Nature communications, (2016). 7: p. 11623.

23. P. Wadley, B. Howells, J. Železný, C. Andrews, V. Hills, R.P. Campion, V. Novák, K. Olejník, F. Maccherozzi, S. Dhesi, Electrical switching of an antiferromagnet. Science, (2016). 351(6273): p. 587-590.

24. M. Hirschberger, S. Kushwaha, Z. Wang, Q. Gibson, S. Liang, C.A. Belvin, B.A. Bernevig, R.J. Cava, N.P. Ong, The chiral anomaly and thermopower of Weyl fermions in the half-Heusler GdPtBi. Nature materials, (2016). 15(11): p. 1161.

25. P. Tang, Q. Zhou, G. Xu, S.-C. Zhang, Dirac fermions in an antiferromagnetic semimetal. Nature Physics, (2016). 12(12): p. 1100.

26. L. Šmejkal, Y. Mokrousov, B. Yan, A.H. MacDonald, Topological antiferromagnetic spintronics. Nature Physics, (2018). 14(3): p. 242.

27. T. Higuchi, M. Kuwata-Gonokami, Control of antiferromagnetic domain distribution via polarization-dependent optical annealing. Nature communications, (2016). 7: p. 10720.

28. N. Duong, T. Satoh, M. Fiebig, Ultrafast manipulation of antiferromagnetism of NiO. Physical review letters, (2004). 93(11): p. 117402.

29. A. Kimel, B. Ivanov, R. Pisarev, P. Usachev, A. Kirilyuk, T. Rasing, Inertia-driven spin switching in antiferromagnets. Nature Physics, (2009). 5(10): p. 727.

30. J. Nishitani, T. Nagashima, M. Hangyo, Terahertz radiation from antiferromagnetic $\mathrm{MnO}$ excited by optical laser pulses. Applied Physics Letters, (2013). 103(8): p. 081907.

31. A. Kirilyuk, A.V. Kimel, T. Rasing, Ultrafast optical manipulation of magnetic order. Reviews of Modern Physics, (2010). 82(3): p. 2731.

32. T. Seifert, S. Jaiswal, U. Martens, J. Hannegan, L. Braun, P. Maldonado, F. Freimuth, A. Kronenberg, J. Henrizi, I. Radu, Efficient metallic spintronic emitters of ultrabroadband terahertz radiation. Nature photonics, (2016). 10(7): p. 483.

33. A.S. Núnez, R. Duine, P. Haney, A.H. MacDonald, Theory of spin torques and giant magnetoresistance in antiferromagnetic metals. Physical Review B, (2006). 73(21): p. 214426.

34. Y. Xu, S. Wang, K. Xia, Spin-transfer torques in antiferromagnetic metals from first principles. Physical review letters, (2008). 100(22): p. 226602. 
35. P. Haney, D. Waldron, R. Duine, A. Núñez, H. Guo, A.H. MacDonald, Ab initio giant magnetoresistance and current-induced torques in $\mathrm{Cr} / \mathrm{Aw} \mathrm{Cr}$ multilayers. Physical Review B, (2007). 75(17): p. 174428.

36. H.B.M. Saidaoui, A. Manchon, X. Waintal, Spin transfer torque in antiferromagnetic spin valves: From clean to disordered regimes. Physical Review B, (2014). 89(17): p. 174430.

37. H.B.M. Saidaoui, X. Waintal, A. Manchon, Robust spin transfer torque in antiferromagnetic tunnel junctions. Physical Review B, (2017). 95(13): p. 134424.

38. Z. Wei, J. Basset, A. Sharma, J. Bass, M. Tsoi, Spin-transfer interactions in exchangebiased spin valves. Journal of Applied Physics, (2009). 105(7): p. 07 D108.

39. M. Tsoi. Towards antiferromagnetic metal spintronics. in 2008 8th IEEE Conference on Nanotechnology. (2008). IEEE.

40. E. Gomonay, V. Loktev, Spintronics of antiferromagnetic systems. Low Temperature Physics, (2014). 40(1): p. 17-35.

41. H.V. Gomonay, R.V. Kunitsyn, V.M. Loktev, Symmetry and the macroscopic dynamics of antiferromagnetic materials in the presence of spin-polarized current. Physical Review B, (2012). 85(13): p. 134446.

42. H.V. Gomonay, V.M. Loktev, Spin transfer and current-induced switching in antiferromagnets. Physical Review B, (2010). 81(14): p. 144427.

43. R. Cheng, M.W. Daniels, J.-G. Zhu, D. Xiao, Ultrafast switching of antiferromagnets via spin-transfer torque. Physical Review B, (2015). 91(6): p. 064423.

44. P. Ross, M. Schreier, J. Lotze, H. Huebl, R. Gross, S.T. Goennenwein, Antiferromagentic resonance detected by direct current voltages in MnF2/Pt bilayers. Journal of Applied Physics, (2015). 118(23): p. 233907.

45. R. Cheng, J. Xiao, Q. Niu, A. Brataas, Spin pumping and spin-transfer torques in antiferromagnets. Physical review letters, (2014). 113(5): p. 057601.

46. Ø. Johansen, A. Brataas, Spin pumping and inverse spin Hall voltages from dynamical antiferromagnets. Physical Review B, (2017). 95(22): p. 220408.

47. J. Železný, H. Gao, K. Výborný, J. Zemen, J. Mašek, A. Manchon, J. Wunderlich, J. Sinova, T. Jungwirth, Relativistic Néel-order fields induced by electrical current in antiferromagnets. Physical review letters, (2014). 113(15): p. 157201.

48. K. Olejník, T. Seifert, Z. Kaspar, V. Novák, P. Wadley, R.P. Campion, M. Baumgartner, P. Gambardella, P. Nemec, J. Wunderlich, THz electrical writing speed in an antiferromagnetic memory. arXiv preprint arXiv:1711.08444, (2017).

49. H. Reichlová, D. Kriegner, V. Holý, K. Olejník, V. Novák, M. Yamada, K. Miura, S. Ogawa, H. Takahashi, T. Jungwirth, Current-induced torques in structures with ultrathin IrMn antiferromagnets. Physical Review B, (2015). 92(16): p. 165424.

50. R.A. Erickson, Neutron diffraction studies of antiferromagnetism in manganous fluoride and some isomorphous compounds. Physical Review, (1953). 90(5): p. 779.

51. C. Kittel, P. McEuen, P. McEuen, "Introduction to solid state physics". (Vol. 8. 1996: Wiley New York).

52. J.C. Slater, Cohesion in monovalent metals. Physical Review, (1930). 35(5): p. 509.

53. J.C. Slater, Atomic shielding constants. Physical Review, (1930). 36(1): p. 57. 
54. L. Landau, E. Lifshitz, On the theory of the dispersion of magnetic permeability in ferromagnetic bodies, in Perspectives in Theoretical Physics. (1992), Elsevier. p. 51-65.

55. S.V.e. Von Sovskii, "Ferromagnetic resonance: the phenomenon of resonant absorption of a high-frequency magnetic field in ferromagnetic substances". 2016: Elsevier).

56. F. Keffer, C. Kittel, Theory of antiferromagnetic resonance. Physical Review, (1952). 85(2): p. 329.

57. T. Nagamiya, Theory of Antiferromagnetism and Antiferromagnetic Resonance Absorption, II. Progress of Theoretical Physics, (1951). 6(3): p. 350-355.

58. T. Nagamiya, Theory of Antiferromagnetism and Antiferromagnetic Resonance Absorption, I. Progress of Theoretical Physics, (1951). 6(3): p. 342-349.

59. T. Nagamiya, K. Yosida, R. Kubo, Antiferromagnetism. Advances in Physics, (1955). 4(13): p. 1-112.

60. S. Foner, Antiferromagnetic and ferrimagnetic resonance, in Magnetism. (1963), Elsevier. p. 383-447.

61. T. Shinjo, "Nanomagnetism and spintronics". 2013: Elsevier).

62. P. Grünberg, R. Schreiber, Y. Pang, M. Brodsky, H. Sowers, Layered magnetic structures: Evidence for antiferromagnetic coupling of Fe layers across Cr interlayers. Physical review letters, (1986). 57(19): p. 2442.

63. M.N. Baibich, J.M. Broto, A. Fert, F.N. Van Dau, F. Petroff, P. Etienne, G. Creuzet, A. Friederich, J. Chazelas, Giant magnetoresistance of (001) Fe/(001) Cr magnetic superlattices. Physical review letters, (1988). 61(21): p. 2472.

64. W. Pauli, Über Gasentartung und Paramagnetismus. Zeitschrift für Physik A Hadrons and nuclei, (1927). 41(2-3): p. 81-102.

65. P.A.M. Dirac, The quantum theory of the electron. Proceedings of the Royal Society of London. Series A, Containing Papers of a Mathematical and Physical Character, (1928). 117(778): p. 610-624.

66. J. Sinova, S.O. Valenzuela, J. Wunderlich, C. Back, T. Jungwirth, Spin hall effects. Reviews of Modern Physics, (2015). 87(4): p. 1213.

67. M. Dyakonov, Spin Hall Effect. arXiv preprint arXiv:1210.3200, (2012).

68. Y. Tserkovnyak, A. Brataas, G.E. Bauer, Spin pumping and magnetization dynamics in metallic multilayers. Physical Review B, (2002). 66(22): p. 224403.

69. Y. Tserkovnyak, A. Brataas, G.E. Bauer, Enhanced Gilbert damping in thin ferromagnetic films. Physical review letters, (2002). 88(11): p. 117601.

70. E. Saitoh, M. Ueda, H. Miyajima, G. Tatara, Conversion of spin current into charge current at room temperature: Inverse spin-Hall effect. Applied physics letters, (2006). 88(18): p. 182509.

71. O. Mosendz, V. Vlaminck, J. Pearson, F. Fradin, G. Bauer, S. Bader, A. Hoffmann, Detection and quantification of inverse spin Hall effect from spin pumping in permalloy/normal metal bilayers. Physical Review B, (2010). 82(21): p. 214403.

72. S. Takahashi, Physical Principles of Spin Pumping. Handbook of Spintronics, (2016): p. 1445-1480.

73. K. Uchida, S. Takahashi, K. Harii, J. Ieda, W. Koshibae, K. Ando, S. Maekawa, E. Saitoh, Observation of the spin Seebeck effect. Nature, (2008). 455(7214): p. 778. 
74. K. Uchida, J. Xiao, H. Adachi, J.-i. Ohe, S. Takahashi, J. Ieda, T. Ota, Y. Kajiwara, H. Umezawa, H. Kawai, Spin seebeck insulator. Nature materials, (2010). 9(11): p. 894.

75. C. Jaworski, J. Yang, S. Mack, D. Awschalom, J. Heremans, R. Myers, Observation of the spin-Seebeck effect in a ferromagnetic semiconductor. Nature materials, (2010). 9(11): p. 898.

76. A. Kirihara, K.-i. Uchida, Y. Kajiwara, M. Ishida, Y. Nakamura, T. Manako, E. Saitoh, S. Yorozu, Spin-current-driven thermoelectric coating. Nature materials, (2012). 11(8): p. 686.

77. Y. Kajiwara, K. Harii, S. Takahashi, J.-i. Ohe, K. Uchida, M. Mizuguchi, H. Umezawa, H. Kawai, K. Ando, K. Takanashi, Transmission of electrical signals by spin-wave interconversion in a magnetic insulator. Nature, (2010). 464(7286): p. 262.

78. Z. Wang, Y. Sun, M. Wu, V. Tiberkevich, A. Slavin, Control of spin waves in a thin film ferromagnetic insulator through interfacial spin scattering. Physical review letters, (2011). 107(14): p. 146602.

79. J. Holanda, D. Maior, O. Alves Santos, L. Vilela-Leão, J. Mendes, A. Azevedo, R. Rodríguez-Suárez, S. Rezende, Spin Seebeck effect in the antiferromagnet nickel oxide at room temperature. Applied Physics Letters, (2017). 111(17): p. 172405.

80. J. Li, Z. Shi, V.H. Ortiz, M. Aldosary, C. Chen, V. Aji, P. Wei, J. Shi, Spin Seebeck Effect from Antiferromagnetic Magnons and Critical Spin Fluctuations in Epitaxial FeF 2 Films. Physical Review Letters, (2019). 122(21): p. 217204.

81. J. Van Tol, L.-C. Brunel, R. Wylde, A quasioptical transient electron spin resonance spectrometer operating at 120 and $240 \mathrm{GHz}$. Review of scientific instruments, (2005). 76(7): p. 074101.

82. W. Lin, C. Chien, Evidence of pure spin current. arXiv preprint arXiv:1804.01392, (2018).

83. R. Cheng, D. Xiao, A. Brataas, Terahertz antiferromagnetic spin Hall nano-oscillator. Physical Review Letters, (2016). 116(20): p. 207603.

84. Y. Wang, C. Song, B. Cui, G. Wang, F. Zeng, F. Pan, Room-temperature perpendicular exchange coupling and tunneling anisotropic magnetoresistance in an antiferromagnetbased tunnel junction. Physical review letters, (2012). 109(13): p. 137201.

85. C. Kittel, Theory of antiferromagnetic resonance. Physical Review, (1951). 82(4): p. 565.

86. R. Khymyn, I. Lisenkov, V. Tiberkevich, B.A. Ivanov, A. Slavin, Antiferromagnetic THz-frequency Josephson-like oscillator driven by spin current. Scientific reports, (2017). 7: p. 43705. 